\title{
SYMPTOM-RELATED ATTRIBUTIONAL BIASES IN SCHIZOPHRENIA AND BIPOLAR DISORDER
}

\author{
by \\ NICOLE ALEXANDRA SANFORD \\ B.A., McGill University, 2010
}

A THESIS SUBMITTED IN PARTIAL FULFILLMENT OF THE REQUIREMENTS FOR THE DEGREE OF

\author{
MASTER OF SCIENCE \\ in \\ THE FACULTY OF GRADUATE AND POSTDOCTORAL STUDIES \\ (Neuroscience)
}

THE UNIVERSITY OF BRITISH COLUMBIA

(Vancouver)

April 2014

(C) Nicole Alexandra Sanford, 2014 


\begin{abstract}
Impairments in social cognition are known to have severe impacts on functional outcome in schizophrenia. Attributional biases (i.e., biases towards assigning blame/credit to oneself, other people, or to the situation) are of particular interest due to their direct relevance to paranoid delusions and potential implication in cognitive-based treatments. A "defense" model of paranoid delusions proposed by Bentall, Kinderman, and Kaney (1994) suggests that paranoid individuals possess an extreme self-serving bias, with a specific tendency to blame other people as opposed to the situation (i.e., personalizing bias). In addition, an inability to integrate belief-disconfirming information is thought to underlie the fixedness of delusions. However, these biases have not been investigated simultaneously to check for additive or multiplicative effects on associations with symptoms. Moreover, previous studies have failed to take into account the heterogeneity of the symptoms of psychosis. The present research employed structural equation modelling and constrained principal component analysis in schizophrenia patients, bipolar disorder patients, and healthy individuals to examine the extent to which group differences and symptom severity could predict patterns of responding on a novel attributional bias task, designed to assess an individual's ability to integrate contextual information in conjunction with attributional reasoning. In line with the defense model of paranoia, it was predicted that schizophrenia patients with severe paranoid delusions would display enhanced self-serving and personalizing biases. However, no differences between diagnostic- or symptom-based participant groups were found. Conversely, the severity of symptoms of overactive disorganization in schizophrenia and bipolar disorder patients predicted higher situation attributions and self-blame (specifically when such attributions were unsupported by the available evidence), while higher depression in healthy participants was negatively related to situation attributions and lower self-credit. These
\end{abstract}


findings suggest that non-self-serving bases may be non-specifically related to high psychopathology, while an ability to integrate socially-relevant contextual information to consider other people's roles in events may be reduced in overactive disorganization in mental illness, and is negatively related to depression in healthy individuals. The absence of noticeable group differences in attributional biases illustrates the importance of employing a multivariate symptom-based approach when studying complex cognitive processes in psychosis. 


\section{Preface}

Dr. Heungsun Hwang (Department of Psychology, McGill University) provided the GSCA t-test results comparing the FIT values reported in Study 1; otherwise, all studies reported are based on my analyses of data collected by Dr. Todd Woodward's research group. None of the work reported has been previously published.

This research was approved by the University of British Columbia Clinical Research Ethics Board (certificate number H07-01442). 


\section{Table of Contents}

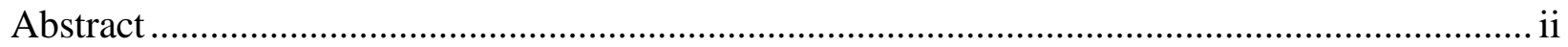

Preface

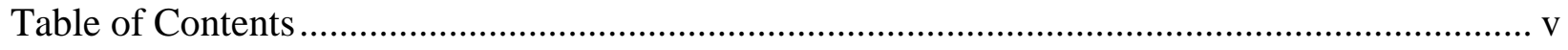

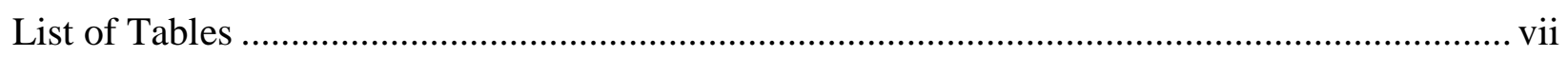

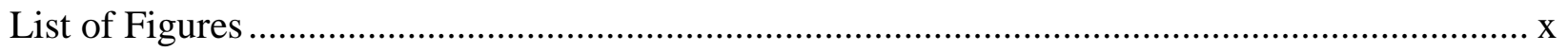

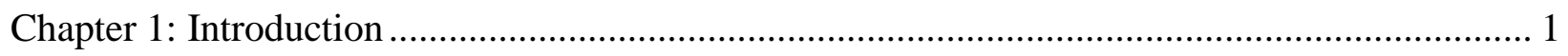

1.1. Overview ...................................................................................... 1

1.2. Social Cognition in Schizophrenia ........................................................ 2

1.3. Attributional Bias .................................................................................. 4

1.4. Bias Against Disconfirmatory Evidence .............................................. 9

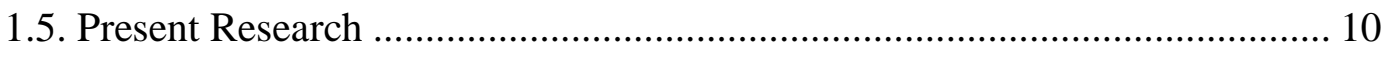

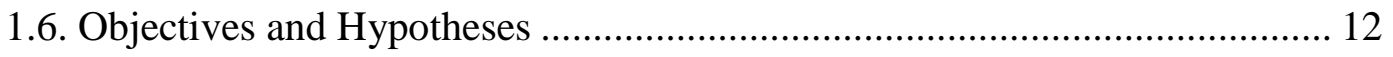

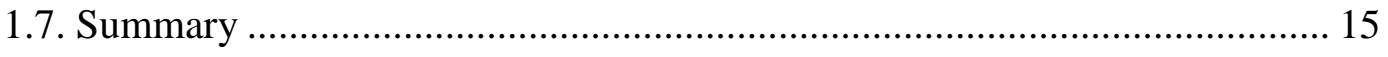

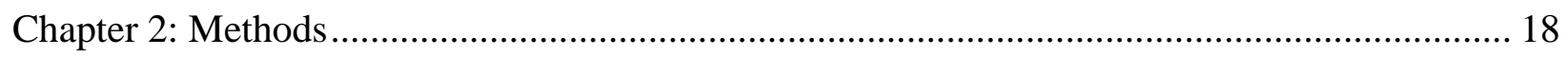

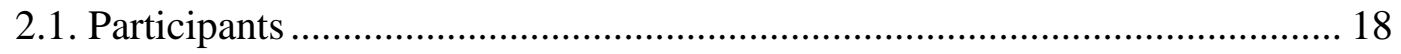

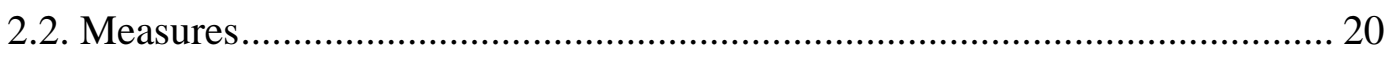

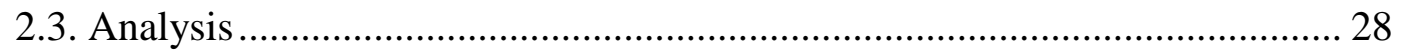

Chapter 3: Attributional Biases on the ASB Task (Study 1) ............................................... 35

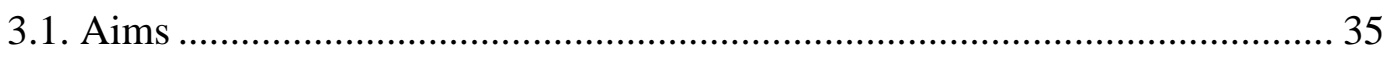

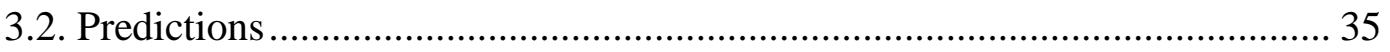

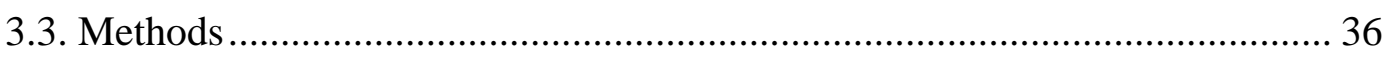

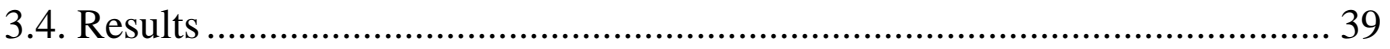




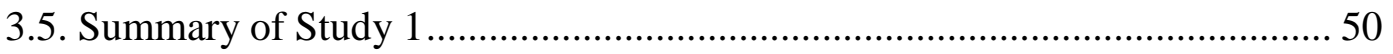

Chapter 4: Symptom-Related Attributional Biases (Study 2) ................................................... 52

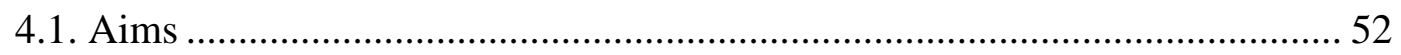

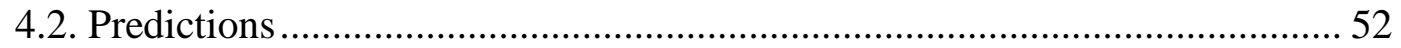

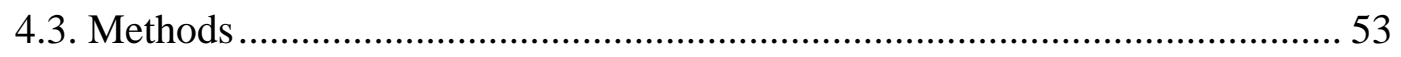

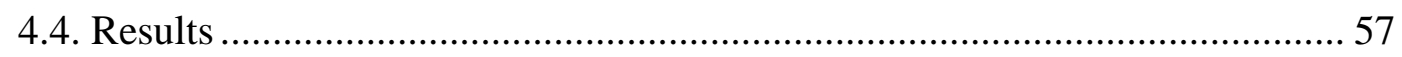

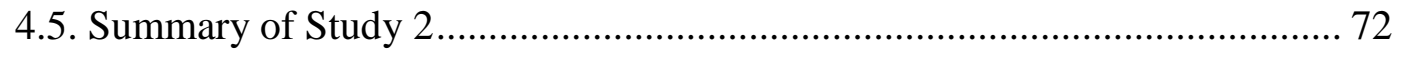

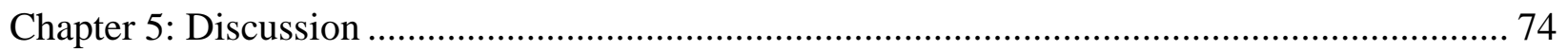

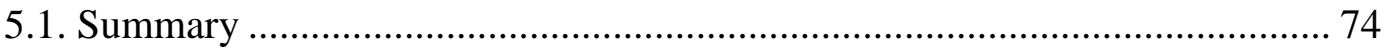

5.2. Cognitive Operations of the ASB Task ............................................................. 76

5.3. Group Comparisons................................................................................... 79

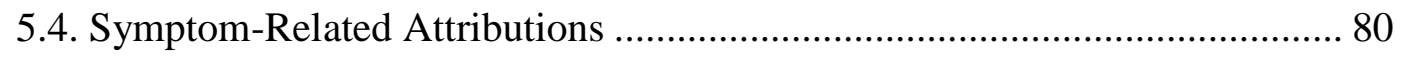

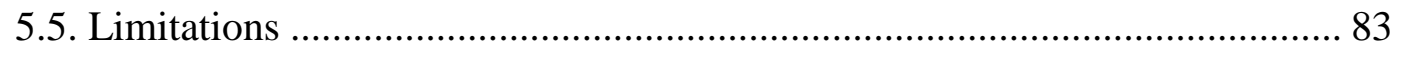

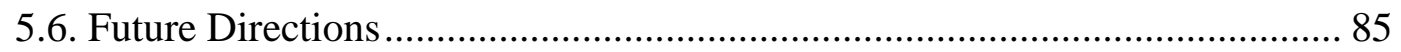

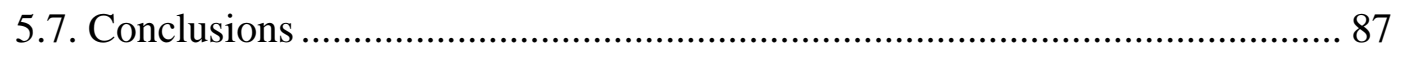

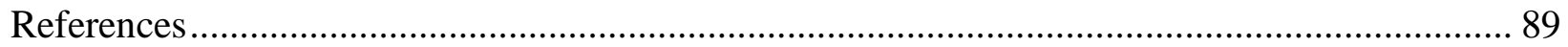

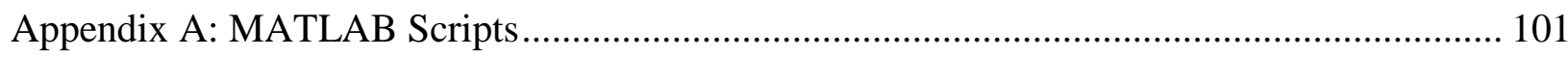

Eigenvalues and Scree Plot (Example from Study 2) ........................................ 101

Constrained Principal Component Analysis (Example from Study 2) ............... 102

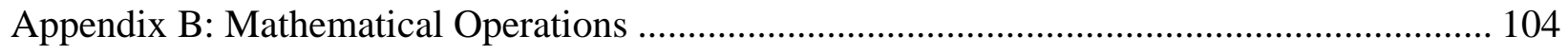

Principal Component Analysis (PCA) ................................................................ 104

Constrained Principal Component Analysis (CPCA) ………………………..... 105

Generalized Structured Component Analysis (GSCA) ....................................... 106 


\section{List of Tables}

Table 3.1. Study 1 sample means and standard deviations (unless otherwise specified) for demographic variables and IQ.

Table 3.2. Study 1 mean SSPI symptom scores for schizophrenia patients and bipolar disorder patients (standard deviations in parentheses)

Table 3.3. Mean attribution ratings for healthy, schizophrenia, and bipolar disorder participant groups. Standard deviations in parentheses.

Table 3.4. Component loadings for the ASB task, with four components extracted from PCA. . 43

Table 3.5. Component loadings for each latent variable and each group from unconstrained multi-group GSCA. 46

Table 3.6. Component loadings for each latent variable from constrained multi-group GSCA. . 47

Table 4.1. Study 2 sample means and standard deviations (unless otherwise specified) for demographic variables and IQ.

Table 4.2. Study 2 SSPI symptom scores for all patients $(n=95)$. 55

Table 4.3. Distribution of variance for CPCA no. 1 in patients $(Z=$ initial attribution ratings, $G=$ SSPI). 57

Table 4.4. GC component loadings and $G$ loadings from CPCA no. 1 in patients $(Z=$ initial attribution ratings, $G=\mathrm{SSPI})$. 58

Table 4.5. $E$ component loadings from CPCA no. 1 in patients $(Z=$ initial attribution ratings, $G=$

SSPI). 58

Table 4.6. Distribution of variance for CPCA no. 2 in patients $(Z=$ all observed attribution ratings, $G=\mathrm{SSPI})$ 
Table 4.7. $G C$ component loadings and $G$ loadings from CPCA no. 2 in patients $(Z=$ all observed attribution ratings, $G=\mathrm{SSPI})$.

Table 4.8. $E$ component loadings from CPCA no. 2 in patients $(Z=$ all observed attribution ratings, $G=\mathrm{SSPI})$. 61

Table 4.9. Distribution of variance for CPCA no. 3 in patients $(Z=$ all attribution ratings with neurocognitive measures partialled out, $G=\mathrm{SSPI})$

Table 4.10. $G C$ component loadings and $G$ loadings from CPCA no. 3 in patients $(Z=$ all attribution ratings with neurocognitive measures partialled out, $G=\mathrm{SSPI}$ ).

Table 4.11. $E$ component loadings from CPCA no. 3 in patients $(Z=$ all attribution ratings with neurocognitive measures partialled out, $G=\mathrm{SSPI})$

Table 4.12. Distribution of variance for CPCA no. 1 in nonclinical participants $(Z=$ initial attribution ratings, $G=\mathrm{CAPE})$

Table 4.13. $G C$ component loadings and $G$ loadings from CPCA no. 1 in nonclinical participants ( $Z=$ initial attribution ratings, $G=\mathrm{CAPE})$. 66

Table 4.14. E component loadings from CPCA no. 1 in nonclinical participants $(Z=$ initial attribution ratings, $G=\mathrm{CAPE})$ 66

Table 4.15. Distribution of variance for CPCA no. 2 in nonclinical participants $(Z=$ all observed attribution ratings, $G=\mathrm{CAPE})$

Table 4.16. $G C$ component loadings and $G$ loadings from CPCA no. 2 in nonclinical participants $(Z=$ all observed attribution ratings, $G=\mathrm{CAPE})$. 68

Table 4.17. $E$ component loadings from CPCA no. 2 in nonclinical participants $(Z=$ all observed attribution ratings, $G=\mathrm{CAPE})$ 
Table 4.18. Distribution of variance for CPCA no. 3 in nonclinical participants $(Z=$ all attribution ratings with neurocognitive measures partialled out, $G=\mathrm{CAPE})$................ 70

Table 4.19. GC component loadings and $G$ loadings from CPCA no. 3 in nonclinical participants $(Z=$ all attribution ratings with neurocognitive measures partialled out, $G=\mathrm{CAPE}) \ldots . .71$

Table 4.20. $E$ component loadings from CPCA no. 3 in nonclinical participants $(Z=$ all attribution ratings with neurocognitive measures partialled out, $G=\mathrm{CAPE}) \ldots \ldots \ldots \ldots \ldots \ldots . . . . . .72$ 


\section{List of Figures}

Figure 2.1. Two items from the CAPE questionnaire ....................................................... 23

Figure 2.2. Screenshot of a trial from the ASB task after the first statement has been presented.27

Figure 2.3. Screenshot of a trial from the ASB task after the second statement has been

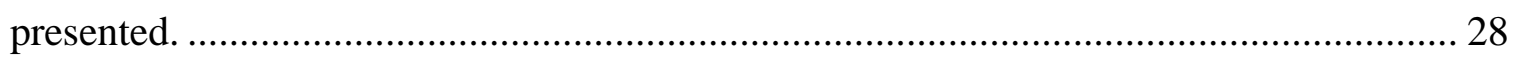

Figure 3.1. Mean initial attribution ratings for positive and negative scenarios (whole sample, $n=$

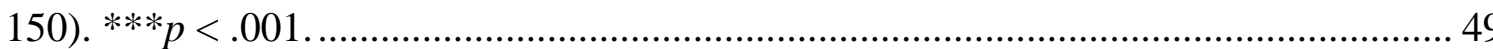




\section{Chapter 1: Introduction}

\subsection{Overview}

The complexity of symptoms in psychotic illness presents an intriguing area for cognitive research, as symptoms are often accompanied by dysfunctional thinking and reasoning processes known as cognitive biases. Thus, a major goal of cognitive neuropsychiatry research is to uncover the cognitive biases that play a role in the formation and/or maintenance of psychotic symptoms, which may be achieved by developing cognitive tasks that correlate with the severity of symptoms of interest. Much of our understanding of behaviour in schizophrenia, for example, stems from the development of tasks that engage the cognitive processes associated with given symptoms of psychosis (for reviews, see Bell, Halligan, \& Ellis, 2006; Blackwood, Howard, Bentall, \& Murray, 2001; Colbert, Peters, \& Garety, 2010; Garety \& Freeman, 1999; Moritz et al., 2010; Woodward, Moritz, Menon, \& Klinge, 2008).

The development of symptom-based cognitive measures is of considerable interest because of their potential clinical applications for psychological interventions in schizophrenia; for example, research on a treatment known as metacognitive training (MCT; Moritz, Veckenstedt, Randjbar, Vitzthum, \& Woodward, 2011) suggests that helping delusional patients

learn to recognize and counter the cognitive processes characterizing their illness without discussing personal beliefs can reduce the impact of delusions, defined as fixed false beliefs that (1) are maintained despite counter-evidence and rational counter-argument, (2) would be dismissed by members of the same social-cultural environment, and (3) are held with great conviction (American Psychiatric Association, 2000). Although antipsychotic medication is standard treatment for schizophrenia, such conjunctive psychological interventions - based on appropriate symptom-based cognitive operations - are necessary due to the limited effectiveness 
and compliance in pharmacological treatments (Byerly, Nakonezny, \& Lescouflair, 2007; Leucht, Arbter, Engel, Kissling, \& Davis, 2009). In addition, the development of reliable cognitive tasks can help guide neuroimaging research by allowing investigators to elucidate the underlying neurological activity associated with a given cognitive operation, and how this may be altered in mental illness (e.g., Seidel et al., 2012; Speechley, Woodward, \& Ngan, 2013).

Significant advances have been achieved in cognition research in schizophrenia. Delusions have been of particular interest due to their potential amenability to nonpharmacological treatments such as MCT (Moritz et al., 2011) and their connections with several known reasoning biases. For example, a data-gathering bias ("jumping to conclusions"), observed on probabilistic reasoning tasks, is thought to illustrate the hasty belief formation giving rise to and helping to maintain delusions (Sanford, Lecomte, Leclerc, Wykes, \& Woodward, 2013; Speechley, Whitman, \& Woodward, 2010; Woodward, Munz, Leclerc, \& Lecomte, 2009). Related to this is the bias against belief-disconfirming evidence, which is thought to underlie the fixedness of delusions (Riccaboni et al., 2012; Sanford, Veckenstedt, Moritz, Balzan, \& Woodward, 2014; Speechley, Ngan, Moritz, \& Woodward, 2012; Woodward, Moritz, Cuttler, \& Whitman, 2006). Biases in causal attributions (e.g., extreme self-serving biases) have been implicated in psychosis as well (Combs \& Penn, 2008), and may reflect a special case of jumping to conclusions (Merrin, Kinderman, \& Bentall, 2007). Such attributional biases are important to understand, given the substantial impact of social cognitive functioning on functional outcome and general well-being in schizophrenia.

\subsection{Social Cognition in Schizophrenia}

Understanding and utilizing social cues and behaviours relies on a knowledge base and skill set known as "social cognition". A consensus meeting sponsored by the National Institute of 
Mental Health (NIMH; Green et al., 2008) recognized five domains of social cognition: (1) theory of mind, or mentalizing (i.e., ability to infer intentions, dispositions, and beliefs of others), (2) social perception (i.e., ability to identify social roles, societal rules, and social context), (3) social knowledge (i.e., awareness of the roles, rules, and goals that characterize social situations and guide social interactions), (4) attributional bias (i.e., tendency to assign responsibility to oneself, another person, or the situation for a given event), and (5) emotion processing and perception (i.e., identifying, facilitating, understanding, and managing emotions). All domains of social cognition are known to be impaired in schizophrenia (Savla, Vella, Armstrong, Penn, \& Twamley, 2013), independent of neurocognition (Mehta et al., 2013). Further, impaired social cognition uniquely predicts functional outcome in schizophrenia (Couture, Penn, \& Roberts, 2006; Fett et al., 2011). In fact, accumulating evidence for the importance of social cognition in functional outcome has sparked an interest in the development of social cognitive treatments for psychosis (Fiszdon \& Reddy, 2012; Horan, Kern, Green, \& Penn, 2008; Kurtz \& Richardson, 2012; Marsh, Langdon, Harris, \& Coltheart, 2013). Moreover, abnormalities in neurological activity have been demonstrated in areas of the brain associated with social cognition in schizophrenia patients during social cognitive tasks (Das, Lagopoulos, Coulston, Henderson, \& Malhi, 2012; Mukherjee et al., 2014; Park et al., 2009; Walter et al., 2009), suggesting that some fundamental impairment in processing social stimuli is present in psychotic illness (BrunetGouet \& Decety, 2006). This is consistent with the "social brain" hypothesis of schizophrenia, which posits that psychosis has persisted as a costly trade-off of the evolution of complex social cognition (Burns, 2006).

Among the social cognitive domains listed above, attributional bias is of particular interest because of its direct relevance to delusions, as misattributions of events and others' 
actions may play a vital role in maintaining a delusional belief. For example, it is clearly observable in the clinical setting that patients with paranoid delusions misperceive threat in the actions of others, and it has been proposed that this arises from an extreme self-serving bias, with a particular tendency to blame other people as opposed to the situation for negative events (Bentall et al., 1994). In addition, attributional biases can be readily targeted in cognitive-based treatments (Moritz, Woodward, \& Burlon, 2005); therefore, a greater understanding of the nature of these biases and how they are maintained is warranted so that social-cognitive and behavioural treatment methods can be developed and refined.

\subsection{Attributional Bias}

\subsubsection{Measuring attributional style}

An attributional bias is a marked preference for assigning responsibility to oneself, other people, or the situation for given events, which may vary depending on whether the scenario in question is positive (e.g., "you won a prize") or negative (e.g., "you failed a test"). This has typically been measured using the Attributional Style Questionnaire (ASQ; Peterson et al., 1982) and/or the Internal, Personal and Situational Attributions Questionnaire (IPSAQ; Kinderman \& Bentall, 1996a). Although other forms of attribution measures exist (e.g., perceived hostility of others' intentions, typically assessed with the Ambiguous Intentions Hostility Questionnaire; Combs, Penn, Wicher, \& Waldheter, 2007), the following overview will be limited to the ASQ and IPSAQ, as these specifically measure explicit causal attributions, the primary area of interest in the present research (and the most applicable to attribution-focused cognitive training).

The ASQ was originally developed to study attributional style in depression (Peterson et al., 1982). In this task, a positive or negative self-referent event is presented (e.g., "you get a raise"), and the participant is asked to write down a possible cause of this event and to rate this 
cause on three dimensions: (1) internality (the degree to which this cause is due to oneself or to external sources), (2) stability (likelihood of this cause being present in the future), and (3) globalness (degree to which this cause may influence a wide range of events in addition to the specific scenario presented). Although widely used in attributional studies, the ASQ has been criticized for the low reliability of the internality dimension (Reivich, 1995; Tennen \& Herzenberger, 1985), which is centrally important in measuring attributional bias. Further, the internality dimension does not make a distinction between external-personal (i.e., another person/group of people) and external-situational attributions (i.e., circumstances/chance), but rather is unidimensional and labeled with the anchors "totally due to me" and "totally due to other people or circumstances"; this can be problematic, as individuals often have difficulty categorizing attributions in this manner (White, 1991).

To address this limitation of the internality dimension, Kinderman and Bentall (1996a) designed the IPSAQ for use in their studies on persecutory delusions. This task is based on the ASQ, but makes a distinction between external-personal and external-situational attributions. After providing a possible cause for the scenario, the participant indicates whether this is most related to oneself, another person, or the situation (or, in a revised form, participants may assign a percentage value for each attribution; Moritz et al., 2010). From this, two measures of attributional bias are typically derived: the externalizing bias (EB) and the personalizing bias (PB). The EB score is normally computed by subtracting the number of "self" attributions for negative scenarios from the number of "self" attributions for positive scenarios, producing an index of a self-serving bias. It should be noted, however, that attributions for positive and negative events do not necessarily correlate, and it has been suggested that positive and negative 
attributions should be treated separately (Byrne \& MacLeod, 1997). The PB score is the proportion of external-personal attributions in all external attributions for negative events.

Although normal attributional biases exist in healthy individuals, extreme or dysfunctional attributional biases are thought to be tied to certain psychopathological conditions. For example, a self-serving bias (i.e., bias towards blaming external causes for negative events and/or attributing self-responsibility for positive events) appears to be a normal cognitive bias observed in healthy individuals, and is thought to help maintain a high level of self-esteem (Campbell \& Sedikides, 1999; Mezulis, Abramson, Hyde, \& Hankin, 2004). It has been proposed that paranoia arises from an extreme self-serving bias, with a particular bias towards blaming other people as opposed to the situation for negative events (Bentall et al., 1994). By contrast, a depressive attributional bias (based on Abramson and colleagues' learned helplessness theory; Abramson, Seligman, \& Teasdale, 1978) is characterized as a high degree of self-blame for negative events and/or external attributions for positive events (Kinderman \& Bentall, 1997). Attributional style in bipolar disorder has not been as well-studied, though some research suggests that an extreme attributional style (whether positive or negative) in bipolar patients may predict relapse of manic/hypomanic episodes and longer time to recover during depressive episodes (Stange, Sylvia, da Silva Magalhães, Frank, et al., 2013; Stange, Sylvia, da Silva Magalhães, Miklowitz, et al., 2013). Attributional style in psychosis has been studied extensively, but primarily with respect to paranoia.

\subsubsection{Psychosis and attributional style}

Interest in the role of attributional biases in the development of psychotic symptoms was sparked by Bentall and colleagues' pioneering work on attributions underlying persecutory delusions (Kaney \& Bentall, 1989). In a review of cognitive abnormalities associated with 
paranoia, Bentall et al. (1994) first proposed a "defense" account of paranoid delusions based on Higgins' self-discrepancy theory (Higgins, 1987), suggesting that these patients possess extreme self-serving attributional biases to defend against threats to self-esteem, which are activated by perceived discrepancies between their "actual" selves and "ideal" selves. These authors proposed that, in paranoid patients, minimizing these perceived discrepancies is achieved by formulating large discrepancies between self-perceptions and the self as thought to be perceived by others, and by extension perceive negative intentions of others, leading to persecutory ideation. The defense model was supported primarily by two studies. First, in a study of self-discrepancies in paranoid and depressed patients, Kinderman and Bentall (1996b) found that, although paranoid patients demonstrated high consistency between self-perceptions and their reported "ideal" selves, they displayed high discrepancies between self-perceptions and their selves as thought to be (negatively) perceived by their parents. Second, Kinderman and Bentall (1997) demonstrated that individuals with paranoid delusions showed a higher externalizing bias on the IPSAQ than depressed patients (with healthy controls scoring intermediately between the two patient groups), as well as a greater personalizing bias than both the depressed group and healthy controls; that is, when forming attributions for negative events, the paranoid group not only had a high tendency to externalize, but were more likely to assign blame to other people rather than the situation. Kinderman and Bentall (1997) proposed that, together, these findings suggested that patients with paranoid delusions have an extreme self-serving bias with a specific tendency to blame other people for negative events, which arises from a threat to one's self-esteem and leads to persecutory ideation. However, subsequent research on attributional biases underlying paranoid delusions has been inconsistent. 
Although some evidence supports a defensive attributional style underlying paranoid delusions (Diez-Alegría, Vázquez, Nieto-Moreno, Valiente, \& Fuentenebro, 2006), it may not be specific to acutely paranoid patients (Martin \& Penn, 2002). Most early studies on paranoid delusions and attributional style employed a between-groups design in which patients with paranoid delusions were compared with depressed patients and healthy controls (Kaney \& Bentall, 1989; Kinderman \& Bentall, 1997; Lyon, Kaney, \& Bentall, 1994). Depressed patients were often included as psychiatric controls because it was assumed that patients with paranoid delusions possess an underlying depressive psychopathology, and, indeed, paranoid patients typically showed a relative self-serving bias compared to patients with major depression. However, findings were less consistent when schizophrenia patients with paranoid delusions were compared to those without paranoid delusions (Sharp, Fear, \& Healy, 1997; Silverman \& Peterson, 1993). Moreover, the self-serving bias present in paranoid patients was often comparable to that observed in healthy individuals (Donohoe et al., 2012; Kinderman \& Bentall, 1997). In studies using the IPSAQ, some researchers demonstrated a more specific association with the personalizing bias measure than with a general self-serving bias (Combs et al., 2009; Diez-Alegría et al., 2006; Kinderman \& Bentall, 1997; Langdon, Ward, \& Coltheart, 2010), although others suggested that personalizing may be related to general severity of delusions rather than paranoia in particular (Fear, Sharp, \& Healhy, 1996; Martin \& Penn, 2002). Randjbar, Veckenstedt, Vitzthum, Hottenrott, and Moritz (2011) proposed that the attributional style observed in paranoid patients may be better explained by a non-specific reduced sense of selfcausation, as per their observation that paranoid schizophrenia patients made fewer internal attributions than non-paranoid schizophrenia patients, patients with obsessive-compulsive disorder (OCD), and healthy individuals, regardless of whether the attribution was for a positive 
or negative event. They also found a significant positive correlation between positive symptoms and a "lack of control" bias (i.e., tendency to externalize, whether personal or situational, for both positive and negative scenarios), although this was specifically driven by a correlation with unusual thought content.

Research in attributional bias has yet to examine the fixedness of an individual's attributions in the face of conflicting information; that is, there has been extensive investigation of belief formation in the context of attributional reasoning, but not belief maintenance. Recall that a bias against belief-disconfirming evidence is thought to underlie the fixedness of delusions (Riccaboni et al., 2012; Sanford et al., 2014; Speechley et al., 2012; Woodward, Moritz, \& Chen, 2006; Woodward, Moritz, Cuttler, et al., 2006); it is unknown, however, whether a more specific association with paranoia would be present with respect to specific attributional beliefs.

\subsection{Bias Against Disconfirmatory Evidence}

A defining characteristic of delusions is that they are maintained with conviction despite counter-evidence and rational counter-argument. Previous research has demonstrated that a cognitive bias against disconfirmatory evidence (BADE) in schizophrenia is observable on delusion-neutral material, illustrating a cognitive process that may underlie the fixed aspect of delusions. A BADE is typically assessed in a cognitive task in which a participant rates the subjective plausibility of several interpretations of a given scenario, and may adjust these ratings based on accumulating evidence. For example, the statement, "Jenny can't fall asleep," may be interpreted in several ways (e.g., "Jenny is worried about her exam the next day," or "Jenny is

excited about Christmas morning"); as more statements are presented (e.g., "Jenny can't wait until it is finally morning"), it becomes increasingly apparent as to which interpretation is true. An unwillingness to down-rate initially plausible interpretations ("lures") that are subsequently 
revealed to be implausible is often present in schizophrenia (Moritz \& Woodward, 2006; Woodward et al., 2008) and particularly enhanced in delusional patients (Riccaboni et al., 2012; Woodward, Moritz, \& Chen, 2006; Woodward, Moritz, Cuttler, et al., 2006). A BADE has also been associated with delusion-proneness and schizotypy in healthy individuals (Buchy, Woodward, \& Liotti, 2007; Woodward, Buchy, Moritz, \& Liotti, 2007).

Factor analyses of the BADE task have revealed two underlying cognitive operations, reflecting (1) the degree to which disambiguating evidence is integrated ("evidence integration") and (2) unwillingness to rate high when justified ("conservatism"). Delusional schizophrenia patients appear to be specifically impaired in evidence integration, which may explain the apparent bias against disconfirmatory evidence observed on this task (Sanford et al., 2014; Speechley et al., 2012). Moreover, this BADE measure appears to be independent from other cognitive domains, including executive function (Woodward et al., 2007).

\subsection{Present Research}

The present research investigated attributional biases in the context of belief inflexibility in psychosis. Extreme attributional biases and an enhanced bias against belief-disconfirming evidence have been observed in schizophrenia patients, but have not been examined simultaneously in order to check for additive or multiplicative effects on associations with delusions. Given the considerable interest in paranoid delusions in attributional biases, as well as the role of a general BADE in the fixedness of delusions, it is pertinent to extend this area of research to investigation of a potential BADE effect in the context of attributional reasoning.

Traditional measures of attributional style such as the ASQ and IPSAQ have been useful for examining individuals' explicit attributional assumptions when presented with ambiguous events. As noted, however, this does not gauge an individual's willingness to revise their 
judgements when provided with more contextual information. The Attributional Style BADE (ASB) task, a variation of the BADE task developed by Dr. Todd Woodward's team, assesses attributional beliefs and their amenability to change by incorporating the IPSAQ into the traditional BADE paradigm. Like the IPSAQ, a positive or negative scenario is presented, and the participant evaluates the degree to which themselves, other people, or the situation are responsible. The ASB task features three independent, continuous rating scales with which the participant indicates his/her endorsement of each attributional locus. Further, the task provides the participant with the opportunity to adjust these ratings following additional information that insinuates a particular cause. For example, an individual may be presented with the scenario, "Your team won an important game"; first, the individual rates on three separate scales the responsibility of themselves, other people, and the situation. The individual is then presented with information about a possible cause (e.g., "Now imagine that the other team played poorly.") and may adjust his/her ratings accordingly, thereby providing a means to assess belief revision in the context of attributional reasoning. Thus, the ASB task allowed for the investigation of attributional beliefs and belief maintenance in the present research.

Previous research on attributional style in psychosis has been highly hypothesis-driven, focusing almost exclusively on paranoid delusions. However, a considerable amount of information may be lost when a priori assumptions lead to a focus on individual symptoms in the context of heterogeneous symptomatology. An alternative approach is to first isolate the variance of the attributional bias measure that is predictable from all symptoms on a given clinical scale, allowing a wide range of symptoms to contribute to the analysis, and for the hypothesized symptom to emerge as dominant if justified by the data. The cognitive processes that are directly predictable from psychopathology can then be determined, as well as which of these symptoms 
in particular have the highest correlations with these cognitive processes. Although some studies have sought to examine symptoms other than paranoia (e.g., Lincoln, Mehl, Kesting, \& Rief, 2011, who investigated attributional biases connected to negative symptoms in schizophrenia), there is an important distinction between the methods employed previously and the approach employed in the present research; that is, while previous studies have attempted to find relationships between standard attributional style measures (e.g., the EB score on the IPSAQ) and individual symptom measures, the present research investigated attribution-related cognitive operations, measured by task performance, that are predictable from all symptoms of psychosis. The advantage of this is that it does not restrict analyses to individual symptoms, but instead focuses on the investigation of cognitive processes that are directly predictable from psychopathology. Given the heterogeneity of psychosis, such a comprehensive approach may be more appropriate.

Taken together, the specific objectives of this research were to (1) determine the cognitive operations engaged in the ASB task, (2) compare attributional biases in healthy individuals, schizophrenia patients, bipolar disorder patients, and symptom-based patient subgroups, and (3) examine components of the ASB task that are directly predictable from psychopathology and schizotypical traits.

\subsection{Objectives and Hypotheses}

\subsubsection{Objective no. 1: Cognitive operations in the ASB task}

One objective of the present research was to investigate the cognitive processes engaged in the ASB task, and in particular, to determine whether a BADE could be observed in the context of attributional reasoning. This was achieved using principal component analysis (PCA) to determine the component structure of the ASB task. Although this is a novel measure of 
attributional style and of belief revision, similarities between the ASB and the traditional BADE tasks allow some general speculations to be made. Recall that previous component analyses of the BADE task identified two cognitive operations, reflecting (1) the degree to which disambiguating evidence is integrated ("evidence integration") and (2) unwillingness to rate high when justified ("conservatism"). The evidence integration component is of particular interest because it illustrates individual differences in BADE-like responding (i.e., being less inclined to revise plausibility ratings following evidence that disconfirms one's initial beliefs and points to an alternative interpretation). In general terms, an attribution-specific "evidence integration" component might be manifested in negative correlations between ratings for disconfirmed attributions and ratings for the "true" source of responsibility. For example, given the known tendency for both healthy and mentally ill individuals to display a self-serving bias, a selfserving "BADE" component could reflect a pattern of providing relatively low "self" attribution ratings and high external (other-person or situation) attribution ratings for scenarios involving a negative outcome, specifically when self-responsibility is implied (e.g., "You are rear-ended", followed by "Now imagine that you were talking on your cell phone while driving"). How such cognitive operations may tie into the component structure of the ASB task is described in further detail in the following chapter. This objective is addressed in Study 1 (Chapter 3).

\subsubsection{Objective no. 2: Group-based attributional biases}

The second objective of this research was to determine the extent to which differences between participant groups (healthy individuals, schizophrenia patients, bipolar disorder patients, and symptom-based patient subgroups) can predict responses on the ASB task, and to examine attributional biases that underlie potential group differences in responding. Although no specific predictions were made regarding differences between schizophrenia patients, bipolar disorder 
patients, and healthy individuals, differences between symptom-based patient subgroups (based on severity of paranoid delusions or depression) were expected. In line with the "defense" account of paranoia, it was predicted that schizophrenia patients with severe paranoid delusions would display an enhanced self-serving and personalizing bias compared with non-paranoid schizophrenia patients, bipolar disorder patients, and healthy individuals. Further, it was predicted that paranoid schizophrenia patients would be particularly resistant to changing such beliefs after being presented with disconfirming contextual information. Finally, it was predicted that patients with severe depression would display an attenuated self-serving bias before contextual information about a given scenario was presented; however, depressed patients were not expected to display a BADE. Again, the potential reflection of such biases in principal components is described in the following chapter. This objective is addressed in Study 1 (Chapter 3).

\subsubsection{Objective no. 3: Symptom-based attributional biases}

As previously noted, a large degree of heterogeneity exists in the symptomatology of psychotic illness. A promising alternative to studying differences between diagnostic categories is to examine symptoms that cross nosological boundaries (Blom \& Van Praag, 2011; Murray, 2011). Therefore, the final objective of the present research was to use a symptom-based, exploratory approach to investigate the components of the ASB task that are predictable from symptoms of psychosis and schizotypical traits, in a manner which allowed all symptoms to potentially contribute to relationships between attributional biases and psychopathology.

Again, in line with the defense model, paranoid delusions (and potentially other positive symptoms) were expected to correlate with a self-serving bias and personalizing bias. Further, a self-serving/personalizing-BADE was expected to correlate specifically with paranoid delusions; 
that is, although other symptoms may be associated with attributional biases, only delusions (and paranoia in particular) were expected to predict an unwillingness to revise such beliefs after receiving disconfirming information. Conversely, self-blame was expected to correlate with depression (and other negative symptoms, as negative symptoms in schizophrenia have been associated with an internalizing bias for negative events; Lincoln et al., 2011); however, such symptoms were not expected to predict an unwillingness to revise one's attribution ratings.

Psychotic symptoms (including paranoia) can manifest as subclinical, schizotypical traits in healthy individuals (Tabak \& Weisman de Mamani, 2013). This presents an opportunity to verify whether cognitive dysfunction is detectable in subclinical psychopathology, or only manifests with symptoms of clinical severity. Therefore, this same objective included an investigation of subclinical symptom dimensions in healthy individuals. Although the symptom scale employed in these nonclinical participants yields broader dimensional measures of symptoms (i.e., positive, negative, and depression), some tentative predictions were made: in general terms, the positive symptom dimension was expected to correlate with a self-serving bias, and the depression and negative symptom dimensions were expected to negatively correlate with such a bias. Objective no. 3 was addressed in patients and nonclinical participants in Study 2 (Chapter 4).

\subsection{Summary}

The development of cognitive tasks that correlate with symptoms of psychosis is crucial for understanding the biased cognitive operations that play a role in the formation and/or maintenance of such symptoms. Notably, social cognition uniquely predicts functional outcome in schizophrenia (Couture et al., 2006; Fett et al., 2011), signifying an important domain for clinical research. Though all domains of social cognition are known to be impaired in 
schizophrenia (Savla et al., 2013), attributional bias is of particular interest because of the direct involvement of misattributions in delusions, and paranoia in particular. Moreover, a bias against disconfirmatory evidence (BADE) is associated with delusions in schizophrenia, and may therefore specifically underlie the fixed aspect of delusions.

Attributional biases and BADE, although well-studied in psychosis, have not been investigated together. That is, current attributional bias tasks provide measures of initial attributional belief formation, which could be extended to evaluate the maintenance of attributional beliefs in the face of disconfirming evidence; this would allow the investigation of potential additive or multiplicative effects on associations between attributional biases and symptoms. The BADE task offers a useful paradigm to draw from, as its use in schizophrenia research has demonstrated impaired evidence integration in delusional patients. Therefore, the ASB task, which is a consolidation of the IPSAQ and BADE paradigms, could prove to be a particularly informative measure of attributional reasoning. The present research aimed to investigate this while taking into account the heterogeneity of psychosis, as the existing research on attributional biases in psychosis has focused almost exclusively on paranoia, and is lacking in exploratory, multivariate approaches.

The present research is divided into two studies, each of which follows a general approach of investigating the cognitive operations engaged in the ASB task, and which cognitive operations are predictable from the independent variable of interest. The results are presented in two chapters: Study 1 begins with an investigation of the overall component structure of the ASB task and the generalizability of this structure across participant groups (healthy individuals, schizophrenia patients, and bipolar disorder patients), and then investigates the extent to which diagnostic- and symptom-based group differences explain responding on the ASB task. Study 2 
examines components of the ASB task that underlie relationships between attributions and psychotic symptoms in schizophrenia and bipolar disorder patients, as well as relationships between attributions and schizotypical traits in nonclinical participants. The following chapter outlines the primary methods and statistical approaches that were employed in this research. The findings are summarized and discussed in the concluding chapter. 


\section{Chapter 2: Methods}

The present research used a cross-sectional design to examine performance on the Attributional Style Bias Against Disconfirmatory Evidence (ASB) task in healthy individuals, bipolar disorder patients, schizophrenia patients, and symptom-based patient subgroups, as well as to explore relationships between attributional biases and symptoms of psychosis (or schizotypical traits, in healthy participants). This chapter presents the methods that were employed in this research, including: (1) participant characteristics and recruitment procedures, (2) cognitive/symptom measures and task administration, and (3) the primary statistical methods employed.

\subsection{Participants}

All analyses in this research were drawn from a dataset of 171 participants (49 schizophrenia patients, 51 bipolar disorder patients, and 71 healthy individuals). Patients with bipolar disorder served as a psychiatric comparison group, as this population is similar to schizophrenia patients in cognitive function and genetic and environmental susceptibility factors (Barrett et al., 2009). The majority of patients were chronically ill; only four (two schizophrenia patients and two bipolar disorder patients) were experiencing a first or early psychiatric episode.

Participants were recruited by advertisements or word-of-mouth, or, in many cases, patients were referred to the study by their mental health care providers. All participants provided written informed consent after a complete description of the study. Inclusion criteria for all participants were: (1) 19-55 years of age, and (2) fluent in English (having used English daily for more than 5 years). For Study 1 (Chapter 3), participants were excluded from the analysis if they had a known history of a neurological condition (e.g., stroke, epilepsy, encephalitis, etc.) or head injury resulting in concussion or loss of consciousness, or low intelligence (estimated IQ < 
70). In addition to these exclusion criteria, patients did not qualify if they (1) did not have a diagnosis of schizophrenia, psychosis, schizoaffective disorder, schizophreniform disorder or bipolar disorder, (2) had psychosis that was a direct consequence of substance abuse (e.g., cocaine, crystal methamphetamine, heroin, etc.), or (3) had a secondary or concurrent psychiatric diagnosis (e.g., major depressive disorder), although these criteria were somewhat relaxed for Study 2 (Chapter 4), which employed a correlational analysis between attributional biases and symptoms. Healthy controls were excluded from Study 1 if they (1) used hard drugs such as cocaine, crystal methamphetamine or heroin on a regular basis, or (2) had previously experienced or were currently experiencing any psychiatric condition or psychosis [though exceptions were made for Study 2 (Chapter 4), which investigated schizotypical traits in healthy participants, and so a possible personal or family history of psychiatric illness was accepted]. All diagnoses (including screening for the healthy control group) were confirmed by trained research staff using the Mini International Neuropsychiatric Interview (MINI; Sheehan et al., 1998), a thorough but brief structured clinical interview. Modules were included for psychotic disorders, mood disorders (major depressive episode, dysthymia, and manic/hypomanic episode), obsessive-compulsive disorder, generalized anxiety disorder, panic disorder, agoraphobia, social anxiety disorder, posttraumatic stress disorder, alcohol abuse/dependence, and non-alcohol psychoactive substance use disorders.

In addition to completing the experimental attributional style task, all participants underwent some form of thorough symptom assessment and standard neuropsychological testing. The specific measures of interest are described below. 


\subsection{Measures}

\subsubsection{Neurocognitive measures}

\section{Kaufmann Brief Intelligence Test}

The Kaufmann Brief Intelligence Test, $2^{\text {nd }}$ edition, (KBIT-II; Kaufman, 1990) was administered to assess general neurocognitive function. The KBIT-II provides a verbal IQ score, a non-verbal IQ score, and a general estimated IQ score that is a composite of the two. These measures were obtained to examine the degree to which performance on the attributional style task could be accounted for by general neurocognitive functioning, and to what extent symptoms could explain performance on the ASB task independent of general cognitive demands.

The verbal score is derived from two tests, one of which assesses verbal knowledge using a picture-based vocabulary test, and the other using riddles. The vocabulary portion consists of 60 items of increasing difficulty, which assess vocabulary and general knowledge about the world. For each item, the participant is shown an array of six illustrations or photographs, and the examiner says a word or asks a question of general knowledge. The participant is asked to respond by pointing to the picture that depicts the meaning of the word or the answer to the question. The riddles portion consists of 48 items of increasing difficulty that measure verbal comprehension, reasoning, and vocabulary knowledge. Similar to the vocabulary test, the participant is asked a riddle (e.g., "what has whiskers, is a common household pet, and meows?"), and then responds by either pointing to a picture that shows the answer to the riddle or by providing a single word that answers the riddle.

The non-verbal IQ score is derived from a matrix-reasoning test. This test consists of 46 items involving a series of meaningful (i.e., people or objects) or abstract (i.e., designs and symbols) visual stimuli. The participant is asked to decipher a relationship or rule among a set of 
pictures or patterns, and identify (from multiple choices) the picture or pattern that best completes or fits with the set of pictures/patterns based on this relationship/rule.

\section{Wisconsin Card-Sorting Test}

A computerized version of the Wisconsin Card-Sorting Test (WCST; Loong, 1991) was administered to assess executive functioning (specifically, response perseveration). This task, originally developed by Grant and Berg (1948), has been used extensively in neurocognitive testing to assess cognitive flexibility. In brief, the participant is instructed to sort a series of cards and to decide to which of four piles of cards the dealt card belongs. The cards can be sorted according to one of three possible criteria: (1) the geometric shape displayed on the card (stars, crosses, triangles, or circles), (2) the number of these shapes displayed $(1,2,3$, or 4$)$, or (3) the colour of the shapes displayed (red, yellow, green, or blue). The participant is not explicitly told what the sorting rule is, but they receive feedback as to whether their choice was correct or incorrect. However, the sorting criterion changes periodically, after the participant has learned the current rule. In the present research, the particular measure of interest was the percentage of perseverative errors made by the participant (i.e., errors in which the participant continues to sort according to the most recent rule learned instead of the new one). This score was included in the reported analyses to determine the extent to which an unwillingness to change one's attribution ratings could be due to cognitive bias after accounting for general response perseveration.

\subsubsection{Symptom assessment}

\section{Signs and Symptoms of Psychotic Illness}

Psychotic symptoms in schizophrenia and bipolar disorder patients were assessed with the Signs and Symptoms of Psychotic Illness scale (SSPI; Liddle, Ngan, Duffield, \& Warren, 2002). The SSPI is a semi-structured interview consisting of 20 symptoms, each rated on a 5- 
point scale (0-4, with $0=$ absent, $4=$ severe), with additional subscales for delusions (guilt/worthlessness, grandiosity, paranoia, and Schneiderian themes) and hallucinations (second person auditory and Schneiderian). To complete the assessment, the examiner asks the patient about his/her experiences and how s/he has been feeling over the past week, with questions directed to evaluate symptoms of psychosis.

\section{Community Assessment of Psychic Experiences}

Psychotic symptom proneness was assessed in healthy participants using the Community Assessment of Psychic Experiences (CAPE; Konings, Bak, Hanssen, van Os, \& Krabbendam, 2006). The CAPE is a self-report questionnaire, which consists of 42 questions that ask how often the participant has encountered particular experiences. This scale was designed to capture healthy manifestations of three dimensions of psychosis: positive symptoms (particularly reality distortion; i.e., hallucinations and delusions), negative symptoms, and depressive symptoms. 20 questions address instances of positive symptoms (e.g., "Do you ever hear voices when you are alone?"), 14 questions address negative symptoms (e.g., "Do you ever feel that you experience few or no emotions at important events?"), and 8 address symptoms of depression (e.g., "Do you ever cry about nothing?"). If the participant endorses a particular experience, they are asked to indicate how often they have this experience (sometimes, often, or nearly always), and how distressed they are by this experience (not distressed, a bit distressed, quite distressed, and very distressed). In the present research, weighted frequency scores for each symptom dimension were computed by summing the frequencies reported across all items that belong to the respective dimension; that is, "never"/"sometimes"/"often"/"nearly always" were recoded to $1 / 2 / 3 / 4$, and the total frequencies of positive, negative, and depressive symptoms were summed individually. Each of the total frequencies was then divided by the number of items answered for 
each respective dimension, to account for unanswered items and for the unequal number of questionnaire items across the three symptom dimensions. Therefore, each symptom dimension score is a weighted frequency score, such that the lowest possible score is 1.00 (responding "never" on all questions answered) and the highest possible score is 4.00 (responding "nearly always" on all questions answered). Examples of items in the CAPE are presented in Figure 2.1.

Please read the questions and circle the answers that best describe your experience.

1) Do you ever feel sad?

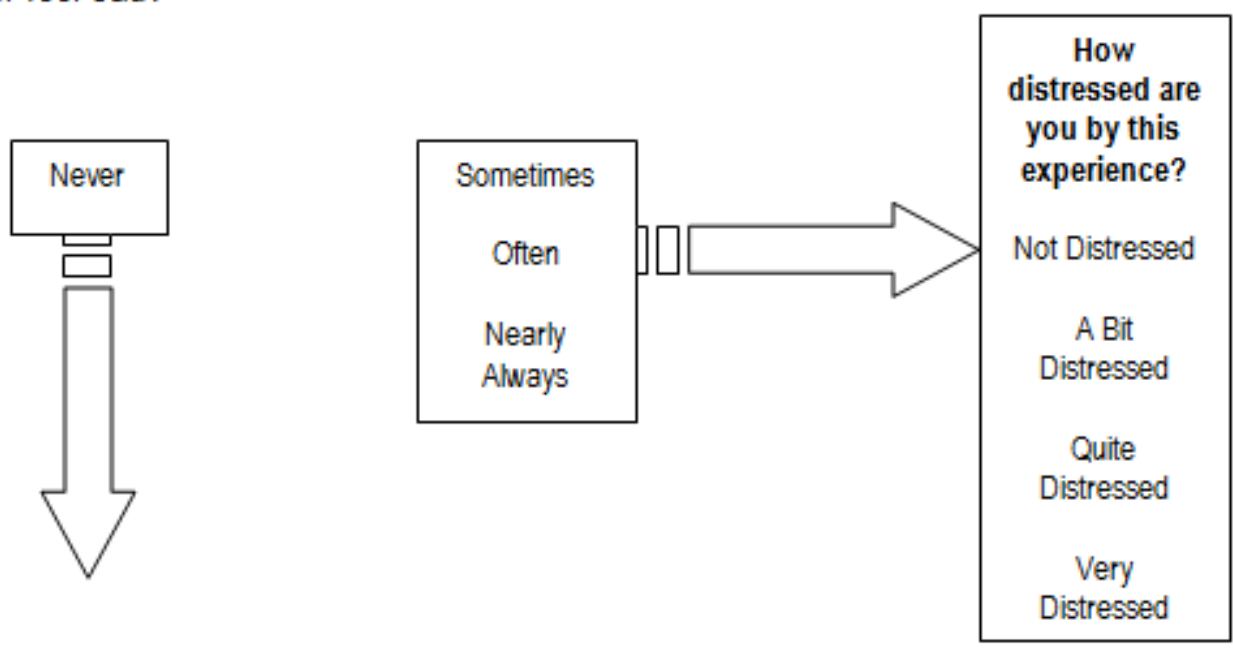

2) Do you ever feel as if people seem to drop hints about you or say things with a double meaning?

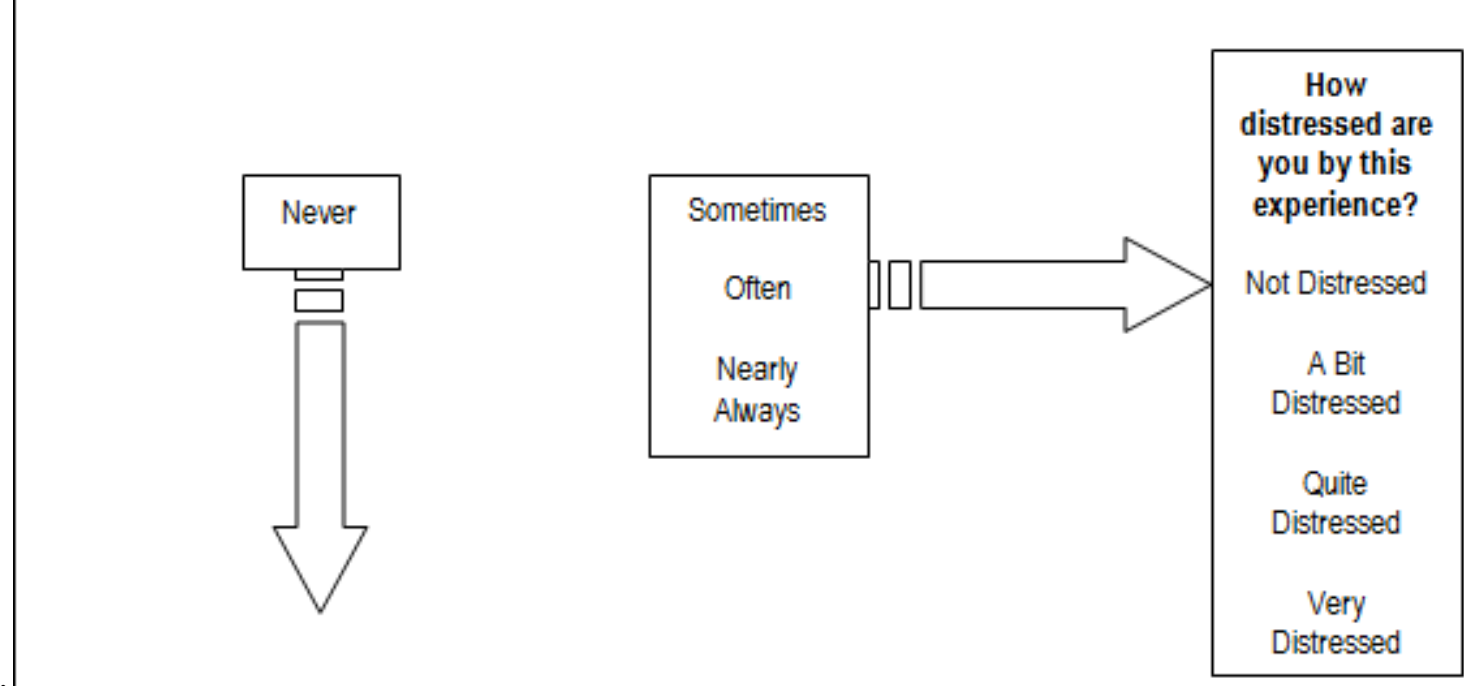

Figure 2.1. Two items from the CAPE questionnaire. 


\subsubsection{Attributional Style Bias Against Disconfirmatory Evidence (ASB) task}

As described in Section 1.5., the ASB paradigm combines that of the IPSAQ with the traditional BADE paradigm, incorporating a general assessment of attributional bias with an assessment of belief maintenance. The participant is first presented with a self-referent, positive or negative scenario, and is asked to rate the degree of responsibility of oneself, another person, and the situation. S/he may then revise these ratings after a second statement has been presented, which provides information about a possible cause.

The ASB task is computerized (screen shots of the task are provided in Figure 2.2. and Figure 2.3.), with a total of 20 scenarios presented (10 positive, 10 negative). The order of presentation for the scenarios was randomized for each participant, as well as the attribution implied in the second statement (e.g., "your team has won an important game" could be followed up by either "now imagine you played really well", "now imagine that your opponent played poorly", or "now imagine that the sun was in the other players' eyes the whole match"). The rating for each possible attribution was made on a continuous scale of 0 ("not responsible") to 10 ("very responsible"), in steps of 0.1 , with a small box to the right of each scale showing the current rating. The participant controlled the mouse and moved the scroll bars of each rating scale according to where s/he wanted them. Participants were provided with the following instructions at the start of the experiment:

In the following task, you will be asked to imagine yourself in a series of scenarios. In the top box of the screen you will be presented with two sentences, one at a time, describing a scenario. Each sentence will increase your knowledge about the scenario. After being presented with each sentence, your job is to determine the degree of responsibility You, an Other Person, and the Situation 
have for outcome of the presented scenario. You will be asked to rate the degree of responsibility you believe should be attributed to each source (i.e., You, an Other Person, and the Situation) after you read a given sentence in the top box. Each of the three sources must be considered independently from one another. Please do not compare the sources to each other. Instead, rate how well each one relates, on its own, to the information that is provided. As new information is provided, your task is to reconsider the degree of responsibility for a given outcome you have attributed to each source. You may change your ratings for each source as little or as much as you like, and one or more of the ratings can be the same if you feel that they are equally responsible for a given situation. You may want to keep some ratings the same, even after being given other event information. You will be controlling the mouse to make your ratings. After rating the sentences for Hint \#1, click on "Next" at the bottom of the screen to bring up the next hint. Do this after each set of ratings has been made. After establishing ratings to Hint \#2, click on "Finished".

The participant was then presented with practice trials to familiarize him or herself with the task. Once the participant had completed the practice trials, the experimenter provided the following instructions:

"Now that we have had some practice we will start the task. You will see three options, each with their own rating scale. You will be given two hints, one at a time, which will appear at the top of the screen. You will be asked to attribute how much responsibility you believe each of the three sources, You, an Other Person and the Situation has with respect to a given outcome. Once you are finished 
rating the degree of responsibility of all three sentences, press the next button to reveal the next hint."

Unlike the IPSAQ, participants were not asked to provide a causal explanation for the scenario before making their attributional ratings, as it has been demonstrated that independent raters' evaluations of participants' causal statements frequently do not match the participants' actual chosen attribution (Martin \& Penn, 2002; Randall, Corcoran, Day, \& Bentall, 2003). The test items were validated in a pilot study to ensure that the statements elicited reasonably plausible attribution ratings. Items in which participants showed a particularly unusual pattern of response (e.g., consistently high "other" attribution ratings where high "situation" attribution ratings would be expected) were removed from the task. Cronbach's alphas for the 10 positive scenarios in the present sample of participants $(n=171)$ were $\alpha=.862, \alpha=.815$, and $\alpha=.898$ for self, other-person, and situation attributions, respectively. Cronbach's alphas for the 10 negative scenarios were $\alpha=.799, \alpha=.836$, and $\alpha=.873$ for self, other-person, and situation attributions, respectively. 
You are offered a job.

Please rate the degree of responsibility for this outcome.
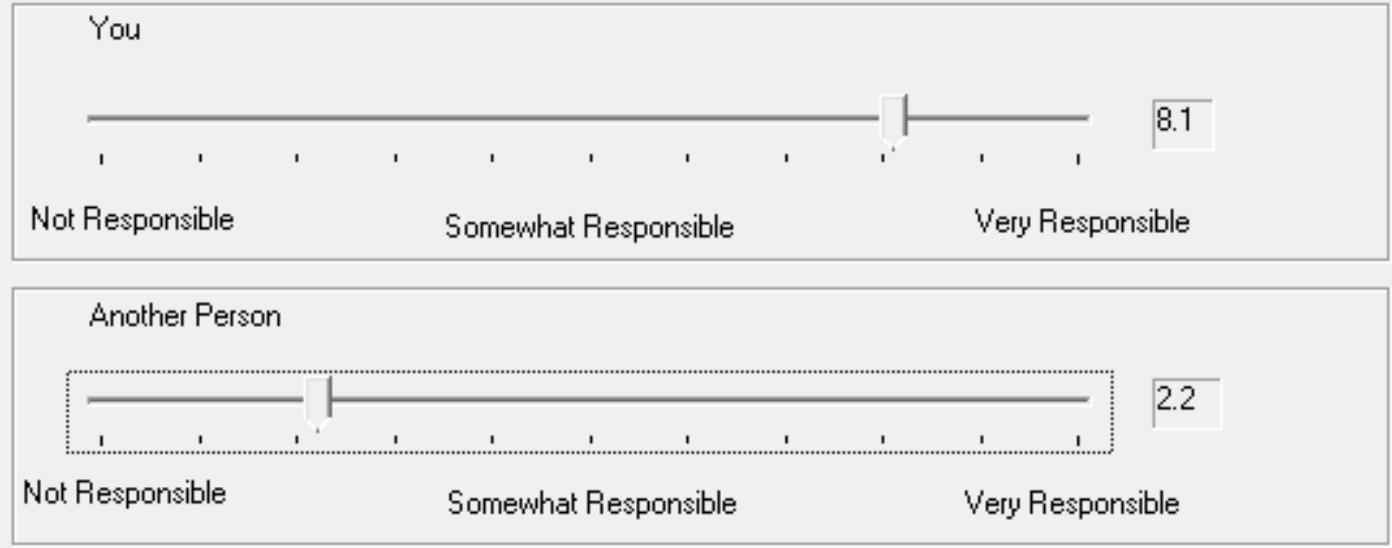

Situation.

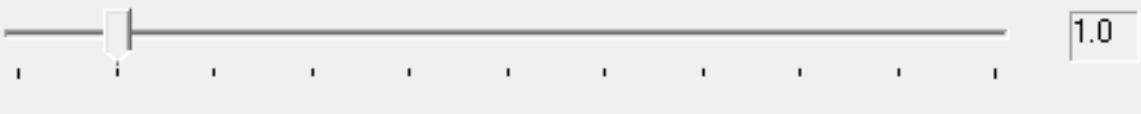

Not Responsible

Somewhat Responsible

Very Responsible

Figure 2.2. Screenshot of a trial from the ASB task after the first statement has been presented. 


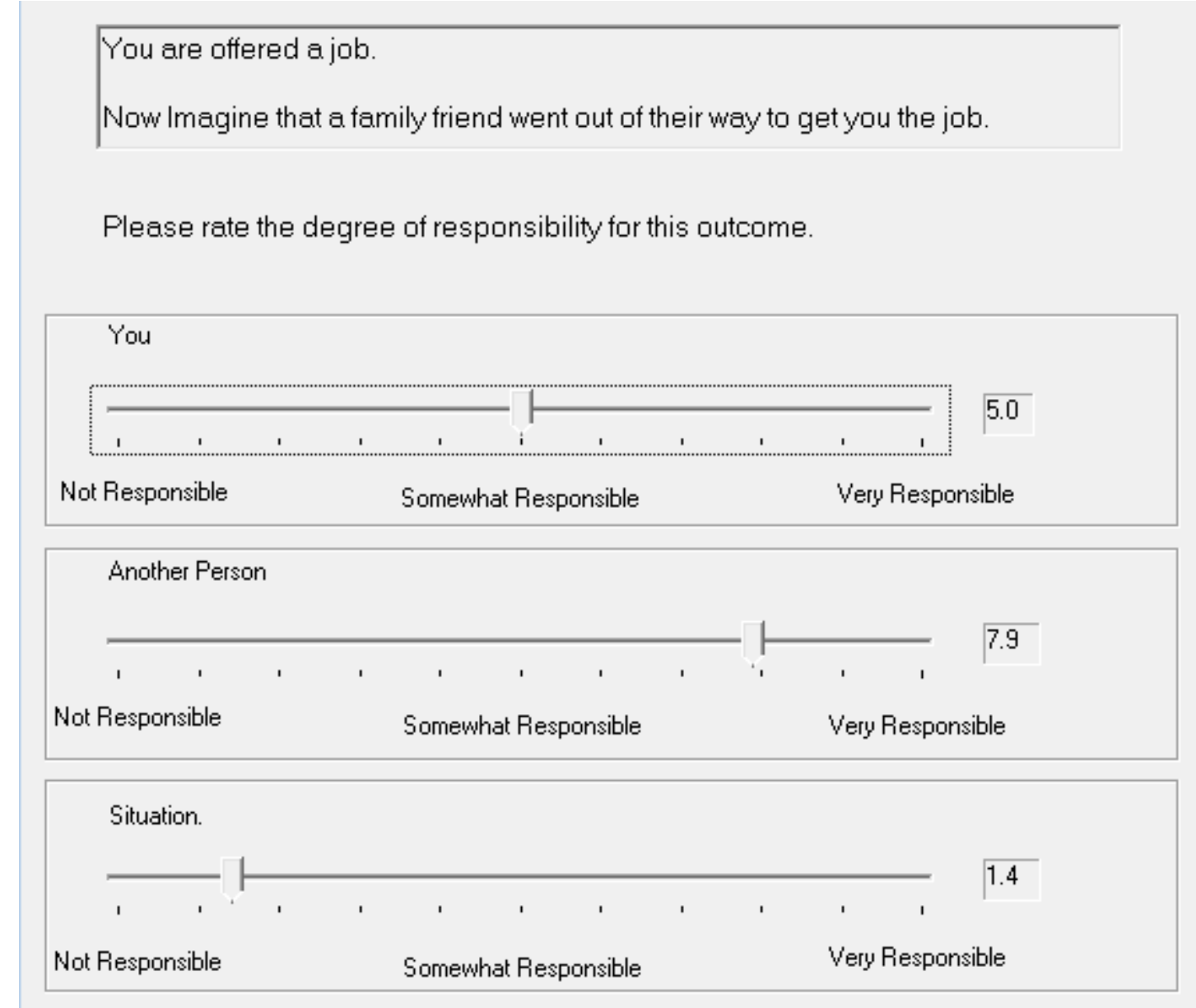

Figure 2.3. Screenshot of a trial from the ASB task after the second statement has been presented.

\subsection{Analysis}

The following statistical procedures were performed using MATLAB Version 7.6 (The MathWorks, 2008, Natick, MA; scripts presented in Appendix A) and GeSCA, a web-based software program developed for running generalized structured component analysis (http://www.sem-gesca.org). Mathematical operations are presented in Appendix B. IBM SPSS Statistics Version 19 (IBM Corporation, 2010, Armonk, NY) was used primarily for participant descriptives, t-tests, and analysis of variance (ANOVA) in Study 1. 


\subsubsection{Principal component analysis}

Principal component analysis (PCA) uses orthogonal transformation to convert a set of observed variables into a set of linearly uncorrelated variables called principal components, such that the first principal component accounts for the largest possible amount of variance in the data, and each succeeding component has the highest possible variance under the constraint that it is orthogonal to the preceding components. PCA is typically used for dimension reduction, as it allows a large set of variables to be reduced to a few principal components that reflect common variance among the variables that are most highly related to each component.

The number of components to retain is generally decided from the amount of variance each one accounts for, indicated by their respective eigenvalues (derived from either eigenvalue decomposition of the covariance or correlation matrix, or from squaring the singular values obtained from singular value decomposition of the mean centered and normalized data matrix). By convention, the components with eigenvalues $>1.0$ are all retained. Alternatively, the number of components to extract is determined by considering the plotted eigenvalues (i.e., the scree plot), which illustrates the extent to which each component contributes a meaningful and unique portion of variance (Cattell, 1966; Cattell \& Vogelmann, 1977). In the present research, the decision was generally based on the scree plot, as the eigenvalue $>1.0$ criterion can result in over-extraction (Bandalos \& Boehm-Kaufman, 2008; Cliff, 1988; Jackson, 1993), and does not apply when PCA is carried out on matrices of predicted scores, which have had a substantial amount of variance removed (as implicated in the present research; details provided in the following section). The extracted components are generally discussed in terms of component loadings (correlation coefficients indicating the extent to which each of the variables contributes to the component) and component scores (weighted composites of the standardized observed 
variables, with component weights derived from component loadings). If more than one component is extracted, the interpretability of the components can be increased by redistributing the variance (i.e., rotating the components). For example, varimax (orthogonal) rotation redistributes the variance captured by the original eigenvectors, stopping optimization when the matrix of component loadings optimally matches a so-called "simple structure", which is characterized by the following conditions: (1) any given variable has a high loading on a single component, and near-zero loadings on the others, and (2) any given component consists of only a few variables with very high loadings, with the remaining variables all having near-zero loadings.

In the present research, PCA was used to investigate cognitive processes engaged in the ASB task (reflected in the principal components), as well as to examine relationships between these cognitive processes and independent variables of interest (e.g., symptoms) by correlating component scores with these variables. Component scores are weighted aggregates that reflect variance common to a number of variables in a given dataset, and therefore are assumed to reflect individual differences in cognitive processes that cannot be adequately expressed by an individual test score. Previous studies using the traditional BADE task have similarly used PCA to identify and produce a measure of an individual's ability to integrate disambiguating evidence (i.e., "evidence integration", described in Section 1.4.), as reflected in a component that displays intercorrelations among disconfirmed interpretations (high positive loadings) and the confirmed interpretation (high negative loading; Sanford et al., 2014; Speechley et al., 2012).

\subsubsection{Constrained principal component analysis}

Along with identifying the higher-order cognitive operations engaged in the ASB task (reflected in its component structure), the present research aimed to determine whether any 
components may be directly predictable from group differences or symptom measures. This was accomplished using constrained principal component analysis (CPCA; Hunter \& Takane, 2002), an approach that combines multivariate multiple regression with PCA.

The first step in CPCA (i.e., the external analysis) involves partitioning the total variability in the criterion $(Z)$ into variance that can be explained by the predictor $(G)$ and variance that cannot be explained by the predictor, producing a matrix of predicted scores (the $G C$ matrix) and a matrix of residuals (the $E$ matrix). This is illustrated in the regression equation:

$$
Z=G C+E
$$

Where $Z=$ the dependent variable, $G=$ the independent variable, $C=$ the weights applied to $G$ to produce a matrix of predicted scores $(G C)$, and $E$ is a matrix of residuals.

The next step in CPCA (i.e., the internal analysis) is to apply PCA separately to each matrix (i.e., each source of variance) so that the resulting component solutions can be examined to determine which components of $Z$ are predictable and not predictable from $G$. This is achieved by correlating each component extracted from $G C$ with the independent variables in $G$, producing a matrix of $G$ "loadings" that indicate the degree to which each variable in $G$ is related to the corresponding components extracted from $G C$ (not to be confused with component loadings, as PCA is not necessarily run on $G$ ). The components extracted from $E$ reflect components of $Z$ that are not predictable from $G$; this component structure will closely resemble that of $Z$ if $G C$ explains only a small amount of the total variance in $Z$.

In the present research, attribution ratings from the ASB task were entered into $Z$, and an independent variable of interest (i.e., group membership, SSPI, or CAPE) was entered into $G$. In Study 1, which aimed to examine group differences on the ASB task, $G$ was comprised of indicator variables coding different groups of participants. In Study 2, $G$ was comprised of either 
SSPI or CAPE scores. As the ASB task assesses an individual's attributional biases when presented with ambiguous information as well as their willingness to adjust their attribution ratings after receiving (dis)confirming evidence, in most cases CPCA was first run with only the initial attribution ratings entered in $Z$ (as this part of the task is comparable to the IPSAQ) and then, to examine the cognitive operations that included the processing of (and responding to) confirming and disconfirming evidence, CPCA was performed again with initial and final ratings entered into one $Z$ matrix. In solutions where more than one component was extracted, the components were rotated using varimax rotation with Kaiser normalization.

\subsubsection{Generalized structured component analysis}

Generalized structured component analysis (GSCA; Hwang \& Takane, 2004), a component-based method of structural equation modelling (SEM), was employed in Study 1 (Chapter 3) to verify that the component structure of the set of ASB items was comparable between healthy individuals, schizophrenia patients, and bipolar disorder patients. Similar to PCA, GSCA computes latent variables as weighted composites of standardized observed variables, which are optimized to explain the maximum proportion of variance according to the specified model. One advantage of the use of components rather than factors in SEM is that this prevents improper solutions such as negative variance estimates. Further, GSCA does not rely on strict distributional assumptions such as multivariate normality of observed data (which is likely to be violated on many of the variables in the ASB task), and can employ complex models even with small sample sizes. As well as providing a measure of model fit (FIT) in a single sample of participants, GSCA is able to perform multi-group analyses by employing a global optimization procedure that allows for between-group comparisons of a proposed model. 
GSCA provides a global value of model fit (FIT), which ranges from 0 to 1 (though typically no more than .6 and no less than .3 ) and indicates how much of the total variance in the observed data is explained by the specified model. Like PCA, component loadings for each observed variable (i.e., indicator) specify how well the indicator loads onto its respective component. Also like PCA, component scores (or latent variable scores) are computed by applying weights to their respective indicators to produce unique latent variable scores for each participant in a given dataset. Significance values for these parameters are obtained by dividing a parameter estimate (e.g., a given component loading) by its bootstrap standard error (SE), which produces a bootstrap critical ratio (CR) value (Efron, 1981). A parameter estimate may be considered significant at a .05 level if its bootstrap CR is equal to or greater than 2 in absolute value, under the assumption that the bootstrap distribution of the estimate is roughly normal.

As noted, GSCA is able to employ multi-group analyses to examine how well a specified model fits across different groups of participants. Component loadings can be constrained to be identical across multiple groups of participants, or they can be unconstrained (i.e., free to vary between groups). The constrained model will usually have a lower FIT than the unconstrained model because loadings from a solution that is not specifically optimized for each group of participants are imposed on the data for all groups; however, if this difference is not significant, then it can be assumed that the model is comparable between groups. This is accomplished using a bootstrapped $t$-test; that is, the constrained and unconstrained models are fit to each of $n$ bootstrap samples, which are drawn randomly from the original dataset with replacement. The differences between FIT values are calculated, then a one-sample $t$-test is run on the difference scores using degrees of freedom $=n-1$ to determine whether the mean difference score is significantly different from zero. If it is not statistically significant from zero, then it can be 
assumed that there is no reliable mean difference in FIT between the constrained and unconstrained models, and by extension, there are no significant differences between groups with respect to the model that commonly fits all groups.

In the present research, GSCA was used to test a proposed structure for the ASB task based on an exploratory PCA, as described in Section 2.3.1. Although GSCA can be used for more advanced model testing (e.g., path analysis), for the purposes of the present research, it was used simply to test whether a proposed component structure of the ASB task was comparable across groups (i.e., schizophrenia patients, bipolar disorder patients, and healthy individuals). PCA is highly sensitive to particular idiosyncrasies of the sample under investigation, so although a large sample of participants is ideal to reduce error, it is important to check that the component structure does not change in important ways in smaller samples with unique characteristics (such as a sample of patients with mental illness). Multi-group GSCA helps verify that the observed task structure is generalizable and arises from the task demands instead of from differences between groups included in the pooled sample of participants. This method is employed in Study 1, as reported in the following chapter. 


\section{Chapter 3: Attributional Biases on the ASB Task (Study 1)}

\subsection{Aims}

As described in the previous chapter, PCA can help identify higher-order cognitive processes engaged in a behavioural task by constructing components from patterns of intercorrelations among the task variables. This allows for individual differences in these cognitive processes to be quantified in component scores, which can then be used to examine performance on the task. The following study addresses the first two objectives described in Chapter 1; that is, to (1) determine the cognitive processes engaged in the ASB task by establishing its component structure, and (2) determine the extent to which differences between healthy individuals, schizophrenia patients, bipolar disorder patients, and symptom-based patient subgroups (based on severity of paranoid delusions or depression) account for performance on the ASB task.

\subsection{Predictions}

General predictions were described in Section 1.6. In terms of specific components expected to arise, an "evidence integration" component - which in the BADE task reflects the degree to which individuals are willing to down-rate the plausibility of disconfirmed interpretations and up-rate the "true" interpretation - may arise in the ASB task as displayed by high positive loadings for disconfirmed attributions and high negative loadings for confirmed attributions. Further, such a component could be particular to certain attributional loci, reflecting specific attributional biases that are not amenable to change following disconfirming information.

The first segment of the ASB task is analogous to the IPSAQ (Section 1.3.1.); that is, a single statement describes a scenario in which the cause is unknown, and the participants' 
attribution ratings are based on their own formulations of the scenario rather than on explicit information provided. In general terms, it was predicted that a self-serving bias would be observed for the full sample of participants. In line with the defense model of paranoia, it was also predicted that the self-serving bias and/or a personalizing bias (blaming other people as opposed to the situation), would be enhanced in the paranoid group compared with non-paranoid, bipolar, and healthy participants. By contrast, the self-serving bias was expected to be attenuated in severely depressed patients compared to non-depressed patients and healthy individuals.

In addition to displaying a self-serving and/or personalizing bias in their initial attribution ratings, the paranoid group was expected to show a relative bias against evidence disconfirming self-serving and/or personalizing attributions compared to the other three groups. In terms of components specifically emerging from group differences in attribution ratings, this could be reflected in a component dominated by high negative loadings for confirmed self attributions and high positive loadings for disconfirmed other-person attributions for negative scenarios, or some similar pattern reflecting (un)willingness to revise self-serving and/or personalizing attributions. Depressed patients were not expected to display a BADE, as this bias is thought to be specifically associated with delusions.

\subsection{Methods}

\subsubsection{Participants}

58 healthy controls, 44 schizophrenia patients, and 48 bipolar disorder patients were included in the following analyses, with inclusion/exclusion criteria applied as described in Section 2.1. Table 3.1. presents a list of sample characteristics for the three groups, including gender distribution, IQ (using the KBIT-II composite score), years of education, years of age, and socioeconomic status (SES, determined using a standard demographic questionnaire). 
Paranoid and non-paranoid patient subgroups were formed by dividing the schizophrenia patients based on severity of paranoid delusions (paranoid group $\geq 3$ on SSPI paranoid delusions measure; $n=10$ paranoid, $n=31$ non-paranoid). In analyses that included depressed and nondepressed patient subgroups, schizophrenia patients and bipolar disorder patients were pooled and then divided based on severity of depression (depressed group $\geq 3$ on SSPI depression measure; $n=14$ depressed, $n=74$ non-depressed). Bipolar disorder patients were not pooled with schizophrenia patients for the paranoid/non-paranoid groupings because delusions are more specific to schizophrenia, and indeed, only two bipolar disorder patients had paranoid delusions scores $\geq 3$. However, the number of severely depressed patients in each of the diagnostic groups was approximately the same ( $n=6 / 41$ schizophrenia patients, $n=8 / 47$ bipolar disorder patients).

Table 3.1. Study 1 sample means and standard deviations (unless otherwise specified) for demographic variables and IQ.

\begin{tabular}{lcccc}
\hline Variables & Healthy $^{\mathrm{a}}$ & Schizophrenia $^{\mathrm{b}}$ & Bipolar $^{\mathrm{c}}$ & All $^{\mathrm{d}}$ \\
\hline Gender $(\mathrm{m} / \mathrm{f})$ & $30 / 28$ & $27 / 17$ & $19 / 29$ & $76 / 74$ \\
KBIT IQ & $106.16(9.52)$ & $102(13.59)$ & $100.34(10.57)$ & $103.1(11.37)$ \\
Years of education & $15.92(1.84)$ & $13.6(2.74)$ & $14.23(1.97)$ & $14.7(2.39)$ \\
Years of Age & $29.81(8.85)$ & $36.32(9.4)$ & $35.85(11.05)$ & $33.65(10.17)$ \\
SES & $61.43(17.14)$ & $75.6(27.47)$ & $65.06(19.8)$ & $66.63(22)$ \\
\hline
\end{tabular}

${ }^{a} n=58 .{ }^{\mathrm{b}} n=44 .{ }^{\mathrm{c}} n=48 .{ }^{\mathrm{d}} n=150$.

\subsubsection{Procedures}

\section{Aim no. 1 (task structure)}

An exploratory PCA was performed on all initial and final attribution ratings to examine components of the ASB task that include the processing of (dis)confirming evidence. "Nonmatching" ratings (for example, final self attribution ratings for scenarios in which an otherperson or situational cause had been implied in the "evidence" provided) were averaged for each attribution, for positive and for negative scenarios. This produced a total of 18 variables of 
interest in the ASB task: 6 initial ratings (self/other/situation ratings for positive/negative scenarios) plus 12 final ratings [3 attributions (self/other/situation) $\times 2$ types of scenarios (positive/negative) $\times 2$ levels of agreement (match/non-match with the attribution implied)] Initial attribution ratings were labelled "unconfirmed" attributions, ratings that matched the attribution implied were labelled "confirmed" attributions, and the averaged non-matching ratings were labelled "disconfirmed" attributions. Multi-group generalized structured component analysis (GSCA, described in Section 2.3.3.) was employed to verify that the proposed latent variable structure (based on results from the exploratory PCA) explained the data well and was appropriate for each of the three diagnostic groups (healthy, schizophrenia, bipolar disorder).

\section{Aim no. 2 (task performance)}

The planned approach to Aim no. 2 was to run three experiments with constrained PCA (CPCA, described in Section 2.3.2.), with various participant groupings employed in line with previous studies of attributional style in paranoia and depression, to determine the percentage of variance explained by group differences as well as the component patterns that emerge from this variance. In all analyses, the ASB task was entered as the criterion/dependent variable $(Z)$ and a matrix of indicator variables was entered as the predictor/independent variable $(G)$. The first analysis used broad diagnostic-based groupings as a predictor (i.e., healthy individuals, schizophrenia patients, and bipolar disorder patients). The second analysis employed healthy, bipolar disorder, paranoid schizophrenia, and non-paranoid schizophrenia participant groupings. Lastly, the third analysis employed healthy, depressed, and non-depressed participant groupings. However, group differences accounted for a negligible amount of variance as revealed by the external analyses (i.e., multivariate multiple regressions) for all comparisons, and so the internal analyses (i.e., PCAs) were not performed on the resulting $G C$ or $E$ matrices. Examination of 
within-subject effects for initial attribution ratings was completed with repeated measures analysis of variance (ANOVA) in the full sample of participants.

\subsection{Results}

\subsubsection{Participant characteristics}

The groups did not differ in gender distribution, $\chi^{2}(2)=4.40, p>.10$. However, there were group differences in IQ $\left(F(2,145)=3.82, p=.024, \eta^{2}=.05\right)$, years of education $(F(2,147)$ $\left.=15.82, p<.001, \eta^{2}=.18\right)$, age $\left(F(2,147)=7.36, p=.001, \eta^{2}=.09\right)$, and $\operatorname{SES}(F(2,145)=$ $\left.5.55, p=.005, \eta^{2}=.07\right)$. The schizophrenia group tended to have higher SSPI symptom scores than the bipolar disorder group, especially for reality distortion symptoms (delusions and hallucinations), as listed in Table 3.2.

Post hoc comparisons using the Tukey HSD test revealed a significant difference in IQ between the healthy control group $(M=106.16, S D=9.52,95 \% C I[103.65,108.66])$ and the bipolar patients $(M=100.34, S D=10.57,95 \% C I[97.24,103.44])$, but no differences between either group and the schizophrenia patients $(M=102.00, S D=13.59,95 \% C I[97.82,106.18])$. For years of education, post hoc comparisons revealed a significant difference between the healthy controls $(M=15.92, S D=1.84,95 \% C I[15.44,16.41])$ and both the bipolar group $(M=$ $14.23, S D=1.97,95 \% C I[13.66,14.80])$ and schizophrenia group $(M=13.60, S D=2.74,95 \%$ $C I[12.77,14.43])$. For years of age, post hoc comparisons revealed that the healthy controls were significantly younger $(M=29.81, S D=8.85,95 \% C I[27.48,32.14])$ than both the bipolar group $(M=35.85, S D=11.05,95 \% C I[32.65,39.06])$ and schizophrenia group $(M=36.32, S D$ $=9.40,95 \%$ CI $[33.46,39.18])$. Finally, for SES, post hoc comparisons revealed a significant difference between the healthy controls $(M=61.43, S D=17.14,95 \% C I[56.93,65.94])$ and schizophrenia patients $(M=75.60, S D=27.47,95 \% C I[67.03,84.16])$. Neither group differed 
from bipolar patients $(M=65.06, S D=19.80,95 \% C I[59.31,70.81])$. Although there was a trend difference between schizophrenia and bipolar patients for SES $(p=.054)$, these groups did not differ on any other measures (all $p \mathrm{~s}>.30$ ).

Although gender distribution did not differ between groups, possible effects of gender on attributional style were investigated to determine whether gender should be taken into account for analyses of the ASB task. A 2 (positive/negative scenario) $\times 3$ (self/other/situation rating) $\times 2$ (male/female) analysis of variance (ANOVA) was performed with initial ASB ratings. There were no within-subject interactions for scenario $\times$ gender, $F(1,148)=0.41, p>.50$, attribution $\times$ gender, $F(1.78,262.98)=0.19, p>.80$, or scenario $\times$ attribution $\times$ gender, $F(1.67,247.31)=$ $1.14, p>.30$. Finally, there was no main effect of gender, $F(1,148)=0.03, p>.80$. Therefore, gender will not be further investigated. 
Table 3.2. Study 1 mean SSPI symptom scores for schizophrenia patients and bipolar disorder patients (standard deviations in parentheses).

\begin{tabular}{lccc}
\hline Symptom & Schizophrenia $^{\mathrm{a}}$ & Bipolar disorder $^{\mathrm{b}}$ & All $^{\mathrm{c}}$ \\
\hline Anxiety & $1.30(1.00)$ & $1.33(1.14)$ & $1.32(1.07)$ \\
Depression & $1.02(1.25)$ & $1.10(1.24)$ & $1.07(1.24)$ \\
Anhedonia & $1.05(1.18)$ & $0.98(1.10)$ & $1.01(1.13)$ \\
Elated mood & $0.39(0.78)$ & $0.69(1.06)$ & $0.54(0.94)$ \\
Insomnia & $0.95(1.24)$ & $1.23(1.19)$ & $1.10(1.21)$ \\
Somatic complaints & $0.20(0.59)$ & $0.10(0.31)$ & $0.15(0.47)$ \\
Delusions - general & $1.98(1.36)$ & $0.79(1.17)$ & $1.36(1.39)^{* * *}$ \\
Delusions - guilt/worthlessness & $0.43(0.87)$ & $0.15(0.55)$ & $0.28(0.73)$ \\
Delusions - grandiose & $0.89(1.24)$ & $0.33(0.88)$ & $0.60(1.10)^{*}$ \\
Delusions - paranoid & $1.34(1.46)$ & $0.50(0.97)$ & $0.90(1.29)^{* *}$ \\
Delusions - Schneiderian & $0.86(1.27)$ & $0.23(0.72)$ & $0.53(1.06)^{* *}$ \\
Hallucinations - general & $1.43(1.80)$ & $0.29(0.92)$ & $0.84(1.51)^{* * *}$ \\
Hallucinations $-2^{\text {nd }}$ person & $0.93(1.66)$ & $0.23(0.83)$ & $0.57(1.34)^{*}$ \\
Hallucinations - Schneiderian & $0.84(1.58)$ & $0.21(0.82)$ & $0.51(1.28)^{*}$ \\
Impaired attention & $1.30(1.00)$ & $1.31(0.88)$ & $1.30(0.93)$ \\
Disorientation & $0.07(0.25)$ & $0.00(0.00)$ & $0.03(0.18)$ \\
Overactivity & $0.82(1.04)$ & $1.02(0.98)$ & $0.92(1.01)$ \\
Underactivity & $1.14(1.15)$ & $0.85(0.92)$ & $0.99(1.04)$ \\
Flattened affect & $1.20(1.02)$ & $0.63(0.89)$ & $0.90(1.00)^{* *}$ \\
Inappropriate affect & $0.11(0.49)$ & $0.06(0.32)$ & $0.09(0.41)$ \\
Pressured speech & $0.30(0.70)$ & $0.31(0.62)$ & $0.30(0.66)$ \\
Poverty of speech & $0.39(0.72)$ & $0.13(0.44)$ & $0.25(0.60)^{*}$ \\
Disordered thought & $0.32(0.80)$ & $0.06(0.32)$ & $0.18(0.61)$ \\
Peculiar behaviour & $0.20(0.59)$ & $0.04(0.20)$ & $0.12(0.44)$ \\
Irritability/hostility & $0.39(0.69)$ & $0.29(0.50)$ & $0.34(0.60)$ \\
Impaired insight & $1.25(1.16)$ & $0.77(1.02)$ & $1.00(1.11)^{*}$ \\
Total (excluding subscales) & $15.80(8.12)$ & $12.00(5.92)$ & $13.82(7.27)^{*}$ \\
\hline a & & &
\end{tabular}

${ }^{\mathrm{a}} n=44 .{ }^{\mathrm{b}} n=48 .{ }^{\mathrm{c}} n=92$.

${ }^{*} p<.05, * * p<.01,{ }^{* * *} p<.001$ for independent samples t-tests between the two patient groups.

\subsubsection{Task structure}

The component structure of the ASB task was investigated in the full sample of participants $(n=150)$. Mean attribution ratings for each group and for the full sample are listed in Table 3.3. below. 
Table 3.3. Mean attribution ratings for healthy, schizophrenia, and bipolar disorder participant groups. Standard deviations in parentheses.

\begin{tabular}{lcccc}
\hline Attributions & Healthy $^{\mathrm{a}}$ & Schizophrenia $^{\mathrm{b}}$ & Bipolar $^{\mathrm{c}}$ & Total $^{\mathrm{d}}$ \\
\hline Self unconfirmed, pos & $6.86(1.61)$ & $6.78(2.30)$ & $6.53(2.00)$ & $6.73(1.95)$ \\
Oth unconfirmed, pos & $4.21(1.41)$ & $3.91(2.54)$ & $3.68(1.48)$ & $3.95(1.84)$ \\
Sit unconfirmed, pos & $4.37(2.26)$ & $4.96(2.70)$ & $4.21(2.11)$ & $4.49(2.36)$ \\
Self unconfirmed, neg & $3.68(1.47)$ & $3.92(2.20)$ & $3.28(1.75)$ & $3.62(1.80)$ \\
Oth unconfirmed, neg & $6.36(1.71)$ & $5.67(2.32)$ & $5.89(2.02)$ & $6.01(2.01)$ \\
Sit unconfirmed, neg & $4.45(2.14)$ & $4.76(2.50)$ & $4.05(1.97)$ & $4.41(2.20)$ \\
\hline Self confirmed, pos & $8.70(1.24)$ & $8.72(1.93)$ & $8.56(1.87)$ & $8.66(1.66)$ \\
Self disconfirmed, pos & $5.20(1.74)$ & $5.50(2.50)$ & $5.26(2.01)$ & $5.30(2.06)$ \\
Self confirmed, neg & $8.33(1.86)$ & $8.20(2.36)$ & $7.44(2.67)$ & $8.00(2.31)$ \\
Self disconfirmed, neg & $2.64(1.72)$ & $2.88(2.32)$ & $2.41(1.84)$ & $2.63(1.94)$ \\
\hline Oth confirmed, pos & $8.14(1.6)$ & $7.77(2.32)$ & $7.89(2.18)$ & $7.95(2.02)$ \\
Oth disconfirmed, pos & $4.82(1.72)$ & $4.25(2.73)$ & $4.44(1.70)$ & $4.53(2.06)$ \\
Oth confirmed, neg & $8.35(1.77)$ & $7.88(2.83)$ & $8.37(2.19)$ & $8.22(2.25)$ \\
Oth disconfirmed, neg & $4.29(1.69)$ & $3.63(2.16)$ & $4.20(2.07)$ & $4.07(1.97)$ \\
\hline Sit confirmed, pos & $7.47(2.34)$ & $7.69(2.29)$ & $7.12(2.32)$ & $7.42(2.32)$ \\
Sit disconfirmed, pos & $4.92(2.30)$ & $5.67(2.78)$ & $4.82(2.46)$ & $5.11(2.51)$ \\
Sit confirmed, neg & $7.80(2.21)$ & $7.38(2.26)$ & $7.01(2.81)$ & $7.42(2.44)$ \\
Sit disconfirmed, neg & $4.25(2.31)$ & $5.01(2.79)$ & $3.96(2.43)$ & $4.38(2.51)$ \\
\hline
\end{tabular}

Note: $\mathbf{p o s}=$ positive scenario; neg $=$ negative scenario; oth $=$ other-person attribution; sit $=$ situation attribution. Unconfirmed attributions are the initial ratings provided following the first statement presented; confirmed attributions are the final ratings provided when the respective attributional locus was supported by the preceding evidence; disconfirmed attributions are the final ratings provided when the respective attributional locus was not supported by the evidence.

${ }^{\mathrm{a}} n=58 .{ }^{\mathrm{b}} n=44 .{ }^{\mathrm{c}} n=48 .{ }^{\mathrm{d}} n=150$.

\section{Principal Component Analysis (PCA)}

PCA revealed a four-component solution for the ASB task, accounting for $73.43 \%$ of the total variance in attribution ratings. These components were dominated by: (1) unconfirmed and disconfirmed situation attributions (22.20\% of the total variance), (2) confirmed attributions (22.17\% of total), (3) unconfirmed and disconfirmed other-person attributions (15.83\% of total), and (4) unconfirmed and disconfirmed self attributions (13.23\% of total). The loadings for each component are listed in Table 3.4. 
Note that unconfirmed and disconfirmed attributions for each attributional locus loaded together on one component, distinct from confirmed attributions, and with each attributional locus dominating one component. Self, other, and situation attributions that are unconfirmed and disconfirmed can all be considered unsupported by the available evidence, and so are hereafter labelled "unsupported". Therefore, it appears that the 18 ASB task variables may be reduced to four components, dominated by ratings for: (1) unsupported situation attributions, (2) supported (i.e., confirmed) attributions, (3) unsupported other-person attributions, and (4) unsupported self attributions.

Table 3.4. Component loadings for the ASB task, with four components extracted from PCA.

\begin{tabular}{lcccc}
\hline Attribution & $\begin{array}{c}\text { Unsupported } \\
\text { Situation }\end{array}$ & $\begin{array}{c}\text { Supported } \\
\text { (All) }\end{array}$ & $\begin{array}{c}\text { Unsupported } \\
\text { Other }\end{array}$ & $\begin{array}{c}\text { Unsupported } \\
\text { Self }\end{array}$ \\
\hline Sit unconfirmed, pos & $\mathbf{0 . 8 4 6}$ & 0.067 & 0.177 & 0.202 \\
Sit unconfirmed, neg & $\mathbf{0 . 8 2 7}$ & 0.219 & 0.242 & 0.200 \\
Sit disconfirmed, pos & $\mathbf{0 . 8 6 8}$ & 0.113 & 0.110 & 0.145 \\
Sit disconfirmed, neg & $\mathbf{0 . 8 4 6}$ & -0.028 & 0.228 & 0.117 \\
Self confirmed, pos & 0.013 & $\mathbf{0 . 6 9 6}$ & 0.149 & 0.510 \\
Self confirmed, neg & 0.008 & $\mathbf{0 . 7 6 0}$ & -0.095 & 0.118 \\
Oth confirmed, pos & -0.026 & $\mathbf{0 . 7 7 3}$ & 0.387 & 0.042 \\
Oth confirmed, neg & -0.107 & $\mathbf{0 . 8 2 6}$ & 0.344 & 0.036 \\
Sit confirmed, pos & 0.491 & $\mathbf{0 . 6 7 8}$ & 0.011 & -0.100 \\
Sit confirmed, neg & 0.358 & $\mathbf{0 . 7 3 0}$ & -0.010 & -0.125 \\
Oth unconfirmed, pos & 0.283 & 0.193 & $\mathbf{0 . 7 4 3}$ & 0.160 \\
Oth unconfirmed, neg & 0.011 & 0.496 & $\mathbf{0 . 7 1 7}$ & 0.212 \\
Oth disconfirmed, pos & 0.305 & 0.112 & $\mathbf{0 . 7 8 2}$ & 0.097 \\
Oth disconfirmed, neg & 0.183 & -0.082 & $\mathbf{0 . 7 4 1}$ & 0.250 \\
Self unconfirmed, pos & 0.110 & 0.443 & 0.279 & $\mathbf{0 . 6 8 5}$ \\
Self unconfirmed, neg & 0.472 & 0.088 & 0.135 & $\mathbf{0 . 6 4 9}$ \\
Self disconfirmed, pos & 0.176 & -0.032 & 0.214 & $\mathbf{0 . 8 0 9}$ \\
Self disconfirmed, neg & 0.523 & -0.290 & 0.163 & $\mathbf{0 . 5 2 5}$ \\
\hline
\end{tabular}

Note : $\mathbf{p o s}=$ positive scenario; $\mathbf{n e g}=$ negative scenario; sit $=$ situation; oth $=$ other. Unconfirmed attributions are the initial ratings provided following the first statement presented; confirmed attributions are the final ratings provided when the respective attributional locus was supported by the preceding evidence; disconfirmed attributions are the final ratings provided when the respective attributional locus was not supported by the evidence. The "Unsupported" Situation, Other, and Self components were specifically dominated by unsupported (i.e., unconfirmed and disconfirmed) attributions for each respective attributional locus, while the "Supported" component consisted of all confirmed attributions for all attributional loci. 
In an analysis of the traditional BADE task, a "conservatism" component emerged that was apparently driven by individuals rating low for interpretations in which high ratings would be justified (i.e., initial "lure" interpretations and the "true" interpretation; Speechley et al., 2012), which was revealed through an examination of scatter plots of the ratings that had high loadings on this component. Given the high contributions of confirmed attributions driving the "supported" component revealed in the present analysis, a similar investigation of scatter plots of these ratings was performed. However, none of the intercorrelations appeared to be driven by low raters, and so it cannot be concluded that this "supported" component is analogous to the "conservatism" component observed in the BADE task.

\section{Generalized Structured Component Analysis (GSCA)}

The proposed model consisted of four latent variables, reflecting the four components described above such that the indicators entered into their respective variables consisted of (1) supported attributions, (2) unsupported self attributions, (3) unsupported other-person attributions, and (4) unsupported situation attributions. No specifications regarding relationships between the four latent variables were entered into the model.

The overall FIT of the unconstrained model $=0.545$, suggesting that the model explains the observed data well. The component loadings generated for each group are listed in Table 3.5; almost all of these were significant, with the exception of unconfirmed self attributions for positive scenarios on the "unsupported self" latent variable in the healthy control group.

When cross-group equality constraints were imposed on the component loadings, the overall FIT of the model $=0.543$. Table 3.6. lists the loadings generated; all of these were statistically significant. Although the unconstrained model had a slightly higher FIT (0.545), this 
difference was non-significant, $t(99)=1.22, p=.225$. Therefore, it can be assumed that the model is appropriate for all three groups of participants. 
Table 3.5. Component loadings for each latent variable and each group from unconstrained multi-group GSCA.

\begin{tabular}{|c|c|c|c|c|c|c|c|}
\hline \multirow[b]{2}{*}{ Latent Variable } & \multirow[b]{2}{*}{ Indicator } & \multicolumn{2}{|c|}{ Healthy $(n=58)$} & \multicolumn{2}{|c|}{ Schizophrenia $(n=44)$} & \multicolumn{2}{|c|}{ Bipolar $(n=48)$} \\
\hline & & Loading & $\mathrm{CR}$ & Loading & $\mathrm{CR}$ & Loading & $\mathrm{CR}$ \\
\hline \multirow{6}{*}{$\begin{array}{l}\text { Supported } \\
(\mathrm{AVE}=0.614)\end{array}$} & Self, pos & 0.841 & $17.63^{*}$ & 0.702 & $4.57^{*}$ & 0.750 & $6.18^{*}$ \\
\hline & Self, neg & 0.699 & $8.24^{*}$ & 0.838 & $13.30^{*}$ & 0.707 & $5.17^{*}$ \\
\hline & Other, pos & 0.764 & $10.29^{*}$ & 0.891 & $24.53^{*}$ & 0.837 & $17.46^{*}$ \\
\hline & Other, neg & 0.835 & $15.09^{*}$ & 0.887 & $27.28^{*}$ & 0.911 & $23.56^{*}$ \\
\hline & Situation, pos & 0.799 & $16.38^{*}$ & 0.584 & $2.87^{*}$ & 0.775 & $6.72^{*}$ \\
\hline & Situation, neg & 0.780 & $10.25^{*}$ & 0.786 & $11.77^{*}$ & 0.670 & $4.47^{*}$ \\
\hline \multirow{4}{*}{$\begin{array}{l}\text { Self }- \text { Unsupported } \\
(\mathrm{AVE}=0.596)\end{array}$} & Unconfirmed, pos & 0.405 & 1.17 & 0.798 & $12.82^{*}$ & 0.733 & $8.20^{*}$ \\
\hline & Unconfirmed, neg & 0.826 & $19.56^{*}$ & 0.852 & $15.27^{*}$ & 0.887 & $27.59^{*}$ \\
\hline & Disconfirmed, pos & 0.758 & $4.09^{*}$ & 0.827 & $15.51^{*}$ & 0.840 & $19.65^{*}$ \\
\hline & Disconfirmed, neg & 0.739 & $7.15^{*}$ & 0.794 & $8.66^{*}$ & 0.736 & $6.11^{*}$ \\
\hline \multirow{4}{*}{$\begin{array}{l}\text { Oth - Unsupported } \\
(\mathrm{AVE}=0.655)\end{array}$} & Unconfirmed, pos & 0.860 & $20.24^{*}$ & 0.904 & $43.69^{*}$ & 0.770 & $10.08^{*}$ \\
\hline & Unconfirmed, neg & 0.801 & $10.76^{*}$ & 0.858 & $22.34^{*}$ & 0.800 & $12.05^{*}$ \\
\hline & Disconfirmed, pos & 0.849 & $22.89^{*}$ & 0.894 & $33.50^{*}$ & 0.723 & $6.56^{*}$ \\
\hline & Disconfirmed, neg & 0.724 & $9.39^{*}$ & 0.779 & $7.80^{*}$ & 0.737 & $10.17^{*}$ \\
\hline \multirow{4}{*}{$\begin{array}{l}\text { Sit - Unsupported } \\
(\mathrm{AVE}=0.816)\end{array}$} & Unconfirmed, pos & 0.938 & $47.86^{*}$ & 0.920 & $38.49^{*}$ & 0.904 & $32.93^{*}$ \\
\hline & Unconfirmed, neg & 0.915 & $28.84^{*}$ & 0.886 & $23.47^{*}$ & 0.902 & $37.18^{*}$ \\
\hline & Disconfirmed, pos & 0.928 & $44.12^{*}$ & 0.864 & $20.50^{*}$ & 0.927 & $58.15^{*}$ \\
\hline & Disconfirmed, neg & 0.881 & $22.70^{*}$ & 0.893 & $20.42^{*}$ & 0.882 & $25.08^{*}$ \\
\hline
\end{tabular}

Note: $\mathbf{A V E}$ = average variance extracted (average amount of variance of indicators that is explained by their respective latent variable); pos = positive scenario; neg = negative scenario; oth = other; sit = situation. Unconfirmed attributions are the initial ratings provided following the first statement presented; confirmed attributions are the final ratings provided when the respective attributional locus was supported by the preceding evidence; disconfirmed attributions are the final ratings provided when the respective attributional locus was not supported by the evidence. Supported = confirmed attributions; Unsupported = unconfirmed and disconfirmed attributions; $\mathbf{C R}=$ bootstrap critical ratio. The overall FIT $=.545$.

$\mathrm{CR}^{*}=$ significant at .05 level. 
Table 3.6. Component loadings for each latent variable from constrained multi-group GSCA.

\begin{tabular}{llcc}
\hline Latent Variable & Indicator & Loading & CR \\
\hline Supported & Self, pos & 0.766 & $10.61^{*}$ \\
AVE $=0.614$ & Self, neg & 0.749 & $14.52^{*}$ \\
& Other, pos & 0.829 & $23.78^{*}$ \\
& Other, neg & 0.876 & $33.75^{*}$ \\
& Situation, pos & 0.724 & $9.81^{*}$ \\
& Situation, neg & 0.745 & $15.65^{*}$ \\
Self - Unsupported & Unconfirmed, pos & & \\
AVE $=0.596$ & Unconfirmed, neg & 0.670 & $10.60^{*}$ \\
& Disconfirmed, pos & 0.850 & $32.59^{*}$ \\
& Disconfirmed, neg & 0.812 & $21.48^{*}$ \\
Other - Unsupported & Unconfirmed, pos & 0.743 & $14.34^{*}$ \\
AVE $=0.655$ & Unconfirmed, neg & 0.845 & $37.76^{*}$ \\
& Disconfirmed, pos & 0.818 & $27.23^{*}$ \\
& Disconfirmed, neg & 0.824 & $27.06^{*}$ \\
Situation - Unsupported & Unconfirmed, pos & 0.745 & $16.49^{*}$ \\
AVE $=0.816$ & Unconfirmed, neg & 0.921 & $68.42^{*}$ \\
& Disconfirmed, pos & 0.901 & $49.15^{*}$ \\
& Disconfirmed, neg & 0.907 & $60.61^{*}$ \\
\hline
\end{tabular}

Note: $\mathbf{A V E}$ = average variance extracted (average amount of variance of indicators that is explained by their respective latent variable); $\mathbf{p o s}=$ positive scenario; $\mathbf{n e g}=$ negative scenario. Unconfirmed attributions are the initial ratings provided following the first statement presented; confirmed attributions are the final ratings provided when the respective attributional locus was supported by the preceding evidence; disconfirmed attributions are the final ratings provided when the respective attributional locus was not supported by the evidence. Supported = confirmed attributions; Unsupported $=$ unconfirmed and disconfirmed attributions. $\mathbf{C R}=$ bootstrap critical ratio. The overall FIT $=.543$.

$\mathrm{CR}^{*}=$ significant at .05 level.

\subsubsection{Task performance}

For all analyses involving group contrasts, attribution ratings were first regressed onto the potential confounds that were found in Section 3.4.1. (IQ, years of education, age, and SES), and so the following analyses were employed on the re-standardized residuals obtained from this procedure. This resulted in loss of data for four participants, reducing the sample size to $n=146$ 
( $n=58$ healthy participants, $n=41$ schizophrenia patients, and $n=47$ bipolar patients). Analyses of the whole sample $(n=150)$ were run on the observed ASB data rather than residuals.

\section{Initial attribution ratings}

Percentage of variance explained. IQ, years of education, age, and SES combined accounted for $2.76 \%$ of the total variance in initial attribution ratings. With these confounds partialled out of $Z$, differences between schizophrenia patients, bipolar disorder patients, and healthy individuals only accounted for $1.29 \%$ of the remaining variance in initial attribution ratings. With $G$ coding paranoid schizophrenia, non-paranoid schizophrenia, bipolar disorder, and healthy participant groups, GC still accounted for very little variance (1.87\%). Finally, depressed, non-depressed, and healthy participant groupings accounted for only $1.21 \%$ of the variance in $Z$. Therefore, none of the internal analyses (i.e., PCA on the $G C$ and $E$ matrices) were performed.

Repeated measures ANOVA. To examine mean initial attribution ratings, a 2 (positive/negative scenario) $\times 3$ (self/other/situation rating) repeated measures ANOVA was performed on the whole sample. Group effects were not examined, as the above analysis demonstrated that group differences account for a negligible amount of variance in initial attribution ratings. However, there were within-subject effects for scenario, $F(1,147)=31.29, p$ $<.001$, and attribution, $F(2,262.72)=12.04, p<.001$, as well as a scenario $\times$ attribution interaction, $F(1.67,245.52)=297.49, p<.001$.

To examine these effects, paired t-tests were performed to compare the ratings between positive and negative scenarios for each attribution. Self attribution ratings for positive scenarios were significantly higher than those for negative scenarios (mean positive $=6.73, S D=1.95$; mean negative $=3.62, S D=1.80 ;$ paired sample $r=.46, p<.001 ; t(149)=19.59, p<.001)$. 
Other-person attribution ratings for positive scenarios were significantly lower than those for negative scenarios (mean positive $=3.95, S D=1.84$; mean negative $=6.01, S D=2.01$; paired sample $r=.64, p<.001 ; t(149)=15.27, p<.001)$. Situation attribution ratings did not differ between positive and negative scenarios (mean positive $=4.49, S D=2.36$; mean negative $=4.41$, $S D=2.20$; paired samples $r=.79, p<.001 ; t(149)=0.65, p=.515)$. These relationships are presented in Figure 3.1. below.

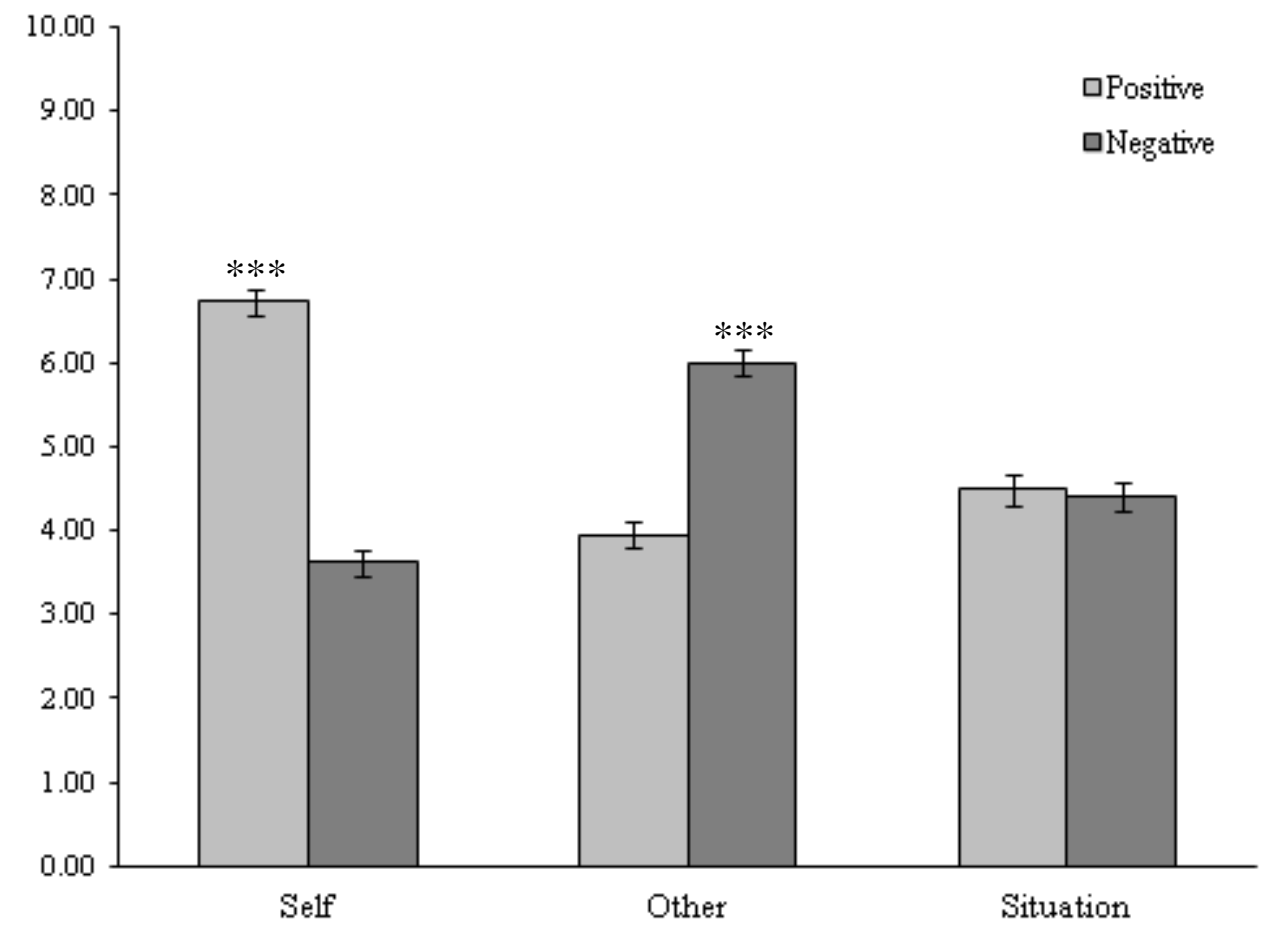

Figure 3.1. Mean initial attribution ratings for positive and negative scenarios (whole sample, $n=150)$. ***p $<.001$.

\section{All attribution ratings}

The series of regressions performed on initial attribution ratings was repeated with initial and final attribution ratings all entered into $Z$. IQ, years of education, age, and SES combined accounted for $4.13 \%$ of the total variance in attribution ratings. With these confounds partialled out of $Z$, differences between schizophrenia patients, bipolar disorder patients, and healthy 
individuals accounted for $1.33 \%$ of the remaining variance. With healthy, paranoid, nonparanoid, and bipolar groups coded in $G$, group differences accounted for $2.36 \%$ of the variance. Finally, healthy, depressed, and non-depressed groupings accounted for $1.57 \%$ of the variance. Again, none of the internal analyses were performed.

As a final exploration of this issue, a multivariate ANOVA (MANOVA) was performed with the four latent variables obtained from GSCA entered as dependent variables (i.e., supported attributions, unsupported self attributions, unsupported other-person attributions, and unsupported situation attributions; computed using component weights obtained from constrained GSCA), and group membership entered as a fixed factor (i.e., contrasts between healthy participants, schizophrenia patients, and bipolar disorder participants). As expected, this analysis did not reveal a significant main effect of group, $\Lambda_{\text {Wilks }}=.920, F(8,288)=1.53, p>.10$, $\eta^{2}=.041$

\subsection{Summary of Study 1}

A four-component structure was obtained from performing PCA on all attribution ratings. Three of these components reflected common variance among unsupported attributions (i.e., unconfirmed and disconfirmed attributions), which was distinct for each of the three attributional loci. In addition, a "supported" attributions component included high contributions from all attribution ratings that were provided after receiving confirming evidence (i.e., confirmed attributions). Results from GSCA supported this conceptualization of the ASB task. Further, the comparison of FIT between unconstrained and constrained models suggested that this model is appropriate for each of the three diagnostic groups (healthy individuals, schizophrenia patients, and bipolar disorder patients). 
Group differences accounted for a negligible amount of variance in responses on the ASB task, as displayed in attribution ratings and latent variable scores obtained from GSCA. However, the sample as a whole displayed a self-serving and personalizing bias reflected in mean unconfirmed attributions, with self attributions for positive scenarios and other-person attributions for negative scenarios being rated exceedingly high. There was no difference between positive scenarios and negative scenarios for situation attributions, which were generally rated low (but higher than self attributions for negative scenarios and other-person attributions for positive scenarios). 


\section{Chapter 4: Symptom-Related Attributional Biases (Study 2)}

\subsection{Aims}

Study 1 did not demonstrate significant differences between schizophrenia patients, bipolar disorder patients, and healthy control groups, even when patients were further divided into symptom-based subgroups. A promising alternative to studying differences between diagnostic categories is to examine a range of symptoms that cross nosological boundaries (Blom \& Van Praag, 2011; Murray, 2011). Given the heterogeneity of symptoms in schizophrenia and bipolar disorder, a multivariate analysis including a wide range of symptoms as a predictor may be more informative than diagnostic categories or groupings based on an individual symptom score. Further, the presence of subclinical psychopathology in the healthy population presents an opportunity to examine effects of schizotypical traits. The following study addresses Objective no. 3 (described in Chapter 1); that is, to examine attributional biases that can be explained by psychotic symptoms in schizophrenia and bipolar disorder patients, as well as schizotypical traits in healthy participants. Specifically, the following analyses aimed to identify components of the ASB task that can be predicted by psychotic symptoms and schizotypical traits, and whether these relationships remain after accounting for general neurocognitive demands and response perseveration in the ASB task.

\subsection{Predictions}

Study 1 demonstrated that four components account for the majority of variance in the ASB task, three of which are driven by attributions lacking supportive evidence (i.e., unconfirmed and disconfirmed attributions). However, the contributions on these components were fairly broad, with each component encompassing all unsupported attributions for the respective attributional locus, whether their ratings were for positive or negative scenarios. In the 
present study, it was expected that more specific patterns of intercorrelations would emerge from the variance in attributions that is predictable from psychotic symptoms/schizotypical traits. For example, it is plausible that a component dominated by high negative loadings for confirmed self attributions and high positive loadings for unsupported other-person attributions for negative scenarios (or some similar component(s) reflecting self-serving and/or personalizing biases) would emerge from the predicted solution and would be highly correlated with paranoid delusions (in patients). Depression was not expected to be correlated with a BADE effect (i.e., no involvement of disconfirmed attributions). However, given the lack of significant differences between groups when paranoia and depression were implicated in the symptom-based participant groupings in Study 1, these predictions were fairly tentative.

\subsection{Methods}

\subsubsection{Participants}

\section{Patients}

As demonstrated in the previous chapter, there were no significant differences between bipolar disorder patients and schizophrenia patients on demographic measures of interest or the ASB task. Therefore, the two patient groups were combined to increase power for the following analyses. In addition, some exclusion criteria (i.e., substance use, history of head injury, comorbid Axis I diagnosis) were relaxed to include a more heterogeneous sample of patients with psychotic illness. A total of $n=95$ patients were included in the following analyses (48 bipolar patients, 47 schizophrenia patients); see Table 4.1. for demographic information and IQ.

\section{Nonclinical participants}

The exclusion criteria described in Section 2.1. were relaxed so that healthy individuals with a previous personal and/or family history of psychiatric diagnosis could be included in the 
following analyses. This was to allow greater variance in CAPE symptom dimension scores, as apparently healthy individuals with a psychiatric history may be more likely to report higher occurrences of symptomatic experiences, and excluding these participants would negate the use of this symptom measure. A total of $n=69$ healthy participants were included in the following analyses; see Table 4.1. below for demographic information and IQ.

Table 4.1. Study 2 sample means and standard deviations (unless otherwise specified) for demographic variables and IQ.

\begin{tabular}{lcc}
\hline Variables & Patients $(n=95)$ & Nonclinical $(n=69)$ \\
\hline Gender $(\mathrm{m} / \mathrm{f})$ & $49 / 46$ & $38 / 31$ \\
KBIT IQ & $99.61(12.84)$ & $106.13(10.98)$ \\
Years of education & $13.72(2.37)$ & $15.71(2.20)$ \\
Years of age & $35.86(10.30)$ & $30.22(9.40)$ \\
SES & $72.62(24.54)$ & $62.97(18.57)$ \\
\hline
\end{tabular}

\subsubsection{Symptom measures}

\section{Signs and Symptoms of Psychotic Illness (SSPI)}

Full details on administration of the SSPI are provided in Section 2.2.2. In brief, the SSPI consists of 20 symptom items, with additional subscales for content of delusions and hallucinations. Each symptom and its subscales (if applicable) are rated on a 5-point scale, ranging from 0 (not present) to 4 (severe). In a preliminary CPCA (with $Z=$ ASB and $G=$ SSPI), all SSPI symptom and subscale scores were entered into $G$. The only symptom subscale with reasonably high component loadings in these preliminary analyses was paranoid delusions; therefore, $G$ was comprised of all general symptom scores and the paranoid subscale for delusions in the following analyses, for a total of 21 variables. Mean SSPI scores for all patients are listed in Table 4.2. below. 
Table 4.2. Study 2 SSPI symptom scores for all patients $(\mathrm{n}=95)$.

\begin{tabular}{lc}
\hline Symptoms & Mean $(S D)$ \\
\hline Anxiety & $1.27(1.06)$ \\
Depression & $1.07(1.22)$ \\
Anhedonia & $1.05(1.13)$ \\
Elated mood & $0.58(0.97)$ \\
Insomnia & $1.09(1.20)$ \\
Somatic complaints & $0.14(0.45)$ \\
Delusions - general & $1.47(1.44)$ \\
Delusions - guilt/worthlessness & $0.27(0.72)$ \\
Delusions - grandiose & $0.66(1.14)$ \\
Delusions - paranoid & $0.96(1.33)$ \\
Delusions - Schneiderian & $0.65(1.21)$ \\
Hallucinations - general & $1.00(1.61)$ \\
Hallucinations - 2 ${ }^{\text {nd }}$ person auditory & $0.71(1.47)$ \\
Hallucinations - Schneiderian & $0.62(1.36)$ \\
Impaired attention & $1.34(0.94)$ \\
Disorientation & $0.03(0.18)$ \\
Overactivity & $0.97(1.05)$ \\
Underactivity & $0.97(1.06)$ \\
Flattened affect & $0.93(0.99)$ \\
Inappropriate affect & $0.16(0.64)$ \\
Pressured speech & $0.36(0.77)$ \\
Poverty of speech & $0.25(0.60)$ \\
Disordered thought & $0.28(0.75)$ \\
Peculiar behaviour & $0.13(0.44)$ \\
Irritability/hostility & $0.42(0.69)$ \\
Impaired insight & $1.12(1.16)$ \\
Total (excluding subscales) & $14.63(7.58)$ \\
\hline
\end{tabular}

\section{Community Assessment of Psychic Experiences (CAPE)}

Full details on administration of the CAPE are described in Section 2.2.2. on page 22. In brief, the CAPE is a self-report questionnaire that measures how often an individual experiences given signs of psychosis, and how distressed s/he is by these experiences. The CAPE consists of forty such questions, which comprise three symptom dimensions: positive symptoms, negative symptoms, and depression. Each symptom dimension score is weighted such that the lowest possible score is 1.00 (responding "never" on all questions answered) and the highest possible 
score is 4.00 (responding "nearly always" on all questions answered). Mean CAPE symptom scores for all nonclinical participants were: (1) mean positive $=1.37, S D=0.29$; (2) mean negative $=1.72, S D=0.41 ;(3)$ mean depression $=1.65, S D=0.32$.

\subsubsection{Aim no. 1 (symptom-related attributional biases in patients)}

The first goal of this study was to determine the extent to which SSPI scores could account for responses on the ASB task, and to examine the components of the ASB task that are predictable from these symptoms. Similar to Study 1, CPCA was first run with only the initial (unconfirmed) attribution ratings entered into $Z$, and with SSPI symptoms entered into $G$. The second CPCA was run with the same $G$ matrix, but with all attribution ratings included in $Z$. To determine whether relationships between attribution ratings and symptoms would change after partialling general neurocognitive function out of the ASB task, the residuals obtained from regressing all attribution ratings onto standard neurocognitive measures (KBIT-II verbal and non-verbal IQ, and WCST percent perseverative errors; Section 2.2.1.) were re-standardized and

entered into $Z$ for the third CPCA (again with SSPI scores in $G$ ). Unconstrained PCAs of the $Z$ matrices were not performed, as the component structure of the ASB task was investigated extensively in Study 1.

\subsubsection{Aim no. 2 (symptom-related attributional biases in nonclinical participants)}

The procedures for the analysis in nonclinical participants were virtually identical to those in patients described above, except that the CAPE symptom dimensions were entered in $G$ rather than SSPI scores (which were not available for healthy participants in this dataset). 


\subsection{Results}

\subsubsection{Patients}

\section{CPCA no. 1: Initial attribution ratings and SSPI}

Table 4.3. below presents the distribution of variance among the elements of CPCA in patients, with initial attribution ratings entered in $Z$ and SSPI scores entered in $G$. One component was extracted from each source of variance obtained from the external analysis.

Table 4.3. Distribution of variance for CPCA no. 1 in patients $(\mathrm{Z}=$ initial attribution ratings, $\mathrm{G}=$ SSPI).

\begin{tabular}{ccc}
\hline Portion of Variance & External & $\begin{array}{c}\text { Internal } \\
(1 \text { component })\end{array}$ \\
\hline Predicted $(G C-$ SSPI $)$ & 1.65 & 1.07 \\
\% Predicted & $\mathbf{1 0 0 . 0 0}$ & $\mathbf{6 4 . 6 5}$ \\
\% Total & $\mathbf{2 7 . 5 2}$ & $\mathbf{1 7 . 7 9}$ \\
\hline Residual $(E)$ & 4.35 & 2.69 \\
\% Residual & $\mathbf{1 0 0 . 0 0}$ & $\mathbf{6 1 . 8 0}$ \\
\% Total & $\mathbf{7 2 . 4 8}$ & $\mathbf{4 4 . 7 9}$ \\
\hline
\end{tabular}

Note: The variances accounted for from the external analysis (regression) and by the components extracted from the internal analysis (PCA) are listed in regular font. The percentages of variance accounted for by each source are listed in bold font. \% Total = percentage of total variance explained by the source identified in each column. $\%$ Predicted $=$ percentage of predictable variance explained by the source identified in each column. \% Residual $=$ percentage of residual variance explained by the source identified in each column.

Analysis of $\boldsymbol{G C}$ and $\boldsymbol{G}$. PCA of the SSPI-predicted variance revealed a one-component solution in $G C$, which was dominated by self attributions for negative scenarios and situation attributions for positive and negative scenarios. This component was most highly correlated with overactivity, disordered thought, inappropriate affect, and, to a lesser degree, elated mood, underactivity (loaded negatively), disorientation, peculiar behavior, and pressured speech (as suggested by $G C$ component loadings and $G$ loadings, listed in Table 4.4.).

Internal analysis of $\boldsymbol{E}$. PCA of the residual variance in initial attribution ratings also revealed a one-component solution. This component consisted of high contributions from all 
attributions, with the highest contribution from situation attributions on negative scenarios (.732) and the lowest from self attributions on positive scenarios (.581), as listed in Table 4.5.

Table 4.4. GC component loadings and $\mathrm{G}$ loadings from CPCA no. 1 in patients $(\mathrm{Z}=$ initial attribution ratings, $\mathrm{G}=S S P I$ ).

\begin{tabular}{lcclc}
\hline \multicolumn{2}{c}{$G C$} & & & \multicolumn{2}{c}{$G$} \\
\cline { 1 - 1 } Attributions & Loadings & & Symptoms & Loadings \\
\cline { 1 - 2 } Self, neg & $\mathbf{0 . 5 6 0}$ & & Overactivity & $\mathbf{0 . 5 6 4}$ \\
Situation, neg & $\mathbf{0 . 5 2 1}$ & & Disordered thought & $\mathbf{0 . 4 8 1}$ \\
Situation, pos & $\mathbf{0 . 4 6 4}$ & & Inappropriate Affect & $\mathbf{0 . 4 1 7}$ \\
Other, pos & 0.333 & & Elated mood & 0.396 \\
Self, pos & 0.300 & & Underactivity & -0.392 \\
Other, neg & 0.256 & & Disorientation & 0.386 \\
& & & Peculiar behaviour & 0.385 \\
& & Pressured speech & 0.377 \\
& & Delusions - general & 0.322 \\
& & & Delusions - paranoid & 0.302 \\
& & Attentional impairment & 0.268 \\
& & Hallucinations - general & 0.262 \\
& & Irritability/hostility & 0.188 \\
& & Impaired insight & 0.186 \\
& & Insomnia & -0.172 \\
& & Anhedonia & -0.137 \\
& & Anxiety & 0.119 \\
& & Flattened affect & -0.079 \\
& & Poverty of speech & 0.075 \\
& & Somatic complaints & -0.062 \\
& & Depression & -0.033 \\
\hline
\end{tabular}

Note: variables sorted by absolute loadings (descending). Loadings $\geq .40$ are set in bold font. neg = negative scenario; pos = positive scenario.

Table 4.5. E component loadings from CPCA no. 1 in patients $(\mathrm{Z}=$ initial attribution ratings, $\mathrm{G}=$ SSPI).

\begin{tabular}{lc}
\hline Attributions & Loadings \\
\hline Self, pos & 0.718 \\
Self, neg & 0.581 \\
Other, pos & 0.653 \\
Other, neg & 0.682 \\
Situation, pos & 0.638 \\
Situation, neg & 0.732 \\
\hline
\end{tabular}

Note: $\mathbf{p o s}=$ positive scenario; $\mathbf{n e g}=$ negative scenario . 


\section{CPCA no. 2: All attribution ratings and SSPI}

Table 4.6. below presents the distribution of variance among the elements of CPCA, with all observed attribution ratings entered in $Z$. Two components were extracted from each source of variance obtained from the external analysis.

Table 4.6. Distribution of variance for CPCA no. 2 in patients $(\mathrm{Z}=$ all observed attribution ratings, $\mathrm{G}=S S P I)$.

\begin{tabular}{ccccc}
\hline & & \multicolumn{3}{c}{ Internal } \\
\cline { 3 - 4 } Portion of Variance & External & 1 & 2 & All \\
\hline Predicted $(G C-$ SSPI $)$ & 4.54 & 1.78 & 1.30 & 3.08 \\
\% Predicted & $\mathbf{1 0 0 . 0 0}$ & $\mathbf{3 9 . 2 0}$ & $\mathbf{2 8 . 5 9}$ & $\mathbf{6 7 . 7 9}$ \\
\% Total & $\mathbf{2 5 . 2 1}$ & $\mathbf{9 . 8 8}$ & $\mathbf{7 . 2 1}$ & $\mathbf{1 7 . 0 9}$ \\
\hline Residual $(E)$ & 13.46 & 4.35 & 3.63 & 7.98 \\
\% Residual & $\mathbf{1 0 0 . 0 0}$ & $\mathbf{3 2 . 2 9}$ & $\mathbf{2 6 . 9 8}$ & $\mathbf{5 9 . 2 7}$ \\
\% Total & $\mathbf{7 4 . 7 9}$ & $\mathbf{2 4 . 1 5}$ & $\mathbf{2 0 . 1 8}$ & $\mathbf{4 4 . 3 3}$ \\
\hline
\end{tabular}

Note: The variances accounted for from the external analysis (regression) and by the components extracted from the internal analysis (PCA) are listed in regular font. The percentages of variance accounted for by each source are listed in bold font. \% Total = percentage of total variance explained by the source identified in each column. \% Predicted $=$ percentage of predictable variance explained by the source identified in each column. $\%$ Residual $=$ percentage of residual variance explained by the source identified in each column. Components are ordered by decreasing magnitude of total variance explained.

Analysis of $\boldsymbol{G C}$ and $\boldsymbol{G}$. PCA of the SSPI-predicted variance revealed a two-component solution in $G C$, dominated by: (1) unsupported self attributions for negative scenarios, as well as unsupported situation attributions for positive and negative scenarios, and (2) most other-person attributions. Component 1 was highly correlated with inappropriate affect, disordered thought, overactivity and, to a lesser extent, paranoid delusions, attentional impairment, disorientation, and peculiar behaviour. Component 2 was positively correlated with depression and anxiety, and, to a lesser extent, negatively correlated with poverty of speech (suggested by $G C$ component loadings and $G$ loadings, listed in Table 4.7.).

Internal analysis of $\boldsymbol{E}$. PCA on the residual variance revealed a two-component solution, dominated by: (1) all unsupported attributions (except unconfirmed other-person attributions on 
negative scenarios, which had a higher contribution on component 2), and (2) all confirmed attributions (although unconfirmed self attributions for positive scenarios also had a reasonably high loading at .457 , its contribution was higher on component 1 , at .543$)$. E component loadings are listed in Table 4.8.

Table 4.7. GC component loadings and $\mathrm{G}$ loadings from CPCA no. 2 in patients $(\mathrm{Z}=$ all observed attribution ratings, $\mathrm{G}=S S P I)$.

\begin{tabular}{|c|c|c|c|c|c|}
\hline \multicolumn{3}{|c|}{$G C$} & \multicolumn{3}{|c|}{$G$} \\
\hline Attributions & 1 & 2 & Symptoms & 1 & 2 \\
\hline Self unconfirmed, neg & 0.560 & 0.119 & Inappropriate affect & 0.566 & -0.032 \\
\hline Sit disconfirmed, neg & 0.538 & 0.172 & Disordered thought & 0.517 & 0.096 \\
\hline Sit unconfirmed, neg & 0.508 & 0.130 & Overactivity & 0.489 & 0.171 \\
\hline Sit unconfirmed, pos & 0.494 & 0.060 & Delusions - paranoid & 0.387 & 0.035 \\
\hline Self disconfirmed, neg & 0.482 & -0.022 & Attentional impairment & 0.370 & -0.132 \\
\hline Sit disconfirmed, pos & 0.480 & 0.115 & Disorientation & 0.358 & 0.118 \\
\hline Oth disconfirmed, pos & 0.087 & 0.417 & Peculiar behaviour & 0.354 & 0.159 \\
\hline Oth unconfirmed, neg & 0.088 & 0.417 & Depression & -0.221 & 0.479 \\
\hline Oth confirmed, neg & -0.013 & 0.416 & Anxiety & -0.097 & 0.449 \\
\hline Oth unconfirmed, pos & 0.152 & 0.393 & Poverty of speech & 0.234 & -0.390 \\
\hline Oth confirmed, pos & -0.055 & 0.350 & Elated mood & 0.252 & 0.354 \\
\hline Self unconfirmed, pos & 0.235 & 0.128 & Delusions - general & 0.342 & 0.036 \\
\hline Self disconfirmed, pos & 0.218 & 0.019 & Pressured speech & 0.301 & 0.234 \\
\hline Sit confirmed, pos & 0.153 & 0.336 & Underactivity & -0.275 & -0.278 \\
\hline Oth disconfirmed, neg & 0.141 & 0.246 & Insomnia & -0.273 & 0.150 \\
\hline Sit confirmed, neg & 0.122 & 0.224 & Hallucinations & 0.233 & 0.138 \\
\hline Self confirmed, pos & 0.091 & 0.303 & Impaired insight & 0.205 & -0.032 \\
\hline \multirow[t]{4}{*}{ Self confirmed, neg } & 0.021 & 0.298 & Anhedonia & -0.147 & 0.005 \\
\hline & & & Irritability/hostility & 0.055 & 0.220 \\
\hline & & & Somatic complaints & -0.042 & -0.164 \\
\hline & & & Flattened affect & 0.030 & -0.220 \\
\hline
\end{tabular}

Note: variables are sorted by absolute loadings (descending). Loadings $\geq .40$ are set in bold font. Components are ordered by decreasing magnitude of total variance explained. pos = positive scenario; neg $=$ negative scenario; oth $=$ other, sit $=$ situation. Unconfirmed attributions are the initial ratings provided following the first statement presented; confirmed attributions are the final ratings provided when the respective attributional locus was supported by the preceding evidence; disconfirmed attributions are the final ratings provided when the respective attributional locus was not supported by the evidence. 
Table 4.8. E component loadings from CPCA no. 2 in patients $(\mathrm{Z}=$ all observed attribution ratings, $\mathrm{G}=$ SSPI).

\begin{tabular}{lcc}
\hline Attributions & 1 & 2 \\
\hline Sit unconfirmed, pos & $\mathbf{0 . 6 6 9}$ & 0.111 \\
Sit unconfirmed, neg & $\mathbf{0 . 6 5 7}$ & 0.282 \\
Self disconfirmed, pos & $\mathbf{0 . 6 4 0}$ & 0.123 \\
Sit disconfirmed, neg & $\mathbf{0 . 6 3 5}$ & -0.020 \\
Oth disconfirmed, neg & $\mathbf{0 . 6 1 7}$ & 0.055 \\
Sit disconfirmed, pos & $\mathbf{0 . 5 9 7}$ & 0.100 \\
Self disconfirmed, neg & $\mathbf{0 . 5 9 0}$ & -0.191 \\
Self unconfirmed, neg & $\mathbf{0 . 5 7 2}$ & 0.099 \\
Oth disconfirmed, pos & $\mathbf{0 . 5 7 1}$ & 0.179 \\
Self unconfirmed, pos & $\mathbf{0 . 5 4 3}$ & $\mathbf{0 . 4 5 7}$ \\
Oth unconfirmed, pos & $\mathbf{0 . 5 3 2}$ & 0.208 \\
Oth confirmed, neg & -0.010 & $\mathbf{0 . 7 7 7}$ \\
Oth confirmed, pos & 0.100 & $\mathbf{0 . 7 7 3}$ \\
Self confirmed, neg & -0.073 & $\mathbf{0 . 6 8 7}$ \\
Sit confirmed, neg & 0.009 & $\mathbf{0 . 6 8 7}$ \\
Self confirmed, pos & 0.284 & $\mathbf{0 . 6 3 1}$ \\
Sit confirmed, pos & 0.291 & $\mathbf{0 . 5 7 5}$ \\
Oth unconfirmed, neg & 0.395 & $\mathbf{0 . 5 5 5}$ \\
\hline
\end{tabular}

Note: variables are sorted by absolute loadings (descending). Loadings $\geq .40$ are set in bold font. Components are ordered by decreasing magnitude of total variance explained. pos $=$ positive scenario; neg = negative scenario; oth $=$ other; sit $=$ situation. Unconfirmed attributions are the initial ratings provided following the first statement presented; confirmed attributions are the final ratings provided when the respective attributional locus was supported by the preceding evidence; disconfirmed attributions are the final ratings provided when the respective attributional locus was not supported by the evidence.

\section{CPCA no. 3: Neurocognitive measures partialled out of the ASB task}

Table 4.9. presents the distribution of variance among the elements of CPCA, after partialling out neurocognitive measures (KBIT verbal and nonverbal IQ, WCST percent perseverative errors) from all attribution ratings. These neurocognitive measures accounted for $6.30 \%$ of the total variance in attribution ratings. 
Table 4.9. Distribution of variance for CPCA no. 3 in patients $(\mathrm{Z}=$ all attribution ratings with neurocognitive measures partialled out, $\mathrm{G}=$ SSPI).

\begin{tabular}{ccccc}
\hline & & \multicolumn{3}{c}{ Internal } \\
\cline { 3 - 5 } Portion of Variance & External & 1 & 2 & All \\
\hline Predicted $(G C-S S P I)$ & 4.22 & 2.06 & - & 2.06 \\
\% Predicted & $\mathbf{1 0 0 . 0 0}$ & $\mathbf{4 8 . 7 9}$ & - & $\mathbf{4 8 . 7 9}$ \\
\% Total & $\mathbf{2 3 . 4 4}$ & $\mathbf{1 1 . 4 4}$ & - & $\mathbf{1 1 . 4 4}$ \\
\hline Residual $(E)$ & 13.78 & 4.63 & 3.52 & 8.15 \\
\% Predicted & $\mathbf{1 0 0 . 0 0}$ & $\mathbf{3 3 . 5 8}$ & $\mathbf{2 5 . 5 7}$ & $\mathbf{5 9 . 1 4}$ \\
\% Total & $\mathbf{7 6 . 5 6}$ & $\mathbf{2 5 . 7 1}$ & $\mathbf{1 9 . 5 7}$ & $\mathbf{4 5 . 2 8}$ \\
\hline
\end{tabular}

Note: The variances accounted for from the external analysis (regression) and by the components extracted from the internal analysis (PCA) are listed in regular font. The percentages of variance accounted for by each source are listed in bold font. \% Total $=$ percentage of total variance explained by the source identified in each column. \% Predicted $=$ percentage of predictable variance explained by the source identified in each column. $\%$ Residual $=$ percentage of residual variance explained by the source identified in each column. Components are ordered by decreasing magnitude of total variance explained.

Analysis of $\boldsymbol{G C}$ and $\boldsymbol{G}$. PCA revealed a one-component solution in $G C$. This component resembled component 1 of the predicted solution from CPCA no. 2, being dominated by unsupported situation attributions for all scenarios, unconfirmed self attributions for negative scenarios, and to a lesser extent, disconfirmed self attributions for negative scenarios. This component was highly correlated with overactivity, disordered thought, inappropriate affect, elated mood, and, to a lesser extent, peculiar behaviour and pressure of speech. $G C$ component loadings and $G$ loadings are listed in Table 4.10.

Internal analysis of $\boldsymbol{E}$. PCA on the residual variance revealed a two-component solution in E. These components were dominated by: (1) all unsupported attributions, and (2) all confirmed attributions (though, again, unconfirmed self attributions for positive scenarios and unconfirmed other-person attributions for negative scenarios showed reasonably high contributions on both components). $E$ component loadings are listed in Table 4.11. 
Table 4.10. GC component loadings and $\mathrm{G}$ loadings from CPCA no. 3 in patients $(\mathrm{Z}=$ all attribution ratings with neurocognitive measures partialled out, $\mathrm{G}=S S P I$ ).

\begin{tabular}{lcclrr}
\hline \multicolumn{1}{c}{$G C$} & & & \multicolumn{2}{c}{$G$} \\
\cline { 1 - 2 } \cline { 5 - 6 } Attributions & Loadings & & & Symptoms & Loadings \\
\cline { 1 - 2 } Sit disconfirmed, neg & $\mathbf{0 . 5 5 3}$ & & & Overactivity & $\mathbf{0 . 4 8 0}$ \\
Self unconfirmed, neg & $\mathbf{0 . 4 8 8}$ & & Disordered thought & $\mathbf{0 . 4 7 4}$ \\
Sit unconfirmed, neg & $\mathbf{0 . 4 7 2}$ & & Inappropriate affect & $\mathbf{0 . 4 2 5}$ \\
Sit disconfirmed, pos & $\mathbf{0 . 4 5 2}$ & & Elated mood & $\mathbf{0 . 4 2 2}$ \\
Sit unconfirmed, pos & $\mathbf{0 . 4 1 7}$ & & Peculiar behaviour & 0.394 \\
Self disconfirmed, neg & 0.380 & & Pressured speech & 0.392 \\
Oth unconfirmed, pos & 0.349 & & Underactivity & -0.371 \\
Oth disconfirmed, pos & 0.323 & & Disorientation & 0.344 \\
Sit confirmed, pos & 0.321 & & Delusions - paranoid & 0.281 \\
Oth unconfirmed, neg & 0.304 & & Delusions - general & 0.245 \\
Oth disconfirmed, neg & 0.268 & & Hallucinations & 0.205 \\
Oth confirmed, neg & 0.240 & & Attentional impairment & 0.191 \\
Self unconfirmed, pos & 0.218 & & Anxiety & 0.172 \\
Sit confirmed, neg & 0.218 & & Irritability/hostility & 0.165 \\
Self confirmed, pos & 0.208 & & Somatic complaints & -0.163 \\
Self confirmed, neg & 0.197 & & Anhedonia & -0.140 \\
Self disconfirmed, pos & 0.148 & & Flattened affect & -0.132 \\
Oth confirmed, pos & 0.141 & & Insomnia & -0.131 \\
& & & Depression & 0.128 \\
& & & Impaired insight & 0.096 \\
& & & Poverty of speech & -0.032 \\
\hline
\end{tabular}

Note: variables are sorted by absolute loadings (descending). Loadings $\geq .40$ are set in bold font. pos $=$ positive scenario; neg $=$ negative scenario; oth $=$ other; sit $=$ situation . Unconfirmed attributions are the initial ratings provided following the first statement presented; confirmed attributions are the final ratings provided when the respective attributional locus was supported by the preceding evidence; disconfirmed attributions are the final ratings provided when the respective attributional locus was not supported by the evidence. 
Table 4.11. E component loadings from CPCA no. 3 in patients $(\mathrm{Z}=$ all attribution ratings with neurocognitive measures partialled out, $\mathrm{G}=$ SSPI).

\begin{tabular}{lcc}
\hline Attributions & 1 & 2 \\
\hline Sit unconfirmed, neg & $\mathbf{0 . 6 8 6}$ & 0.255 \\
Sit unconfirmed, pos & $\mathbf{0 . 6 7 6}$ & 0.130 \\
Sit disconfirmed, neg & $\mathbf{0 . 6 4 0}$ & 0.033 \\
Self disconfirmed, pos & $\mathbf{0 . 6 1 8}$ & 0.200 \\
Oth unconfirmed, pos & $\mathbf{0 . 6 1 3}$ & 0.111 \\
Self unconfirmed, neg & $\mathbf{0 . 6 1 0}$ & 0.082 \\
Oth disconfirmed, neg & $\mathbf{0 . 6 0 0}$ & 0.075 \\
Oth disconfirmed, pos & $\mathbf{0 . 5 9 0}$ & 0.152 \\
Self disconfirmed, neg & $\mathbf{0 . 5 9 0}$ & -0.105 \\
Sit disconfirmed, pos & $\mathbf{0 . 5 7 5}$ & 0.179 \\
Self unconfirmed, pos & $\mathbf{0 . 5 7 1}$ & $\mathbf{0 . 4 6 1}$ \\
Oth unconfirmed, neg & $\mathbf{0 . 4 6 4}$ & $\mathbf{0 . 4 9 7}$ \\
Oth confirmed, neg & 0.038 & $\mathbf{0 . 7 8 0}$ \\
Oth confirmed, pos & 0.128 & $\mathbf{0 . 7 6 5}$ \\
Sit confirmed, neg & 0.028 & $\mathbf{0 . 6 9 6}$ \\
Self confirmed, neg & -0.043 & $\mathbf{0 . 6 8 2}$ \\
Self confirmed, pos & 0.318 & $\mathbf{0 . 6 2 0}$ \\
Sit confirmed, pos & 0.334 & $\mathbf{0 . 5 6 9}$ \\
\hline
\end{tabular}

Note: variables are sorted by absolute loadings (descending). Loadings $\geq .40$ are set in bold font. Components are ordered by decreasing magnitude of total variance explained. pos $=$ positive scenario; neg $=$ negative scenario; oth $=$ other; sit $=$ situation. Unconfirmed attributions are the initial ratings provided following the first statement presented; confirmed attributions are the final ratings provided when the respective attributional locus was supported by the preceding evidence; disconfirmed attributions are the final ratings provided when the respective attributional locus was not supported by the evidence.

To summarize the results observed in patients, CPCAs revealed a general pattern of shared variance among unsupported (i.e., unconfirmed and disconfirmed) self-blame and situation attributions in the SSPI-predicted solutions, which appeared to be highly correlated with symptoms of disorganization and mania. Although a second "other-person" component emerged from the predicted solution in CPCA no. 2 (which was most highly correlated with depression and anxiety), this component did not remain after removing variance due to neurocognitive demands from the ASB task. 


\subsubsection{Nonclinical participants}

\section{CPCA no. 1: Initial attribution ratings and CAPE}

Table 4.12. below presents the distribution of variance among the elements of CPCA in nonclinical participants, with initial attribution ratings entered in $Z$ and CAPE symptom scores in $G$. One component was extracted from each source of variance obtained from the external analysis.

Table 4.12. Distribution of variance for CPCA no. 1 in nonclinical participants $(\mathrm{Z}=$ initial attribution ratings, $\mathrm{G}=C A P E)$.

\begin{tabular}{ccc}
\hline Portion of Variance & External & $\begin{array}{c}\text { Internal } \\
\text { (1 component })\end{array}$ \\
\hline Predicted $(G C-$ CAPE) & 0.66 & 0.47 \\
\% Predicted & $\mathbf{1 0 0 . 0 0}$ & $\mathbf{7 1 . 3 1}$ \\
\% Total & $\mathbf{1 0 . 9 4}$ & $\mathbf{7 . 8 0}$ \\
\hline Residual $(E)$ & 5.34 & 2.82 \\
\% Residual & $\mathbf{1 0 0 . 0 0}$ & $\mathbf{5 2 . 7 3}$ \\
\% Total & $\mathbf{8 9 . 0 6}$ & $\mathbf{4 6 . 9 6}$ \\
\hline
\end{tabular}

Note: The variances accounted for from the external analysis (regression) and by the components extracted from the internal analysis (PCA) are listed in regular font. The percentages of variance accounted for by each source are listed in bold font. \% Total $=$ percentage of total variance explained by the source identified in each column. \% Predicted $=$ percentage of predictable variance explained by the source identified in each column. $\%$ Residual $=$ percentage of residual variance explained by the source identified in each column.

Analysis of $\boldsymbol{G C}$ and $\boldsymbol{G}$. PCA of the CAPE-predicted variance revealed a one-component solution in $G C$. This component was dominated by self attributions for positive scenarios $(G C$ component loading $=.414)$, which was negatively correlated with frequency of depression $(r=$ -.861). See Table 4.13. for the full list of $G C$ component loadings and $G$ loadings.

Internal analysis of $\boldsymbol{E}$. PCA of the residual variance also revealed a one-component solution in $E$. All attributions displayed high positive loadings on this component, with the greatest contribution from other-person attributions for positive scenarios (.811) and the lowest from self attributions for positive scenarios (.565). See Table 4.14. for the list of all $E$ component loadings. 
Table 4.13. GC component loadings and $\mathrm{G}$ loadings from CPCA no. 1 in nonclinical participants $(\mathrm{Z}=$ initial attribution ratings, $\mathrm{G}=C A P E)$.

\begin{tabular}{|c|c|c|c|}
\hline \multicolumn{2}{|c|}{$G C$} & \multicolumn{2}{|l|}{$G$} \\
\hline Attributions & Loadings & Symptom dimensions & Loadings \\
\hline Self, pos & 0.414 & Depression & -0.861 \\
\hline Situation, neg & 0.317 & Negative & -0.192 \\
\hline Situation, pos & 0.280 & Positive & 0.151 \\
\hline Other, neg & 0.219 & & \\
\hline Self, neg & 0.201 & & \\
\hline Other, pos & 0.171 & & \\
\hline
\end{tabular}

Note: variables sorted by absolute loadings (descending). Loadings $\geq .40$ are set in bold font. neg $=$ negative scenario; pos = positive scenario .

Table 4.14. E component loadings from CPCA no. 1 in nonclinical participants $(\mathrm{Z}=$ initial attribution ratings, $\mathrm{G}=C A P E)$.

\begin{tabular}{lc}
\hline Attributions & Loadings \\
\hline Self, pos & 0.565 \\
Self, neg & 0.612 \\
Other, pos & 0.811 \\
Other, neg & 0.625 \\
Situation, pos & 0.757 \\
Situation, neg & 0.708 \\
\hline
\end{tabular}

Note $:$ pos = positive scenario; neg = negative scenario.

\section{CPCA no. 2: All attribution ratings and CAPE}

Table 4.15. below presents the distribution of variance among the elements of CPCA, with all observed attribution ratings entered in $Z$. 
Table 4.15. Distribution of variance for CPCA no. 2 in nonclinical participants $(\mathrm{Z}=$ all observed attribution ratings, $\mathrm{G}=C A P E)$.

\begin{tabular}{ccccccc}
\hline & & \multicolumn{5}{c}{ Internal } \\
\cline { 3 - 7 } Portion of Variance & External & 1 & 2 & 3 & 4 & All \\
\hline Predicted $(G C-$ CAPE) & 1.53 & 1.01 & - & - & - & 1.01 \\
\% Predicted & $\mathbf{1 0 0 . 0 0}$ & $\mathbf{6 6 . 4 1}$ & - & - & - & $\mathbf{6 6 . 4 1}$ \\
\% Total & $\mathbf{8 . 4 8}$ & $\mathbf{5 . 6 3}$ & - & - & - & $\mathbf{5 . 6 3}$ \\
\hline Residual $(E)$ & 16.47 & 3.69 & 3.68 & 3.39 & 2.03 & 12.78 \\
\% Residual & $\mathbf{1 0 0 . 0 0}$ & $\mathbf{2 2 . 3 9}$ & $\mathbf{2 2 . 3 2}$ & $\mathbf{2 0 . 6 0}$ & $\mathbf{1 2 . 2 9}$ & $\mathbf{7 7 . 6 0}$ \\
\% Total & $\mathbf{9 1 . 5 2}$ & $\mathbf{2 0 . 4 9}$ & $\mathbf{2 0 . 4 3}$ & $\mathbf{1 8 . 8 6}$ & $\mathbf{1 1 . 2 5}$ & $\mathbf{7 1 . 0 2}$ \\
\hline
\end{tabular}

Note: The variances accounted for from the external analysis (regression) and by the components extracted from the internal analysis (PCA) are listed in regular font. The percentages of variance accounted for by each source are listed in bold font. \% Total $=$ percentage of total variance explained by the source identified in each column. \% Predicted $=$ percentage of predictable variance explained by the source identified in each column. $\%$ Residual $=$ percentage of residual variance explained by the source identified in each column. Components are ordered by decreasing magnitude of total variance explained.

Analysis of $\boldsymbol{G C}$ and $\boldsymbol{G}$. PCA on the CAPE-predicted variance revealed a one-component solution in $G C$. Although the loadings were somewhat small, this component was dominated by unconfirmed self attributions for positive scenarios (.382), unconfirmed situation attributions for negative scenarios (.330), and disconfirmed situation attributions for positive scenarios (.309); this component was negatively correlated with frequency of depression $(r=-.808)$. All $G C$ component loadings and $G$ loadings are listed in Table 4.16.

Internal analysis of $\boldsymbol{E}$. PCA of the residual variance revealed a four-component solution in $E$. The highest contributions on each were: (1) confirmed attributions, (2) unsupported situation attributions, (3) unsupported other attributions, and (4) unsupported self attributions (except for unconfirmed self attributions for positive scenarios, which had higher loadings on components 1 and 3). This pattern is similar to the four-component structure observed in $Z$ in Study 1, though with less resemblance to a simple structure (i.e., any given variable having a high loading on a single component and near-zero loadings on the others, and any given component consisting of only a few variables with very high loadings, with the remaining variables all having near-zero loadings). $E$ component loadings are listed in Table 4.17. 
Table 4.16. GC component loadings and $\mathrm{G}$ loadings from CPCA no. 2 in nonclinical participants $(\mathrm{Z}=$ all observed attribution ratings, $\mathrm{G}=C A P E)$.

\begin{tabular}{|c|c|c|c|}
\hline \multicolumn{2}{|l|}{$G C$} & \multicolumn{2}{|l|}{$G$} \\
\hline Attributions & Loadings & Symptom Dimensions & Loadings \\
\hline Self unconfirmed, pos & 0.382 & Depression & -0.808 \\
\hline Sit unconfirmed, neg & 0.330 & Positive & 0.148 \\
\hline Sit disconfirmed, pos & 0.309 & Negative & -0.036 \\
\hline Sit confirmed, pos & 0.282 & & \\
\hline Sit disconfirmed, neg & 0.278 & & \\
\hline Self disconfirmed, pos & 0.273 & & \\
\hline Sit unconfirmed, pos & 0.270 & & \\
\hline Oth confirmed, neg & 0.231 & & \\
\hline Self unconfirmed, neg & 0.226 & & \\
\hline Oth unconfirmed, neg & 0.221 & & \\
\hline Oth disconfirmed, neg & 0.215 & & \\
\hline Oth confirmed, pos & 0.210 & & \\
\hline Sit confirmed, neg & 0.189 & & \\
\hline Oth unconfirmed, pos & 0.172 & & \\
\hline Self confirmed, pos & 0.152 & & \\
\hline Oth disconfirmed, pos & 0.110 & & \\
\hline Self confirmed, neg & -0.108 & & \\
\hline Self disconfirmed, neg & 0.054 & & \\
\hline
\end{tabular}

Note: variables are sorted by absolute loadings (descending). Loadings $\geq .30$ are set in bold font. $\mathbf{p o s}=$ positive scenario; $\mathbf{n e g}=$ negative scenario; $\mathbf{o t h}=$ other; sit $=$ situation .

Unconfirmed attributions are the initial ratings provided following the first statement presented; confirmed attributions are the final ratings provided when the respective attributional locus was supported by the preceding evidence; disconfirmed attributions are the final ratings provided when the respective attributional locus was not supported by the evidence. 
Table 4.17. E component loadings from CPCA no. 2 in nonclinical participants $(\mathrm{Z}=$ all observed attribution ratings, $\mathrm{G}=C A P E)$.

\begin{tabular}{lcccc}
\hline Attributions & 1 & 2 & 3 & 4 \\
\hline Self confirmed, pos & $\mathbf{0 . 8 5 6}$ & 0.075 & -0.056 & 0.187 \\
Self confirmed, neg & $\mathbf{0 . 8 4 2}$ & 0.015 & -0.141 & 0.103 \\
Oth confirmed, pos & $\mathbf{0 . 7 3 0}$ & 0.182 & 0.328 & -0.178 \\
Oth confirmed, neg & $\mathbf{0 . 5 9 3}$ & -0.015 & $\mathbf{0 . 5 1 0}$ & -0.342 \\
Sit confirmed, pos & $\mathbf{0 . 6 3 6}$ & $\mathbf{0 . 4 4 7}$ & -0.132 & -0.225 \\
Sit confirmed, neg & $\mathbf{0 . 6 0 8}$ & $\mathbf{0 . 5 5 2}$ & -0.008 & -0.174 \\
Sit unconfirmed, pos & 0.085 & $\mathbf{0 . 8 3 2}$ & 0.182 & 0.169 \\
Sit unconfirmed, neg & 0.186 & $\mathbf{0 . 8 1 9}$ & 0.098 & 0.126 \\
Sit disconfirmed, pos & 0.115 & $\mathbf{0 . 8 3 3}$ & 0.200 & 0.142 \\
Sit disconfirmed, neg & 0.017 & $\mathbf{0 . 7 7 9}$ & 0.121 & 0.182 \\
Oth unconfirmed, pos & -0.001 & 0.367 & $\mathbf{0 . 7 8 2}$ & 0.193 \\
Oth unconfirmed, neg & 0.257 & 0.071 & $\mathbf{0 . 8 3 7}$ & -0.054 \\
Oth disconfirmed, pos & -0.031 & 0.175 & $\mathbf{0 . 8 0 9}$ & 0.142 \\
Oth disconfirmed, neg & -0.381 & 0.111 & $\mathbf{0 . 6 8 5}$ & 0.209 \\
Self unconfirmed, pos & $\mathbf{0 . 5 0 8}$ & 0.042 & $\mathbf{0 . 4 6 1}$ & 0.175 \\
Self unconfirmed, neg & 0.183 & 0.333 & 0.118 & $\mathbf{0 . 6 9 1}$ \\
Self disconfirmed, pos & -0.037 & -0.072 & $\mathbf{0 . 4 7 6}$ & $\mathbf{0 . 6 5 3}$ \\
Self disconfirmed, neg & -0.161 & $\mathbf{0 . 4 1 3}$ & 0.021 & $\mathbf{0 . 7 8 3}$ \\
\hline
\end{tabular}

Note: variables are sorted to display differences/similarities with the four-component structure obtained from GSCA in Study 1. Loadings $\geq .40$ are set in bold font. Components are ordered by decreasing magnitude of total variance explained. pos = positive scenario; $\mathbf{n e g}=$ negative scenario; oth $=$ other; sit $=$ situation. Unconfirmed attributions are the initial ratings provided following the first statement presented; confirmed attributions are the final ratings provided when the respective attributional locus was supported by the preceding evidence; disconfirmed attributions are the final ratings provided when the respective attributional locus was not supported by the evidence.

\section{CPCA no. 3: Neurocognitive measures partialled out of the ASB task}

Table 4.18. below presents the distribution of variance among the elements of CPCA, after neurocognitive measures were regressed out of the ASB task. These measures accounted for $6.75 \%$ of the total variance in attribution ratings. 
Table 4.18. Distribution of variance for CPCA no. 3 in nonclinical participants $(\mathrm{Z}=$ all attribution ratings with neurocognitive measures partialled out, $\mathrm{G}=$ CAPE).

\begin{tabular}{ccccccc}
\hline & & \multicolumn{5}{c}{ Internal } \\
\cline { 3 - 7 } Portion of Variance & External & 1 & 2 & 3 & 4 & All \\
\hline Predicted $(G C-$ CAPE) & 1.43 & 1.04 & - & - & - & 1.04 \\
\% Predicted & $\mathbf{1 0 0 . 0 0}$ & $\mathbf{7 2 . 7 0}$ & - & - & - & $\mathbf{7 2 . 7 0}$ \\
\% Total & $\mathbf{7 . 9 3}$ & $\mathbf{5 . 7 6}$ & - & - & - & $\mathbf{5 . 7 6}$ \\
\hline Residual $(E)$ & 16.57 & 3.74 & 3.63 & 3.48 & 1.97 & 12.82 \\
\% Residual & $\mathbf{1 0 0 . 0 0}$ & $\mathbf{2 2 . 5 6}$ & $\mathbf{2 1 . 8 9}$ & $\mathbf{2 1 . 0 1}$ & $\mathbf{1 1 . 9 1}$ & $\mathbf{7 7 . 3 8}$ \\
\% Total & $\mathbf{9 2 . 0 7}$ & $\mathbf{2 0 . 7 7}$ & $\mathbf{2 0 . 1 5}$ & $\mathbf{1 9 . 3 5}$ & $\mathbf{1 0 . 9 7}$ & $\mathbf{7 1 . 2 4}$ \\
\hline
\end{tabular}

Note: The variances accounted for from the external analysis (regression) and by the components extracted from the internal analysis (PCA) are listed in regular font. The percentages of variance accounted for by each source are listed in bold font. $\%$ Total $=$ percentage of total variance explained by the source identified in each column. $\%$ Predicted $=$ percentage of predictable variance explained by the source identified in each column. $\%$ Residual $=$ percentage of residual variance explained by the source identified in each column. Components are ordered by decreasing magnitude of total variance explained.

Analysis of $\boldsymbol{G C}$ and $\boldsymbol{G}$. Again, one component was extracted from $G C$. This component was dominated by unconfirmed self attributions for positive scenarios (.365), unconfirmed situation attributions for negative scenarios (.320), disconfirmed situation attributions for positive scenarios (.304), and confirmed situation attributions for positive scenarios (.301), and was highly negatively correlated with depression frequency $(r=-.819)$. $G C$ component loadings and $G$ loadings are listed in Table 4.19.

Internal analysis of $\boldsymbol{E}$. PCA on the residual variance revealed a four-component solution in $E$, with the highest contributions from: (1) unsupported situation attributions, (2) confirmed attributions, (3) unsupported other attributions, and (4) unsupported self attributions (though again excluding unconfirmed self attributions for positive scenarios). $E$ component loadings are listed in Table 4.20. 
Table 4.19. GC component loadings and $\mathrm{G}$ loadings from CPCA no. 3 in nonclinical participants $(\mathrm{Z}=$ all attribution ratings with neurocognitive measures partialled out, $\mathrm{G}=$ CAPE).

\begin{tabular}{|c|c|c|c|}
\hline \multicolumn{2}{|l|}{$G C$} & \multicolumn{2}{|l|}{$G$} \\
\hline Attributions & Loadings & Symptom Dimensions & Loadings \\
\hline Self unconfirmed, pos & 0.365 & Depression & -0.819 \\
\hline Sit unconfirmed, neg & 0.320 & Positive & 0.059 \\
\hline Sit disconfirmed, pos & 0.304 & Negative & -0.006 \\
\hline Sit confirmed, pos & 0.301 & & \\
\hline Sit disconfirmed, neg & 0.263 & & \\
\hline Self unconfirmed, neg & 0.263 & & \\
\hline Self disconfirmed, pos & 0.263 & & \\
\hline Sit unconfirmed, pos & 0.259 & & \\
\hline Oth confirmed, neg & 0.250 & & \\
\hline Oth unconfirmed, neg & 0.230 & & \\
\hline Oth disconfirmed, neg & 0.219 & & \\
\hline Oth confirmed, pos & 0.218 & & \\
\hline Sit confirmed, neg & 0.209 & & \\
\hline Self confirmed, pos & 0.180 & & \\
\hline Oth unconfirmed, pos & 0.178 & & \\
\hline Self confirmed, neg & -0.101 & & \\
\hline Oth disconfirmed, pos & 0.097 & & \\
\hline Self disconfirmed, neg & 0.074 & & \\
\hline
\end{tabular}

Note: variables are sorted by absolute loadings (descending). Loadings $\geq .30$ are set in bold font $\mathbf{p o s}=$ positive scenario; $\mathbf{n e g}=$ negative scenario; oth $=$ other; sit $=$ situation . Unconfirmed attributions are the initial ratings provided following the first statement presented; confirmed attributions are the final ratings provided when the respective attributional locus was supported by the preceding evidence; disconfirmed attributions are the final ratings provided when the respective attributional locus was not supported by the evidence. 
Table 4.20. E component loadings from CPCA no. 3 in nonclinical participants $(\mathrm{Z}=$ all attribution ratings with neurocognitive measures partialled out, $\mathrm{G}=C A P E)$.

\begin{tabular}{lcccc}
\hline Attributions & 1 & 2 & 3 & 4 \\
\hline Sit unconfirmed, pos & $\mathbf{0 . 8 3 7}$ & 0.108 & 0.184 & 0.147 \\
Sit unconfirmed, neg & $\mathbf{0 . 8 1 7}$ & 0.231 & 0.087 & 0.123 \\
Sit disconfirmed, pos & $\mathbf{0 . 8 3 9}$ & 0.118 & 0.203 & 0.122 \\
Sit disconfirmed, neg & $\mathbf{0 . 7 7 7}$ & 0.022 & 0.115 & 0.206 \\
Self confirmed, pos & 0.120 & $\mathbf{0 . 8 5 4}$ & -0.061 & 0.198 \\
Self confirmed, neg & 0.073 & $\mathbf{0 . 8 3 3}$ & -0.154 & 0.121 \\
Oth confirmed, pos & 0.234 & $\mathbf{0 . 7 1 1}$ & 0.337 & -0.168 \\
Oth confirmed, neg & 0.039 & $\mathbf{0 . 5 7 1}$ & $\mathbf{0 . 5 2 9}$ & -0.342 \\
Sit confirmed, pos & $\mathbf{0 . 4 8 8}$ & $\mathbf{0 . 6 0 0}$ & -0.129 & -0.239 \\
Sit confirmed, neg & $\mathbf{0 . 5 9 0}$ & $\mathbf{0 . 5 8 3}$ & -0.006 & -0.146 \\
Oth unconfirmed, pos & 0.337 & 0.026 & $\mathbf{0 . 8 0 3}$ & 0.138 \\
Oth unconfirmed, neg & 0.060 & 0.282 & $\mathbf{0 . 8 3 5}$ & -0.063 \\
Oth disconfirmed, pos & 0.174 & -0.049 & $\mathbf{0 . 8 1 8}$ & 0.156 \\
Oth disconfirmed, neg & 0.067 & -0.356 & $\mathbf{0 . 6 9 4}$ & 0.198 \\
Self unconfirmed, pos & 0.034 & $\mathbf{0 . 5 4 4}$ & $\mathbf{0 . 4 5 7}$ & 0.169 \\
Self unconfirmed, neg & 0.298 & 0.260 & 0.117 & $\mathbf{0 . 6 5 6}$ \\
Self disconfirmed, pos & -0.108 & 0.002 & $\mathbf{0 . 4 8 2}$ & $\mathbf{0 . 6 5 3}$ \\
Self disconfirmed, neg & 0.384 & -0.146 & -0.003 & $\mathbf{0 . 7 9 5}$ \\
\hline
\end{tabular}

Note: variables are sorted to display differences/similarities with the four-component structure obtained from GSCA in Study 1. Loadings $\geq .40$ are set in bold font. Components are ordered by decreasing magnitude of total variance explained. pos = positive scenario; $\mathbf{n e g}=$ negative scenario; oth $=$ other; $\mathbf{s i t}=$ situation. Unconfirmed attributions are the initial ratings provided following the first statement presented; confirmed attributions are the final ratings provided when the respective attributional locus was supported by the preceding evidence; disconfirmed attributions are the final ratings provided when the respective attributional locus was not supported by the evidence.

To summarize the results observed in the nonclinical sample, CPCAs revealed a general pattern of overlap among unconfirmed self attributions for positive scenarios and (unsupported) situation attributions in the CAPE-predicted solutions, which was negatively correlated with depression. No other symptom dimensions on the CAPE showed high contributions to the predicted solution.

\subsection{Summary of Study 2}

Study 2 aimed to identify components of the ASB task that emerge from specific psychopathology and schizotypical traits. Regardless of whether $Z$ consisted of initial attribution 
ratings only, all observed attribution ratings, or all attribution ratings with variance from neurocognitive measures removed, the SSPI-predicted solution displayed overlap among unsupported self attributions for negative scenarios and situation attributions for positive and negative scenarios, which correlated with disorganized symptoms and elated mood. A second component in CPCA with all observed attribution ratings was dominated by other-person attributions, and correlated with depression and anxiety; however, this component did not remain after regressing neurocognitive measures out of the ASB task. Therefore, a BADE-like component emerged from the predicted solutions as expected (reflected in overlap among unconfirmed and disconfirmed attributions), but it did not appear to reflect self-serving or personalizing biases, and it was not highly correlated with delusions. Although paranoid delusions showed a minor contribution to this component, this relationship did not remain after neurocognitive function was partialled out of the ASB task.

The CAPE-predicted solutions displayed an interesting consolidation with the results obtained with the SSPI; that is, the highest contributions to the component extracted from the predicted solutions were unconfirmed (but not disconfirmed) self attributions for positive scenarios and unsupported situation attributions for positive and negative scenarios, and was highly negatively correlated with frequency of depression. The negative correlation with situation attributions is consistent with the notion that mania is, in a sense, the "opposite" of depression. 


\section{Chapter 5: Discussion}

\subsection{Summary}

Extreme attributional biases have been implicated in mental illness, particularly in paranoia and depression. A "defense" model of paranoid delusions has been proposed by Bentall et al. (1994), who suggest that paranoia arises from an extreme self-serving bias with a characteristic tendency to blame other people rather than the circumstances for negative events; this initially serves to defend against threats to self-esteem, but ultimately gives rise to the perception of negative intentions in other people. By contrast, depressed individuals are more likely to assign blame to themselves and credit to external causes (Kinderman \& Bentall, 1997).

Most studies on attributional biases in psychosis have focused exclusively on paranoid delusions, failing to take into account the heterogeneity of psychotic illness. Further, current measures of attributional style do not assess an individual's ability to use new information to revise their initial assumptions; that is, existing measures of attributional bias only engage one aspect of this cognitive bias (belief formation) and fail to consider another crucial aspect (belief maintenance). Given the extensive literature on associations between a general BADE and delusions (Riccaboni et al., 2012; Sanford et al., 2014; Speechley et al., 2012; Woodward et al., 2007; Woodward, Moritz, \& Chen, 2006; Woodward, Moritz, Cuttler, et al., 2006), the consideration of potential additive or multiplicative effects with respect to the BADE, attributional biases, and associations with (paranoid) delusions may be beneficial for understanding the cognitive underpinnings of delusions.

The present research attempted to address these shortcomings with the use of the ASB

task, a novel task that combines a standard attributional bias paradigm (IPSAQ) with a test of hypothesis judgement and reasoning (BADE). The specific objectives of this research included 
(1) establishing the component structure of the ASB task, (2) examining attributional biases that underlie differences between healthy individuals, schizophrenia patients, bipolar disorder patients, and symptom-based patient subgroups (based on severity of paranoid delusions or depression), and (3) examining attributional biases that are predictable from symptom measures. These goals were achieved primarily using GSCA (objective no. 1) and CPCA (objectives no. 2 and 3).

In Study 1, which examined the component structure of the ASB task and the extent to which group differences could explain responding, it was predicted that severely paranoid schizophrenia patients would display an enhanced externalizing and personalizing bias (in line with the defense model of paranoia), while severely depressed schizophrenia and bipolar disorder patients were expected to display an attenuated self-serving bias and/or increased selfblame. A four-component task structure was obtained, as suggested first by exploratory PCA, and then tested with multi-group GSCA. One component included all confirmed attributions (i.e., ratings for attributions that were supported by the evidence provided, together labelled "supported" attributions), and the three other components each included unconfirmed and disconfirmed (together labelled "unsupported") attributions for each causal locus. However, no differences between diagnostic- or symptom-based participant groups were found.

Studies 2 and 3 investigated components of the ASB task that are directly predictable from symptoms of psychosis (measured with the SSPI) or schizotypical traits (measured with the CAPE). The component structures predicted by clinical symptoms in patients and schizotypical traits in nonclinical participants accounted for $11-18 \%$ and $6-8 \%$ of the variance in responding on the ASB task, respectively; however, paranoid delusions did not predict self-serving or personalizing biases. Rather, symptoms of mania (overactivity, elated mood) and disorganization 
(disordered thought, inappropriate affect) in the patient sample predicted a component dominated by unsupported situation attributions and self-blame, while frequency of depression in the nonclinical sample was negatively correlated with a component dominated by unsupported situation ratings and self-credit.

\subsection{Cognitive Operations of the ASB Task}

Analysis of the underlying component structure of the ASB task demonstrated overlap among unconfirmed and disconfirmed attributions, which was distinct for each attributional locus, but did not differentiate between positive and negative scenarios. Conversely, all "confirmed" attributions correlated with each other, regardless of attributional locus or whether the scenario was positive or negative. Therefore, although a self-serving and personalizing bias was reflected in mean initial attribution ratings overall, individual differences in responding were better captured by the variance in ratings for the unsupported attributions for a given attributional locus, and on all supported (i.e., confirmed) attributions as a whole. The shared variance reflected in each "unsupported" component cannot be fully explained by positive correlations between participants' initial and final ratings, as this overlap was specific to disconfirmed attributions; ratings for confirmed attributions appeared to be independent of attributional locus or respective initial ratings, suggesting that the "unsupported" component patterns emerge from some form of attributional bias or willingness to integrate (socially-relevant) information.

Components obtained from unconstrained PCA are not optimized to be predictable from the independent variables of interest (i.e., symptoms of psychosis and schizotypical traits), and as such, the component solutions obtained from the SSPI- and CAPE-predicted data were strikingly different from that obtained from the observed data. This does not necessarily imply that the independent variable of interest is a poor predictor of task performance (although this may be the 
case as well, such as in Study 1), but rather that the primary underlying dimensions of the dependent variable are not predictable from the independent variables. This is important to note, given that such components typically form the subscales of standard behavioural assessments; that is, one might not find a significant correlation between an independent variable of interest and the standard dimensions of a given assessment, even though this variable may have a meaningful relationship with some other aspect of task performance. Moreover, the standard dimensions may be predictable from independent variables not studied in the analysis in question.

It is important to note that, unlike the traditional BADE paradigm, the ASB task does not make use of explicit "lure" interpretations (i.e., interpretations of the given scenario that seem highly plausible initially, and then are "disproved" by the subsequently presented evidence). It was assumed that participants would implicitly consider the "self" attribution as being the most plausible for positive scenarios, and external attributions the most plausible for negative scenarios. In this sense, self attributions for positive scenarios and external attributions for negative scenarios were conceptualized as "lures" that are disproved on trials where an alternate attribution is implied in the second statement presented (e.g., "You aced a major exam... Now imagine that everyone in the class aced the exam"). Indeed, such a self-serving bias was evident in participants' mean initial attribution ratings. However, the effect of disconfirming evidence may have been more robust if the scenarios had been explicitly divided into trials where the cause was ambiguous at the start (as in the current ASB task), trials where a "lure" attribution was subsequently disconfirmed, and trials where a "lure" attribution was subsequently confirmed; such a setup would be more comparable to the design of the traditional BADE task. 
The components obtained in the ASB task and the BADE task may reflect similar, but not identical, cognitive operations. Although it can be argued that the "unsupported" components of the ASB task are similar to a BADE in that they specifically include disconfirmed attributions, these components did not include negatively-loaded contributions from confirmed attributions, which would be more consistent with the evidence integration component on the traditional BADE task. Such a pattern in the BADE task is thought to reflect both an unwillingness to down-rate initially plausible but ultimately disconfirmed interpretations and unwillingness to uprate initially less plausible interpretations that are revealed to be true; therefore, the unsupported components in the ASB task may capture the unwillingness to down-rate disconfirmed attributions, but no reflection of unwillingness to up-rate confirmed attributions. In addition, although the "supported" component appears on the surface to resemble the BADE "conservatism" component (in the sense that these components are dominated by items where high ratings would be expected), the "supported" component, unlike conservatism, did not appear to be specifically driven by low ratings. Again, this may be a result of not having explicit "lures" built into the task, as the conservatism component observed in the traditional BADE task does not only reflect conservative ratings on the "true" interpretation, but also includes high contributions from the initial "lure" ratings. The strength of the initial evidence presented to the participants may be key to understanding the cognitive processes engaged and reflected in the component structures of the BADE tasks.

One noteworthy difference between the ASB and previously employed attributional style tasks such as the IPSAQ is that participants were not asked to provide a possible explanation for the event presented to them. This part of the paradigm was excluded because previous studies have demonstrated that independent raters' evaluations of participants' causal statements 
frequently do not match the attributions chosen by the participants themselves (Martin \& Penn, 2002; Randall et al., 2003), and so it was not expected that this would provide an advantage over simply asking the participant to provide ratings based on intuition. However, asking participants to provide a concrete hypothesis could potentially have an added impact on their subsequent attribution ratings; that is, participants' own explanations would serve as the "lure" in cases where their initial highest-rated attribution is not supported by the follow-up statement. Similar to the above speculation regarding the use of more explicit "lure" interpretations utilized in the ASB task, perhaps the process of imagining and providing a concrete hypothesis would make individuals more inclined to persist in their attributional judgements. This could be an interesting effect to examine in future studies.

Other domains of social cognition are likely to be engaged in the ASB task. For example, social perception (i.e., ability to identify social roles, societal rules, and social context) may be important for understanding the content of the task and using this information to make causal inferences. In order to fully understand what is being measured on the ASB task and to further elucidate the cognitive processes being engaged, future research should investigate overlap between responding on the ASB task and other cognitive tasks. Factor analyses including a variety of measures (including, for example, social cognitive measures of theory of mind, social knowledge and perception, and cognitive bias measures such as jumping to conclusions and general BADE) may help to elucidate what is uniquely involved in forming causal attributions on the ASB task, and what overlaps with other cognitive measures.

\subsection{Group Comparisons}

The present findings did not replicate previous research demonstrating either a selfserving/personalizing bias in paranoid patients or attenuation of a self-serving bias in depressed 
patients, and the hypothesis-based groups accounted for a negligible amount of variance in performance on the ASB task. It is possible that group differences would have been observed if, for example, symptom-based subgroups had been formed based on severity of disordered thought rather than paranoid delusions or depression, as neither paranoia nor depression had a high contribution to the SSPI-predicted solutions in this sample of patients. However, this would still have left out a considerable amount of information with respect to the relative contributions of other symptoms measured with the SSPI. These results highlight the importance of considering the heterogeneity of psychotic illness rather than relying on broad diagnostic groups, at least in studies of symptom-based cognitive processes.

\subsection{Symptom-Related Attributions}

\subsubsection{Patients}

Symptoms of mania and disorganization appeared to be the strongest predictors of attributional biases in schizophrenia and bipolar disorder patients. This was unexpected, given previous research demonstrating a specific association between delusions and attributional biases and a BADE. It has been suggested that an enhanced BADE in delusional patients relies on the salience of evidence-hypothesis matches (i.e., the strength of the evidence presented; Balzan, Delfabbro, Galletly, \& Woodward, 2012; Balzan, Delfabbro, Galletly, \& Woodward, 2013); there is no obviously salient evidence-hypothesis match when participants make their initial ratings on the ASB task, as the cause is intended to be ambiguous at the start of the trial. It is possible that the initial presentation of the scenario must imply a specific causal attribution in order for delusional patients (and other individuals) to be led into a firm belief (either with the use of lures built into the task, or by asking participants to provide a specific hypothesis, as noted above). 
Regarding the involvement of external-situational attributions in the predicted solutions, it can be speculated that such a tendency could be related to impaired theory of mind or related domain of social cognition; that is, if an individual has difficulty imagining him or herself in a scenario that s/he may never have experienced, while also having difficulty considering another person's involvement in the event, s/he may regard "chance" as the most likely cause. The apparent unwillingness to adjust one's ratings following disconfirming evidence could then reflect an inability to make a connection between the socially-relevant information provided and the attribution it is meant to imply, due to a specific impairment in social cognition. Indeed, an impairment in the ability to use contextual information to make causal inferences in social cognition tasks has been demonstrated in schizophrenia and bipolar disorder (Baez et al., 2013). With respect to the disorganized symptoms implicated in the present findings, this is consistent with Hardy-Baylé and colleague's model of the cognitive mechanisms of schizophrenic disorganization (Hardy-Baylé, Sarfati, \& Passerieux, 2003), which proposes that two primary cognitive deficits underlie symptoms of disorganization: (1) an inability to integrate contextual information, and (2) a deficit in theory of mind. Such a deficit could accompany mania as well. A subtle (but important) difference between using contextual information to make a causalattributional inference and integrating evidence to revise a specific belief may explain the lack of involvement of reality distortion symptoms in the present research (though paranoid delusions contributed to some extent in Study 2, paranoia may rely more heavily on social cognition than other delusional themes measured on the SSPI).

The overlap between self-blame and situational attributions in the predicted solutions is seemingly contradictory, but not altogether surprising in the context of impaired social cognition. Self-blame is a generally dysfunctional form of cognition which, although normally associated 
with depression, may be associated with high general psychopathology (Mizrahi, Addington, Remington, \& Kapur, 2008). In this sense, the SSPI-predicted solution including high contributions from self-blame is consistent with the notion of such an attributional bias being broadly exhibited in mental illness.

Recall that, initially, a second component was extracted from the CPCA with all attribution ratings included, but was not retained after the variance from neurocognitive measures was removed from the ASB task. This component was dominated by other-person attributions, and was most highly correlated with depression. It is possible that this component was driven by patients with relatively high neurocognitive functioning, and so the instability of this component may be explained by the removal of shared variance among neurocognitive functioning, other-person attributions, and depressed mood.

\subsubsection{Nonclinical participants}

The attributional biases predicted by schizotypical traits in nonclinical participants present an interesting consolidation with those observed in patients. Although the negative correlation with situation attributions is somewhat difficult to explain, it is interesting that depression in these individuals displayed an opposing effect from that of elated mood and overactivity in patients. To some degree, depression can be conceptualized as the "opposite" of mania, and so these predicted component patterns may be related and potentially capture some common relationship with a mood-related dimension. The negative correlation between frequency of depression and self-credit is consistent with previous research demonstrating such an attenuation of a self-serving bias in depressed individuals. Evidently, self and situation attributions are important in some way in the connection between the ASB task and psychopathology (and perhaps affective symptoms in particular, in both clinical and healthy 
samples), but future research is required to better understand what explains or underlies this relationship.

\subsection{Limitations}

Symptoms in schizophrenia and bipolar disorder cycle through active states and remissions over time, and therefore the information gained from studying symptom-related measures at a single time point is limited. As in any cross-sectional analysis, the present research could not examine whether the observed correlations would remain over time as symptoms changed. Longitudinal studies investigating correlations between changes in symptom severity and (potential) changes in attributions could more rigorously investigate whether the observed relationships between attributional biases and symptoms are state-dependent cognitive processes that change with fluctuations in symptom severity (as observed in jumping to conclusions and delusions; Sanford et al., 2013; Woodward et al., 2009), or are traits characteristic of patients prone to severe pathology.

The present research may not fully explain the mechanisms underlying the responses observed on the ASB task. Another (social) cognitive domain, such as social perception, may have an impact on participants' ability to make use of the task content to form conclusions about an event. However, no other tests of social cognition were administered to examine this possibility. Therefore, further analyses of the ASB task are required before firm conclusions can be made about the abilities captured in the measures derived from this task. As suggested above, factor analyses including a wide range of social- and neuro-cognitive measures will be valuable for such investigation.

It should be noted that a discrepancy between "poor me" and "bad me" paranoia has been described. While the former is characterized by paranoid themes in which the perceived 
persecution is felt to be undeserved, the latter is characterized by a perception of deserved persecution. It has been suggested that only the "poor me" type arises from a defensive attributional style, while the "bad me" type is more closely related to depression and thus is accompanied by a depressive, non-self-serving bias (Melo, Taylor, \& Bentall, 2006). Unfortunately, the SSPI measure of paranoia does not make such a distinction, and the present sample size was not large enough to differentiate between non-depressed paranoid, depressedparanoid, and non-paranoid depressed patients. It is possible that the SSPI measure of paranoia was simply too broad to demonstrate a relationship between paranoid delusions and a selfserving/personalizing bias.

Results from PCA and regression may be sensitive to the specific characteristics of the sample of interest, and so replications in larger and varied samples are necessary to provide a better understanding of the generalizability of the reported analyses. Minimum sample size recommendations for good recovery of population factors in PCA and factor analysis vary widely (MacCallum, Widaman, Zhang, \& Hong, 1999). Further complicating this is the possibility that typical recommendations for unconstrained PCA may not apply to constrained PCA. The unconstrained PCA on all attribution ratings in Study 1 was perhaps the most important with respect to recovery of population factors, as this formed the basis of the latent structure tested with GSCA; in this analysis, the $n$ (participants) to $p$ (variables) ratio was 8.33 (150:18), which is above the minimum recommended by some authors (Cattell, 1978; Gorsuch, 1983) but not others (Everitt, 1975). Regarding the use of small sample sizes in multiple regression (performed in the external analysis of CPCA), there is a known risk of an inflated $R^{2}$ value with a greater number of independent variables. A potentially serious case of this in the reported analyses was in the patient sample in Study 2, where $G$ (the SSPI) consisted of 21 
variables and accounted for approximately $25 \%$ of the variance in $Z$. However, the variance of interest was from the subsequent reduction of the predicted data into its component structure and its correlations with each of the variables in $G$, which revealed the few symptoms providing the greatest contribution to the predicted solution. Therefore, CPCA overcomes this limitation by bringing out only the reliable portion of predictable variance, which is likely to be considerably smaller than the total amount of predictable variance when the number of independent variables is close to the number of participants. Nevertheless, it is important to keep in mind that the overall percentage of predictable variance included contributions from symptoms that were not highly correlated with the predicted component solution, and, at this stage, the present findings should be interpreted specifically in the context of this particular sample of participants.

\subsection{Future Directions}

There has been an increased interest in the development of social-cognitive therapies for patients with psychotic illness, and research in this area has been promising (Fiszdon \& Reddy, 2012; Horan et al., 2008; Kurtz \& Richardson, 2012; Marsh et al., 2013). For example, a metacognitive training module for attributional style has been developed, which involves encouraging patients to provide several possible causes for a given event rather than assigning responsibility to one single source (Moritz et al., 2005). Future clinical research could incorporate the ASB task to determine whether it can predict which patients are most likely to benefit from such treatment, and if attributional biases correlate with resolution of symptoms following treatment. As previously noted, for example, an extreme attributional style in patients with bipolar disorder may predict relapse of manic/hypomanic episodes and longer time to recover during depressive episodes (Stange, Sylvia, da Silva Magalhães, Frank, et al., 2013; Stange, Sylvia, da Silva Magalhães, Miklowitz, et al., 2013). In addition, it has been 
demonstrated that schizophrenia patients with a low self-serving bias may be less responsive to antipsychotic treatment (Mizrahi et al., 2008). Therefore, it is crucial that we further our understanding of this area of cognition in the clinical setting so that such attributional biasfocused treatments and assessments can be refined and implemented. Moreover, as noted in the discussion of limitations, such longitudinal clinical studies would provide the opportunity to investigate whether attributional biases are state-dependent correlates of psychotic symptoms, or are merely characteristic of patients prone to severe psychopathology.

Incorporating neuroimaging methods to explore relationships between networks of brain activity and task performance is crucial for understanding which networks correlate with the cognitive measure of interest (i.e., attributional reasoning), and how these may be altered in psychosis. If previously identified networks are recruited in a cognitive task such as the ASB, this could help to further our understanding of the cognitive processes involved, and vice verse. Though little neuroimaging research has been carried out in attributional style, some notable experiments have been reported. For example, Blackwood et al. (2003) implemented an IPSAQ task adapted for functional magnetic resonance imaging (fMRI) administration and found that determination of self-responsibility recruited brain areas previously associated with action simulation (e.g., bilateral premotor cortex and cerebellum), suggesting that the involvement of simple internal models of goal-directed action are implicated in this seemingly higher-order cognitive process. In another fMRI study investigating attributional reasoning in healthy individuals and patients with major depression, Seidel et al. (2012) found that, behaviourally, healthy individuals displayed a self-serving bias, while depressed patients displayed more balanced attributions, as expected. Interestingly, activity in a fronto-temporal network (involving the left temporal pole, and left dorsomedial and right ventrolateral prefrontal cortex) was 
associated with attributional response conflict; that is, this activity occurred when healthy participants made non-self-serving responses and when patients with depression made selfserving responses. It can be speculated that such response conflict-related activity could be recruited in the ASB task when participants must revise their initial attribution ratings to be more consistent with an unfavourable causal attribution such as self-blame.

\subsection{Conclusions}

The fixedness of beliefs may be observed in the context of attributional reasoning, but may amount to a general bias towards a given attributional locus as measured with the ASB task. This highlights the importance of being aware of the measures employed in a given cognitive task, as individual attribution ratings would not capture the overlap among the different (but evidently related) attribution scores displayed in each component. Additional research is necessary to elucidate the cognitive processes engaged in the ASB task, as other domains of social cognition (e.g., theory of mind, social perception) or some other cognitive bias (e.g., jumping to conclusions) are likely to be implicated in task performance as well.

The group differences employed in Study 1 did not account for a meaningful amount of variance in responses on the ASB task. Moreover, the results from Study 2 suggest that other such groupings based on individual symptoms would also have left out meaningful information regarding the relative contributions of all SSPI symptoms. Therefore, a multivariate symptombased approach may be more appropriate for future research in attributional biases and psychosis.

Though previously unobserved in attributional bias research, symptoms of mania and disorganization appear to have a high involvement in the connection between psychopathology and attributional reasoning, at least in schizophrenia and bipolar disorder patients. Such 
symptoms may be specifically implicated in a cognitive process involving a tendency for exhibiting self-blame and attributing the cause of events to chance when contextual information suggests otherwise. Such an impairment may not necessarily reflect a bias against beliefdisconfirming information (as is observed in delusions), but perhaps an inability to make a connection between contextual information provided and the causation implied due to a specific deficit in social cognition. Although neither manic symptoms nor disorganization were measurable in healthy participants, a similar emergence of situational and self attributions was observed in the CAPE-predicted solution. However, this was negatively correlated with frequency of depression, and included the contribution of self-credit rather than self-blame. The patterns of correlations between symptoms and situation attributions in patients and nonclinical participants are consistent with the notion that mania is, in a sense, the "opposite" of depression. Therefore, self and situation attributions on the ASB task appear to be particularly important in the relationship between attributional reasoning and symptoms of psychosis/schizotypical traits; as noted above, however, further research is required to determine the specific cognitive mechanisms underlying these patterns.

The present research has shed light on largely overlooked dimensions of psychosis in attributional bias research (i.e., mania and disorganization) and demonstrated distinctive patterns of symptom-based attributional biases. Evidently, attributional bias warrants further study in the context of psychosis, particularly using an approach that considers a wide range of symptoms. Future research with the ASB task and other cognitive measures will further our understanding of context-dependent causal inferences and the fixedness of attributional biases. Overall, these findings point to a promising avenue of research in social cognition and cognitive biases in psychosis. 


\section{References}

Abramson, L. Y., Seligman, M. E. P., \& Teasdale, J. D. (1978). Learned helplessness in humans: Critique and reformulation. Journal of Abnormal Psychology, 87, 49-74.

American Psychiatric Association. (2000). Diagnostic and Statistical Manual (Text Revised, 4th ed.). Washington, DC: American Psychiatric Association.

Baez, S., Herrera, E., Villarin, L., Theil, D., Gonzalez-Gadea, M. L., Gomez, P., . . Ibañez, A. M. (2013). Contextual social cognition impairments in schizophrenia and bipolar disorder. PLoS One, 8, e57664.

Balzan, R. P., Delfabbro, P. H., Galletly, C., \& Woodward, T. S. (2012). Reasoning heuristics across the psychosis continuum: The contribution of hypersalient evidence-hypothesis matches. Cognitive Neuropsychiatry, 17, 431-450.

Balzan, R. P., Delfabbro, P. H., Galletly, C. A., \& Woodward, T. S. (2013). Confirmation biases across the psychosis continuum: The contribution of hypersalient evidence-hypothesis matches. British Journal of Clinical Psychology, 52, 53-69.

Bandalos, D., \& Boehm-Kaufman, M. (2008). Four common misconceptions in exploratory factor analysis. In C. E. Lance \& R. J. Vandenberg (Eds.), Statistical and Methodological Myths and Urban Legends: Doctrine, Verity and Fable in the Organizational and Social Sciences. New York, NY: Taylor \& Francis Group.

Bell, V., Halligan, P. W., \& Ellis, H. D. (2006). Explaining delusions: A cognitive perspective. Trends in Cognitive Sciences, 10, 219-226.

Bentall, R. P., Kinderman, P., \& Kaney, S. (1994). The self, attributional processes and abnormal beliefs: Towards a model of persecutory delusions. Behavior Research and Therapy, 32, $331-341$. 
Blackwood, N. J., Bentall, R. P., ffytche, D. H., Simmons, A., Murray, R. M., \& Howard, R. J. (2003). Self-responsibility and the self-serving bias: An fMRI investigation of causal attributions. NeuroImage, 20, 1076-1085.

Blackwood, N. J., Howard, R. J., Bentall, R. P., \& Murray, R. M. (2001). Cognitive neuropsychiatric models of persecutory delusions. The American Journal of Psychiatry, $158,527-539$.

Blom, J. D., \& Van Praag, H. M. (2011). Schizophrenia: It's broken and it can't be fixed. A conceptual analysis at the centenary of Bleuler's Dementia praecox oder Gruppe der Schizophrenien. The Israel Journal of Psychiatry and Related Sciences, 48, 240-251.

Brunet-Gouet, E., \& Decety, J. (2006). Social brain dysfunctions in schizophrenia: A review of neuroimaging studies. Psychiatry Research: Neuroimaging, 148, 75-92.

Buchy, L., Woodward, T. S., \& Liotti, M. (2007). A cognitive bias against disconfirmatory evidence (BADE) is associated with schizotypal traits. Schizophrenia Research, 90, 334337.

Burns, J. K. (2006). Psychosis: A costly by-product of social brain evolution in Homo sapiens. Progress in Neuro-Psychopharmacology and Biological Psychiatry, 30, 797-814.

Byerly, M. J., Nakonezny, P. A., \& Lescouflair, E. (2007). Antipsychotic medication adherence in schizophrenia. Psychiatric Clinics of North America, 30, 437-452.

Byrne, A., \& MacLeod, A. K. (1997). Attributions and accessibility of explanations for future events in anxiety and depression. British Journal of Clinical Psychology, 36, 505-520.

Campbell, W. K., \& Sedikides, C. (1999). Self-threat magnifies the self-serving bias: A metaanalytic integration. Review of General Psychology, 3, 23-43. 
Cattell, R. B. (1966). The scree test for the number of factors. Multivariate Behavioral Research, $1,245-276$.

Cattell, R. B. (1978). The scientific use of factor analysis in behavioral and life sciences. New York: Plenum Press.

Cattell, R. B., \& Vogelmann, S. (1977). A comprehensive trial of the scree and kg criteria for determining the number of factors. Multivariate Behavioral Research, 12, 289-325.

Cliff, N. (1988). The eigenvalues-greater-than-one rule and the reliability of components. Psychological Bulletin, 103, 276-279.

Colbert, S., Peters, E. R., \& Garety, P. A. (2010). Delusions and belief flexibility in psychosis. Psychology and Psychotherapy: Theory, Research and Practice, 83, 45-57.

Combs, D. R., \& Penn, D. L. (2008). Social cognition in paranoia. In D. Freeman, R. Bentall, \& P. Garety (Eds.), Persecutory Delusions: Assessment, Theory, and Treatment (pp. 175205): Oxford University Press.

Combs, D. R., Penn, D. L., Michael, C. O., Basso, M. R., Wiedeman, R., Siebenmorgan, M., .. . Chapman, D. (2009). Perceptions of hostility by persons with and without persecutory delusions. Cognitive Neuropsychiatry, 14, 30-52.

Combs, D. R., Penn, D. L., Wicher, M., \& Waldheter, E. (2007). The Ambiguous Intentions Hostility Questionnaire (AIHQ): A new measure for evaluating hostile social-cognitive biases in paranoia. Cognitive Neuropsychiatry, 12, 128-143.

Couture, S. M., Penn, D. L., \& Roberts, D. L. (2006). The functional significance of social cognition in schizophrenia: A review. Schizophrenia Bulletin, 32, S44-S63. 
Das, P., Lagopoulos, J., Coulston, C. M., Henderson, A. F., \& Malhi, G. S. (2012). Mentalizing impairment in schizophrenia: A functional MRI study. Schizophrenia Research, 134, $158-164$.

Diez-Alegría, C., Vázquez, C., Nieto-Moreno, M., Valiente, C., \& Fuentenebro, F. (2006). Personalizing and externalizing biases in deluded and depressed patients: Are attributional biases a stable and specific characteristic of delusions? British Journal of Clinical Psychology, 45, 531-544.

Donohoe, G., Duignan, A., Hargreaves, A., Morris, D. W., Rose, E., Robertson, D., .. Corvin, A. (2012). Social cognition in bipolar disorder versus schizophrenia: Comparability in mental state decoding deficits. Bipolar Disorders, 14, 743-748.

Efron, B. (1981). Nonparametric estimates of standard error: The jackknife, the bootstrap and other methods. Biometrika, 68, 589-599.

Everitt, B. S. (1975). Multivariate analysis: The need for data, and other problems. British Journal of Psychiatry, 126, 237-240.

Fear, C., Sharp, H., \& Healhy, D. (1996). Cognitive processes in delusional disorders. British Journal of Psychiatry, 168, 61-67.

Fett, A.-K. J., Viechtbauer, W., Dominguez, M.-d.-G., Penn, D. L., van Os, J., \& Krabbendam, L. (2011). The relationship between neurocognition and social cognition with functional outcomes in schizophrenia: A meta-analysis. Neuroscience and Biobehavioral Reviews, $35,573-588$.

Fiszdon, J. M., \& Reddy, L. F. (2012). Review of social cognitive treatments for psychosis. Clinical Psychology Review, 32, 724-740. 
Garety, P. A., \& Freeman, D. (1999). Cognitive approaches to delusions: A critical review of theories and evidence. British Journal of Clinical Psychology, 38, 113-154.

Gorsuch, R. L. (1983). Factor analysis (2 ed.). Hillsdale, New Jersey: Lawrence Erlbaum Associates.

Grant, D. A., \& Berg, E. A. (1948). A behavioral analysis of degree of reinforcement and ease of shifting to new responses in a Weigl-type card-sorting problem. Journal of Experimental Psychology, 38, 404-411.

Green, M. F., Penn, D. L., Bentall, R., Carpenter, W. T., Gaebel, W., Gur, R. C., . . Heinssen, R. (2008). Social cognition in schizophrenia: An NIMH workshop on definitions, assessment, and research opportunities. Schizophrenia Bulletin, 34, 1211-1220.

Hardy-Baylé, M.-C., Sarfati, Y., \& Passerieux, C. (2003). The cognitive basis of disorganization symptomatology in schizophrenia and its clinical correlates: Toward a pathogenetic approach to disorganization. Schizophrenia Bulletin, 29, 459-471.

Higgins, E. T. (1987). Self-discrepancy: A theory relating self and affect. Psychological Review, 94, 319-340.

Horan, W. P., Kern, R. S., Green, M. F., \& Penn, D. L. (2008). Social cognition training for individuals with schizophrenia: Emerging evidence. American Journal of Psychiatric Rehabilitation, 11, 205-252.

Hunter, M. A., \& Takane, Y. (2002). Constrained principal component analysis: Various applications. Journal of Educational and Behavioral Statistics, 27, 105-145.

Hwang, H., \& Takane, Y. (2004). Generalized Structured Component Analysis. Psychometrika, $69,81-99$. 
Jackson, D. A. (1993). Stopping rules in principal components analysis: A comparison of heuristical and statistical approaches. Ecology, 74, 2204-2214.

Kaney, S., \& Bentall, R. P. (1989). Persecutory delusions and attributional style. British Journal of Medical Psychology, 62, 191-198.

Kaufman, A. S. (1990). Kaufman Brief Intelligence Test: KBIT: AGS, American Guidance Service.

Kinderman, P., \& Bentall, R. P. (1996a). A new measure of causal locus: The Internal, Personal and Situational Attributions Questionnaire. Personality and Individual Differences, 20, 261-264.

Kinderman, P., \& Bentall, R. P. (1996b). Self-discrepancies and persecutory delusions: Evidence for a model of paranoid ideation. Journal of Abnormal Psychology, 105, 106-113.

Kinderman, P., \& Bentall, R. P. (1997). Causal attributions in paranoia and depression: Internal, personal, and situational attributions for negative events. Journal of Abnormal Psychology, 106, 341-345.

Konings, M., Bak, M., Hanssen, M., van Os, J., \& Krabbendam, L. (2006). Validity and reliability of the CAPE: A self-report instrument for the measurement of psychotic experiences in the general population. Acta Psychiatrica Scandinavica, 114, 55-61.

Kurtz, M. M., \& Richardson, C. L. (2012). Social cognitive training for schizophrenia: A metaanalytic investigation of controlled research. Schizophrenia Bulletin, 38, 1092-1104.

Langdon, R., Ward, P. B., \& Coltheart, M. (2010). Reasoning anomalies associated with delusions in schizophrenia. Schizophrenia Bulletin, 36, 321-330. 
Leucht, S., Arbter, D., Engel, R. R., Kissling, W., \& Davis, J. M. (2009). How effective are second-generation antipsychotic drugs? A meta-analysis of placebo-controlled trials. Molecular Psychiatry, 14, 429-447.

Liddle, P. F., Ngan, E. T. C., Duffield, G., \& Warren, A. J. (2002). Signs and Symptoms of Psychotic Illness (SSPI): A rating scale. The British Journal of Psychiatry, 180, 45-50.

Lincoln, T. M., Mehl, S., Kesting, M.-L., \& Rief, W. (2011). Negative symptoms and social cognition: Identifying targets for psychological interventions. Schizophrenia Bulletin, 37, S23-S32.

Loong, J. (1991). Wisconsin Card Sorting Test - IBM Version. San Luis Obispo, CA: Wang Neuropsychological Laboratories.

Lyon, H. M., Kaney, S., \& Bentall, R. P. (1994). The defensive function of persecutory delusions: Evidence from attribution tasks. British Journal of Psychiatry, 164, 637-646.

MacCallum, R. C., Widaman, K. F., Zhang, S., \& Hong, S. (1999). Sample size in factor analysis. Psychological Methods, 4, 84-99.

Marsh, P. J., Langdon, R., Harris, A., \& Coltheart, M. (2013). The case for social-cognitive remediation in schizophrenia: A life well lived is more than remission from psychosis. Australian and New Zealand Journal of Psychiatry, 47, 512-515.

Martin, J. A., \& Penn, D. L. (2002). Attributional style in schizophrenia: An investigation in outpatients with and without persecutory delusions. Schizophrenia Bulletin, 28, 131-141.

Mehta, U. M., Thirthalli, J., Subbakrishna, D. K., Gangadhar, B. N., Eack, S. M., \& Keshavan, M. S. (2013). Social and neuro-cognition as distinct cognitive factors in schizophrenia: A systematic review. Schizophrenia Research, 148, 3-11. 
Melo, S. S., Taylor, J. L., \& Bentall, R. P. (2006). 'Poor me' versus 'bad me' paranoia and the instability of persecutory ideation. Psychology and Psychotherapy: Theory, Research and Practice, 79, 271-287.

Merrin, J., Kinderman, P., \& Bentall, R. P. (2007). 'Jumping to conclusions' and attributional style in persecutory delusions. Cognitive Therapy and Research, 31, 741-758.

Mezulis, A. H., Abramson, L. Y., Hyde, J. S., \& Hankin, B. L. (2004). Is there a universal positivity bias in attributions? A meta-analytic review of individual, developmental, and cultural differences in the self-serving attributional bias. Psychological Bulletin, 130, 711-747.

Mizrahi, R., Addington, J., Remington, G., \& Kapur, S. (2008). Attribution style as a factor in psychosis and symptom resolution. Schizophrenia Research, 104, 220-227.

Moritz, S., Veckenstedt, R., Hottenrott, B., Woodward, T. S., Randjbar, S., \& Lincoln, T. M. (2010). Different sides of the same coin? Intercorrelations of cognitive biases in schizophrenia. Cognitive Neuropsychiatry, 15, 406-421.

Moritz, S., Veckenstedt, R., Randjbar, S., Vitzthum, F., \& Woodward, T. S. (2011). Antipsychotic treatment beyond antipsychotics: Metacognitive intervention for schizophrenia patients improves delusional symptoms. Psychological Medicine, 41, 1823-1832.

Moritz, S., \& Woodward, T. S. (2006). A generalized bias against disconfirmatory evidence in schizophrenia. Psychiatry Research, 142, 157-165.

Moritz, S., Woodward, T. S., \& Burlon, M. (2005). Metacognitive skill training for schizophrenia patients (MCT). Hamburg: VanHam Campus Press. 
Mukherjee, P., Whalley, H. C., McKirdy, J. W., Sprengelmeyer, R., Young, A. W., McIntosh, A. M., .. . Hall, J. (2014). Altered amygdala connectivity within the social brain in schizophrenia. Schizophrenia Bulletin, 40, 152-160.

Murray, G. K. (2011). The emerging biology of delusions. Psychological Medicine, 41, 7-13.

Park, K.-M., Kim, J.-J., Ku, J., Kim, S. Y., Lee, H. R., Kim, S. I., \& Yoon, K.-J. (2009). Neural correlates of attributional style in schizophrenia. Neuroscience Letters, 459, 35-40.

Peterson, C., Semmel, A., von Baeyer, C., Abramson, L. Y., Metalsky, G. I., \& Seligman, M. E. P. (1982). The Attributional Style Questionnaire. Cognitive Therapy and Research, 6, 287-300.

Randall, F., Corcoran, R., Day, J. C., \& Bentall, R. P. (2003). Attenditon, theory of mind, and causal attributions in people with persecutory delusions: A preliminary investigation. Cognitive Neuropsychiatry, 8, 287-294.

Randjbar, S., Veckenstedt, R., Vitzthum, F., Hottenrott, B., \& Moritz, S. (2011). Attributional biases in paranoid schizophrenia: Further evidence for a decreased sense of self-causation in paranoia. Psychosis: Psychological, Social and Integrative Approaches, 3, 74-85.

Reivich, K. (1995). The measurement of explanatory style. In G. M. Buchanan \& M. E. P. Seligman (Eds.), Explanatory style (pp. 21-48). Hillsdale, NJ: Lawrence Erlbaum.

Riccaboni, R., Fresi, F., Bosia, M., Buonocore, M., Leiba, N., Smeraldi, E., \& Cavallaro, R. (2012). Patterns of evidence integration in schizophrenia and delusions. Psychiatry Research, 200, 108-114.

Sanford, N., Lecomte, T., Leclerc, C., Wykes, T., \& Woodward, T. S. (2013). Change in jumping to conclusions linked to change in delusions in early psychosis. Schizophrenia Research, 147, 207-208. 
Sanford, N., Veckenstedt, R., Moritz, S., Balzan, R., \& Woodward, T. S. (2014). Impaired integration of disambiguating evidence in delusional schizophrenia patients. Psychological Medicine, doi: 10.1017/S0033291714000397.

Savla, G. N., Vella, L., Armstrong, C. C., Penn, D. L., \& Twamley, E. W. (2013). Deficits in domains of social cognition in schizophrenia: A meta-analysis of the empirical evidence. Schizophrenia Bulletin, 39, 979-992.

Seidel, E.-M., Satterthwaite, T. D., Eickhoff, S. B., Schneider, F., Gur, R. C., Wolf, D. H., . . . Derntl, B. (2012). Neural correlates of depressive realism - an fMRI study on causal attribution in depression. Journal of Affective Disorders, 138, 268-276.

Sharp, H. M., Fear, C., \& Healy, D. (1997). Attributional style and delusions: An investigation based on delusional content. European Psychiatry, 12, 1-7.

Sheehan, D. V., Lecrubier, Y., Sheehan, K. H., Amorim, P., Janavs, J., Weiller, E., . . Dunbar, G. C. (1998). The Mini-International Neuropsychiatric Interview (MINI): The development and validation of a structured diagnostic psychiatric interview for DSM-IV and ICD-10. The Journal of Clinical Psychiatry, 59, 22-33.

Silverman, R. J., \& Peterson, C. (1993). Explanatory style in schizophrenic and depressed outpatients. Cognitive Therapy and Research, 17, 457-470.

Speechley, W. J., Ngan, E. T.-C., Moritz, S., \& Woodward, T. S. (2012). Impaired evidence integration and delusions in schizophrenia. Journal of Experimental Psychopathology, 3, 688-701.

Speechley, W. J., Whitman, J. C., \& Woodward, T. S. (2010). The contribution of hypersalience to the "jumping to conclusions" bias associated with delusions in schizophrenia. Journal of Psychiatry and Neuroscience, 35, 7-17. 
Speechley, W. J., Woodward, T. S., \& Ngan, E. T. (2013). Failure of conflict to modulate central executive network activity associated with delusions in schizophrenia. Frontiers in Psychiatry, 4, 113.

Stange, J. P., Sylvia, L. G., da Silva Magalhães, P. V., Frank, E., Otto, M. W., Miklowitz, D. J., . .. Deckersbach, T. (2013). Extreme attributions predict transition from depression to mania or hypomania in bipolar disorder. Journal of Psychiatric Research, 47, 1329-1336.

Stange, J. P., Sylvia, L. G., da Silva Magalhães, P. V., Miklowitz, D. J., Otto, M. W., Frank, E., . .. Deckersbach, T. (2013). Extreme attributions predict the course of bipolar depression: Results from the STEP-BD randomized controlled trial of psychosocial treatment. Journal of Clinical Psychiatry, 74, 249-255.

Tabak, N. T., \& Weisman de Mamani, A. G. (2013). Latent profile analysis of healthy schizotypy within the extended psychosis phenotype. Psychiatry Research, 210,10081013.

Tennen, H., \& Herzenberger, S. (1985). Attributional Style Questionnaire. In S. J. Keyser \& R. C. Sweetland (Eds.), Test Critiques. Westport: Test Corporation of America.

Walter, H., Ciaramidaro, A., Adenzato, M., Vasic, N., Ardito, R. B., Erk, S., \& Bara, B. G. (2009). Dysfunction of the social brain in schizophrenia is modulated by intention type: An fMRI study. SCAN, 4, 166-176.

White, P. A. (1991). Ambiguity in the internal/external distinction in causal attribution. Journal of Experimental Social Psychology, 27, 259-270.

Woodward, T. S., Buchy, L., Moritz, S., \& Liotti, M. (2007). A bias against disconfirmatory evidence is associated with delusion proneness in a nonclinical sample. Schizophrenia Bulletin, 33, 1023-1028. 
Woodward, T. S., Moritz, S., \& Chen, E. Y. H. (2006). The contribution of a cognitive bias against disconfirmatory evidence (BADE) to delusions: A study in an Asian sample with first episode schizophrenia spectrum disorders. Schizophrenia Research, 83, 297-298.

Woodward, T. S., Moritz, S., Cuttler, C., \& Whitman, J. (2006). The contribution of a cognitive bias against disconfirmatory evidence (BADE) to delusions in schizophrenia. Journal of Clinical and Experimental Neuropsychology, 28, 605-617.

Woodward, T. S., Moritz, S., Menon, M., \& Klinge, R. (2008). Belief inflexibility in schizophrenia. Cognitive Neuropsychiatry, 13, 267-277.

Woodward, T. S., Munz, M., Leclerc, C., \& Lecomte, T. (2009). Change in delusions is associated with change in "jumping to conclusions". Psychiatry Research, 170, 124-127. 


\section{Appendix A: MATLAB Scripts}

\section{Eigenvalues and Scree Plot (Example from Study 2)}

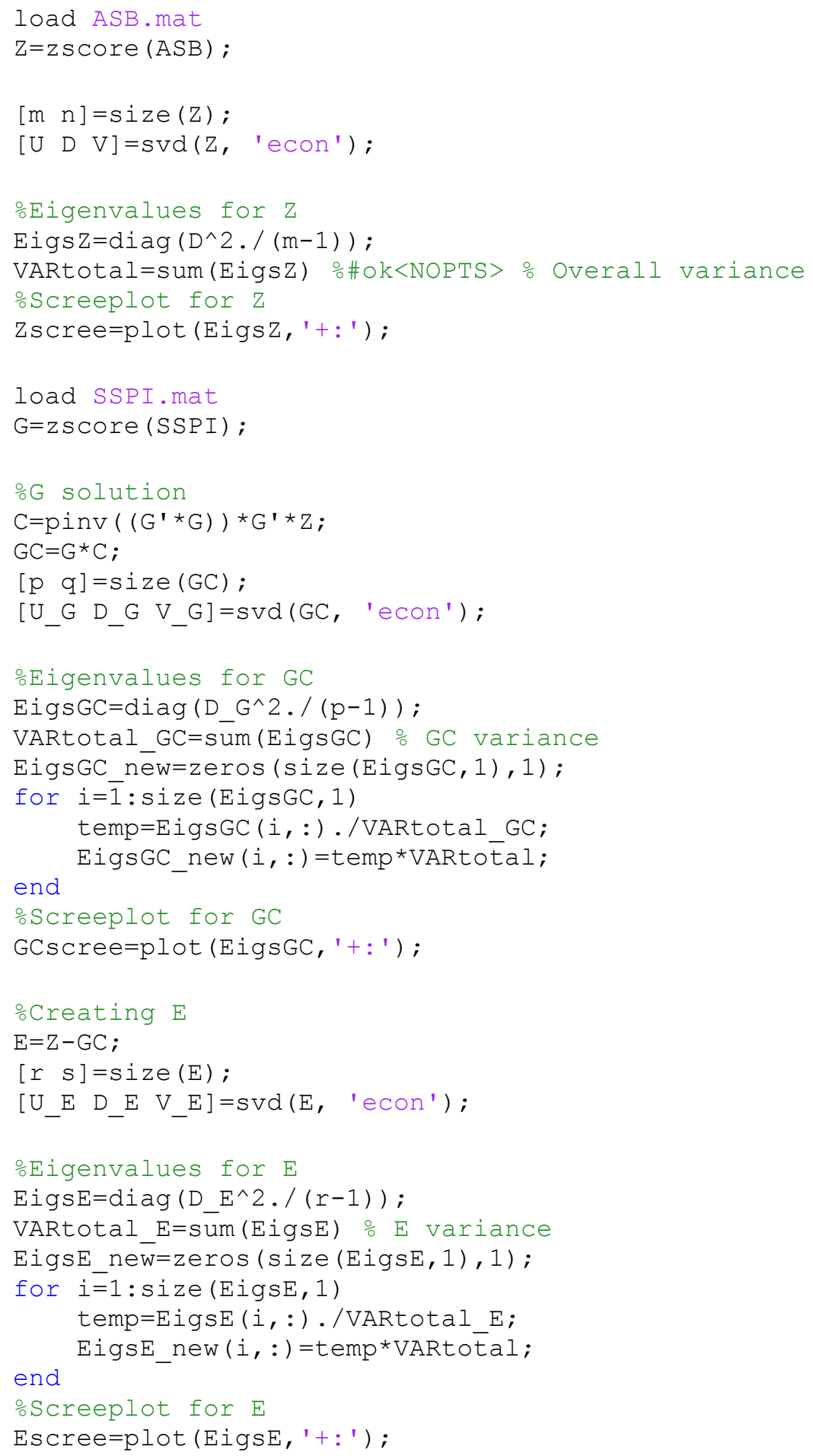




\section{Constrained Principal Component Analysis (Example from Study 2)}

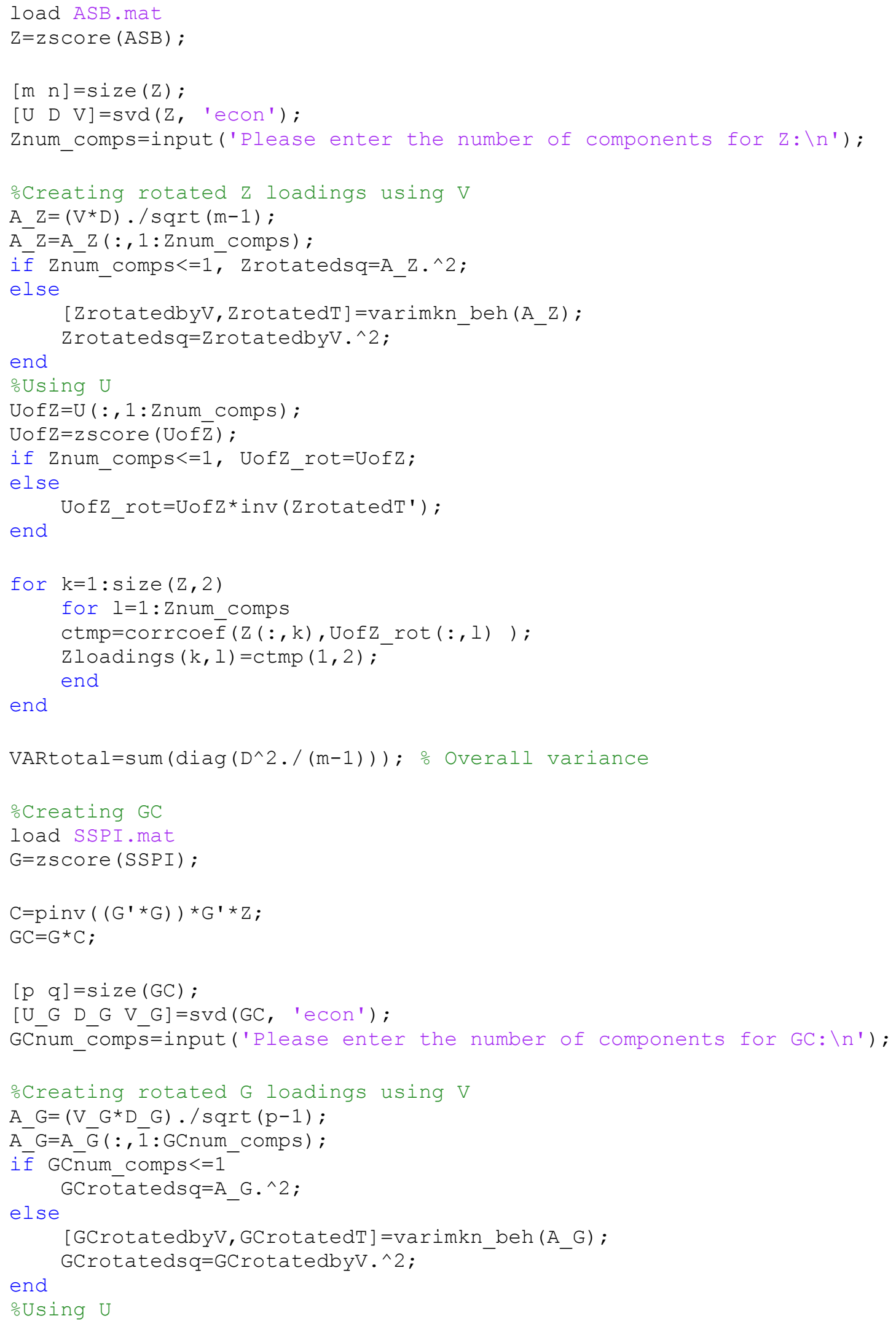




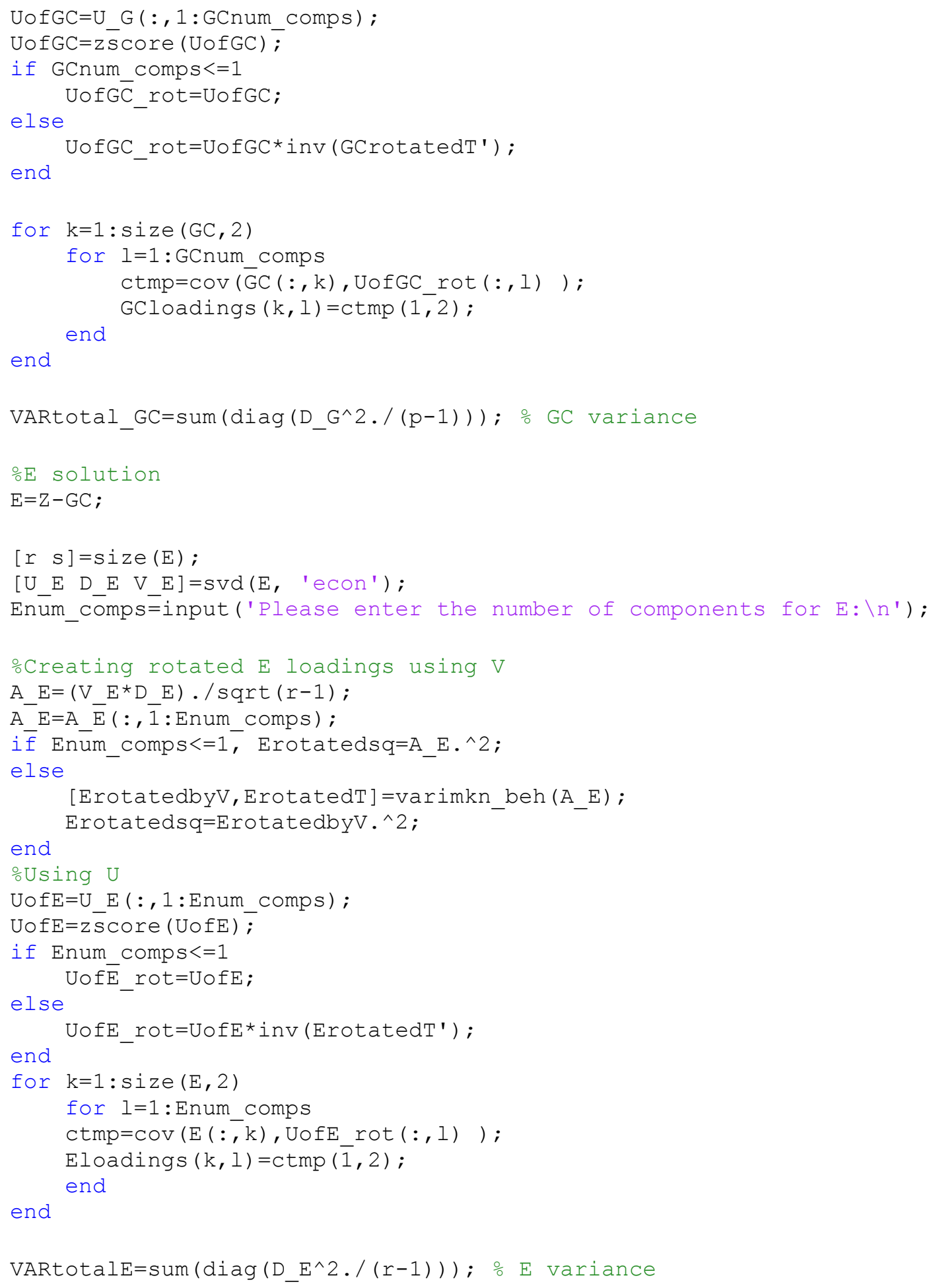




\section{Appendix B: Mathematical Operations}

\section{Principal Component Analysis (PCA)}

PCA as performed in the reported analyses was completed using singular value decomposition (SVD). Given a column-wise mean-centred matrix $X$ of $n$ rows (participants) by $m$ columns (variables), SVD of $X$ is expressed as a factorization of the form:

$$
{ }_{n} X_{m}={ }_{n} U_{n} X_{n} D_{m} \times{ }_{m} V_{m}^{\mathrm{T}}
$$

where ${ }^{\mathrm{T}}$ indicates that the preceding matrix is transposed, $U$ contains the left-singular vectors of $X$ (or the eigenvectors of the $n$ by $n$ covariance matrix $X X^{\mathrm{T}}$ ), $D$ is a rectangular diagonal matrix whose elements (the singular values of $X$ ) are the square roots of the eigenvalues $(\lambda)$ of the corresponding eigenvectors in $U$ (such that $\sum \lambda=\mathrm{SS}_{X}$, where SS is the sum of squares), and $V$ (the right-singular vectors of $X$ ) is a loading matrix that serves to transform the variables to and from component space.

The percentage of variance in $X$ that each component accounts for can be calculated from the following:

$$
\operatorname{VarC}_{\mathrm{i}}=\left(\frac{\lambda_{\mathrm{i}}}{\sum \lambda \mathrm{r}}\right) /(n-1)
$$

The component loadings, which indicate the degrees to which each of the variables contribute to each component, are calculated as follows:

$$
\text { CompLoadings }=(V \times D) / \sqrt{ }(n-1)
$$

which is equivalent to the correlation between $U$ (from equation 1) and $X$. 
The component weights, which are the coefficients applied to each standardized variable to compute the component score as a weighted aggregate, are calculated as follows:

$$
\text { CompWeights }=A\left(A^{\mathrm{T}} \times A\right)^{-1}
$$

where $A=$ the matrix of component loadings.

\section{Constrained Principal Component Analysis (CPCA)}

As described in Section 2.3.2., the external analysis of a CPCA model involving a single set of independent variables is expressed as follows:

$$
{ }_{n} Z_{m}=\left({ }_{n} G_{v} \times{ }_{v} C_{m}\right)+{ }_{n} E_{m}
$$

where $Z$ is the dependent variable (criterion), $G$ is a set of independent variables (predictor), $E$ is a matrix of residuals, and

$$
C=\left(G^{\mathrm{T}} \times G\right)^{-1} \times G^{\mathrm{T}} \times Z
$$

is the set of regression coefficients (i.e., betas) that are applied to $G$ to produce the predicted scores $(G C)$.

$Z$ consists of participants' scores on a set of dependent variables, with one row per participant and one column per variable, producing a matrix of $n$ participants by $m$ dependent variables. $G$ consists of the independent variables that are expected to relate to $Z$ (e.g., indicator variables coding group membership for between-groups analyses, or participants' scores on a symptom scale), forming a matrix of $n$ participants by $v$ independent variables. The external analysis, then, amounts to a multivariate multiple regression, dividing $Z$ into the portion of variance that can be predicted by $G(G C)$ and that which cannot be predicted by $G(E)$. 
The internal analysis involves performing PCA on each of the sources of variance resulting from the external analysis. The component solutions that emerge (total, predicted, and residual solutions) can then be examined to determine which components in $Z$ are predictable from the independent variables $(G)$ and which are not. To determine the degree to which each of the independent variables contributes to the components obtained from the predicted solution $(G C)$, correlations are computed between participants' scores on each component ( $U$ from equation 1) and the independent variables in $G$ to produce the set of predictor loadings (termed " $G$ loadings" in the reported analyses).

\section{Generalized Structured Component Analysis (GSCA)}

In GSCA, a specified model can be expressed as:

$$
\begin{gathered}
Z V=Z W A+E \\
\Psi=\Gamma A+E
\end{gathered}
$$

where $Z$ is an $n$ (participants) by $j$ (observed variables) matrix of normalized observed variables (e.g., attribution ratings on the ASB task), $V$ is a $j$ by $t$ matrix of component weights associated with the endogenous variables (i.e., components that are influenced by other components in the model, or dependent variables), $W$ is a $j$ by $d$ matrix of component weights for the exogenous variables (i.e., components that are not influenced by other components in the model, or independent variables), $A$ is a $d$ by $t$ supermatrix consisting of a matrix of component loadings (C) and a matrix of path coefficients between components $(B)$ such that $A=[C, B]$, and $E$ is an $n$ by $t$ matrix of residuals. $\Psi$, then, is an $n$ by $t$ matrix of all endogenous observed and latent variables, and $\Gamma$ is an $n$ by $d$ matrix that contains the latent variable (i.e., component) scores for each participant. 
The unknown parameters $V, W$, and $A$ are estimated such that the sum of squares of the residuals, $E=Z V-Z W A=\Psi-\Gamma A$, is as small as possible. This involves minimizing

$$
\begin{aligned}
f & =\mathrm{SS}(Z V-Z W A) \\
& =\mathrm{SS}(\Psi-\Gamma A)
\end{aligned}
$$

with respect to $V, W$, and $A$, where $\operatorname{SS}(X)=$ the sum of the diagonal entries of $\left(X^{\mathrm{T}} X\right)$.

The overall fit of a hypothesized model is calculated from the proportion of variance of all the endogenous observed and latent variables that is explained by the model, given by

$$
\mathrm{FIT}=1-\frac{\mathrm{SS}(\Psi-\Gamma A)}{\mathrm{SS}(\Psi)}
$$

The larger the fit value (which ranges from 0 to 1 ), the more variance is explained by the model. Thus, the relationship between the model and the observed data is summarized as a function of the sum of squares of the residuals.

Multi-group GSCA fits the same model (7) to multiple groups of participants simultaneously. Given $L$ groups, this can be expressed as

$$
\begin{aligned}
{\left[\begin{array}{c}
\Psi_{1} \\
\vdots \\
\Psi_{L}
\end{array}\right] } & =\left[\begin{array}{lll}
\Gamma_{1} & & 0 \\
& \ddots & \\
0 & & \Gamma_{L}
\end{array}\right]\left[\begin{array}{c}
A_{1} \\
\vdots \\
A_{L}
\end{array}\right]+\left[\begin{array}{c}
E_{1} \\
\vdots \\
E_{L}
\end{array}\right], \\
\widetilde{\Psi} & =\widetilde{\Gamma} \tilde{A}+\tilde{E} .
\end{aligned}
$$

$L$ sets of parameters can be considered as a single set of parameters as in a single group of participants, so that the same optimization procedure used for model (7) may be used for model (10). 